\title{
A Numerical Investigation of Dual-Rotor Horizontal Axis Wind Turbines using an In-House Vortex Filament Code (DR_HAWT)
}

by

Kenny Lee Slew, B.Eng.

A thesis submitted to the Faculty of Graduate and Postdoctoral Affairs in partial fulfillment of the requirements for the degree of

Master of Applied Science

in

Mechanical Engineering

Ottawa-Carleton Institute for Mechanical and Aerospace Engineering

Department of Mechanical and Aerospace Engineering

Carleton University

Ottawa, Ontario

January, 2017

(C) Copyright

Kenny Lee Slew, 2017 


\section{Abstract}

A numerical study was carried out using an in-house code named DR_HAWT (DualRotor Horizontal-Axis Wind Turbine code), to identify non-dimensional parameters for dual-rotor wind turbines (DRWTs). DR_HAWT, which was implemented by the current author to predict the performance of single and dual-rotor wind turbines, uses a free vortex wake method with vortex filaments to represent the rotor and its wake. This vortex code was verified and validated using various blade-vortex interaction case studies and two well-known wind tunnel experiments (NREL Phase VI and MEXICO wind turbines).

Based on some important DRWT parameters such as the rotor speeds, rotor diameters and the separation distance between the rotors, three dimensionless parameters were derived from the Buckingham Pi theorem. These three parameters define the ratio of diameters (diameter ratio, DR), the ratio of the separation distance and the downwind diameter (gap ratio, GR), and the ratio of the product of the upwind angular velocity and the downwind rotor radius and the oncoming wind velocity (combined

tip-speed ratio, CTSR). In addition, the power output of each DRWT was normalized with the total power generated by equivalent single-rotor turbines.

Hypothetical DRWT models were created using geometrically-scaled NREL Phase VI rotor geometry and operating conditions in order to confirm the validity of these 
parameters. The variation in normalized output power as a function of the dimensionless parameters suggests that an improved performance of DRWTs can be obtained at lower diameter and gap ratios. The study also revealed that the effect of having an auxiliary upwind rotor reduces the angle of attack along the inboard section of the downwind blade. Finally, the NREL Phase VI rotor equipped with a $5 \mathrm{~m}$ geometrically-scaled upwind rotor was found to generate more than the combined power output of two equivalent single-rotors at 7.5 CTSR. It was hypothesized that this extra power results from a shift in the operating range of the blade due to the variation in angle of attack. 


\section{Acknowledgments}

First and foremost, I am very grateful to my supervisor, Dr. Edgar Matida, for giving me the opportunity to pursue graduate studies. Thank you for believing in me and providing support, guidance, and encouragement throughout these few years.

I would also like to thank Amin Fereidooni, John O'Keefe and ZEC Wind Power Corp., specially Georges El-Hage, Patrick Tawagi, and Michael Hou, for their collaboration during the initial stage of this thesis. As well as Neil McFadyen for his help and technical support with the computer resources on campus. I am also truly grateful to Dr. Scott Schreck from the National Renewable Energy Laboratory (NREL) and Dr. Gerard Schepers from the Energy Research Centre of the Netherlands (ECN) for supplying the measurement data of the NREL Phase VI and MEXICO experiments, respectively.

Many thanks go out to my friends and colleagues for providing well-needed distractions and for listening to my frequent rants. Special thanks to Michael Miller for his advice and discussions in times of trouble as well as for providing a fresh pair of eyes when troubleshooting the code. Last but not least, I would like to thank my family for their encouragement and support. 


\section{Table of Contents}

$\begin{array}{lll}\text { Abstract } & \text { ii }\end{array}$

Acknowledgments $\quad$ iv

Table of Contents $\quad$ v

List of Tables $\quad$ viii

List of Figures $\quad$ ix

Nomenclature $\quad$ xiv

1 Introduction 1

1.1 Principles of Wind Turbines $\ldots \ldots \ldots \ldots \ldots$

1.2 Dual-Rotor Wind Turbines . . . . . . . . . . . . . . . 6

1.3 Motivation . . . . . . . . . . . . . . . . . 9

1.4 Structure of Thesis . . . . . . . . . . . . . . . . 10

2 Review of Performance Prediction Methods 11

2.1 Wind Tunnel Experiments . . . . . . . . . . . . . . . . . . . . 12

2.1.1 NREL Phase VI Wind Turbine . . . . . . . . . . . . . 13

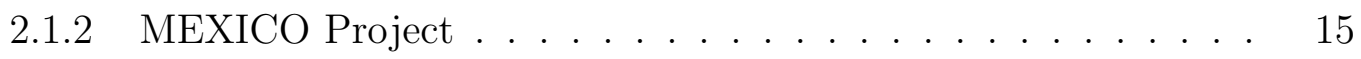

2.2 Blade Element Momentum (BEM) Theory . . . . . . . . . . . 16 
2.3 Vortex Methods . . . . . . . . . . . . . . . . . . . . . . . . 19

2.4 CFD Models . . . . . . . . . . . . . . . . . . . . 20

2.5 Comparison of Prediction Tools . . . . . . . . . . . . . . . . 21

3 DR_HAWT: Vortex Filament Code 23

3.1 Governing Equations . . . . . . . . . . . . . . . . 24

3.2 Coordinate System . . . . . . . . . . . . . . . . 25

3.3 Element Bound Vorticity . . . . . . . . . . . . . . . 28

3.4 Vortex Shedding . . . . . . . . . . . . . . . 30

3.5 Wake Convection ... . . . . . . . . . . . . . 33

3.6 Induced Velocity . . . . . . . . . . . . . . . . . 34

3.7 Stall Delay Model . . . . . . . . . . . . . . . . . . . . . . . 39

3.8 Blade loading and Rotor Performance . . . . . . . . . . . . . . . . . . 42

3.9 Parallel Implementation . . . . . . . . . . . . . . . . . . . 45

3.10 Program Structure . . . . . . . . . . . . . . . . . . . . . 47

4 Verification and Validation Methodology $\quad 51$

4.1 Elliptic Wing . . . . . . . . . . . . . . . . 51

4.2 Blade-Vortex Interaction . . . . . . . . . . . . . . . . . 54

4.3 NREL Phase VI Wind Turbine . . . . . . . . . . . . . . . 57

4.3.1 Convergence Study . . . . . . . . . . . . . . . . . 57

4.3.2 Comparison with Experimental Data . . . . . . . . . . . 62

4.4 MEXICO Wind Turbine . . . . . . . . . . . . 66

4.5 Dual-Rotor Validation . . . . . . . . . . . . . . . . . 68

5 Study Approach and Results $\quad 72$

5.1 Dimensional Analysis . . . . . . . . . . . . . . . . . . . 72 
5.2 Parametric Sweep . . . . . . . . . . . . . . . 76

5.3 Results and Discussion . . . . . . . . . . . . . . . . 77

5.3.1 CASE D01: Effect of Gap Ratio vs CTSR . . . . . . . . . 77

5.3.2 CASE D02: Effect of Diameter Ratio vs CTSR . . . . . . . 81

5.3.3 CASE D03: Effect of Diameter Ratio vs Gap Ratio . . . . . . 86

5.3.4 CASE D04: Scaling Effect . . . . . . . . . . . 89

6 Conclusions and Recommendations $\quad 91$

6.1 Prediction Tool . . . . . . . . . . . . . . . . . . . . . . . . . 91

6.2 Dual-Rotor Study . . . . . . . . . . . . . . . . . . 93

$\begin{array}{ll}\text { List of References } & 106\end{array}$

$\begin{array}{ll}\text { Appendix A Full-Cosine Discretization } & 107\end{array}$

$\begin{array}{lll}\text { Appendix B Inverse BEM } & 108\end{array}$ 


\section{List of Tables}

2.1 Summary of performance prediction software for wind turbines . . . . 12

3.1 Summary of functions used in stall delay models . . . . . . . . . . . . 41

4.1 Description of the NREL Phase VI wind turbine geometry provided in Ref. $[47] \ldots \ldots \ldots \ldots \ldots$. . . . . . . . . . . . . . . . . . . .

5.1 Summary of dependent and independent variables for DRWT . . . . . 74

5.2 Summary of DRWT parametric study . . . . . . . . . . . . . . . 76 


\section{List of Figures}

1.1 Wind power generation in Canada over the past 16 years . . . . . . 2

1.2 Schematic of a typical (a) Vertical Axis Wind Turbine (VAWT) and (b) Horizontal Axis Wind Turbine (HAWT) . . . . . . . . . . . . . 3

1.3 Aerodynamic loads acting on a section of a wind turbine blade, twisted at an angle $\beta \ldots \ldots \ldots \ldots$. . . . . . . . . . . . . 4

1.4 Schematic of the two main type of dual-rotor wind turbines . . . . . . 6

1.5 Schematic of the velocity triangles for co- (left) and counter-rotating (right) wind turbines . . . . . . . . . . . . . . . . . 7

2.1 NREL Phase VI turbine mounted in NASA Ames $24.4 \mathrm{~m} \times 36.6 \mathrm{~m}$ wind tunnel (left) [49] and MEXICO turbine mounted in DNW $9.5 \mathrm{~m}$

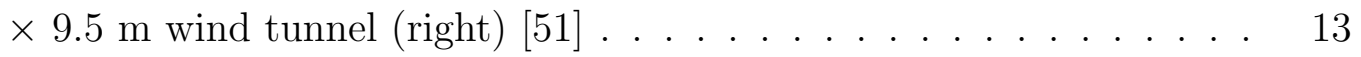

2.2 NREL Phase VI blade geometry . . . . . . . . . . . . . . . . . . . 14

2.3 NREL Phase VI blind comparison results extracted from Ref. [48]. Experimental data provided by Dr. Schreck [52] . . . . . . . . . 15

2.4 MEXICO blade geometry . . . . . . . . . . . . . . 16

2.5 MEXICO torque measurements compared with the prediction from various codes (extracted from Ref. [51]). Experimental data provided by Dr. Schepers $[55]$. . . . . . . . . . . . . . . . . . . 17 
2.6 MEXICO axial thrust measurements compared with the prediction from various codes (extracted from Ref. [51]). Experimental data provided by Dr. Schepers [55] . . . . . . . . . . . . . . . . . . . 17

2.7 Power prediction of the NREL Phase VI wind turbine using the QBlade, DR_HAWT, and Ellipsys3D codes . . . . . . . . . . . 22

3.1 Definition of circulation . . . . . . . . . . . . . 25

$3.23 \mathrm{D}$ model representing wind turbine in DR_HAWT . . . . . . . . . 26

3.3 Velocity components acting on blade element . . . . . . . . . . . 29

3.4 Bound vortex calculation using the predictor corrector method . . . . 31

3.5 Schematic of the bound (Green), spanwise (Red) and trailing (Blue) vortices representing the wake behind a blade consisting of two elements after several timesteps. Adapted from Strickland et al. [41]. . .

3.6 Velocity induced by a straight filament vortex of strength $\Gamma_{v}$ with vortex core representation . . . . . . . . . . . . . . 35

3.7 Induced velocity of a filament vortex as a function of dimensionless

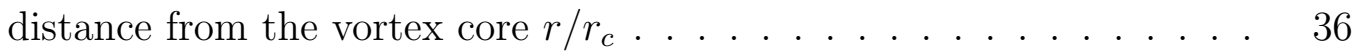

3.8 Axial induced velocity vortex ring comparison as a function of relative core size $r_{c} / R \ldots \ldots \ldots \ldots \ldots \ldots \ldots$

3.9 Effect of various 3D corrections applied to the wind tunnel (2D) data from OSU [47] for the S809 airfoil located at 46.6\% $\mathrm{R}$ along a blade rotating at $7.5 \mathrm{rad} / \mathrm{s}$ and $R e=6.5 \times 10^{5} \ldots \ldots$. . . . . . . . . 42

3.10 Load coefficients acting on blade element . . . . . . . . . . . . 43

3.11 Parallel capability of DR_HAWT . . . . . . . . . . . . 46

3.12 Verification of parallel implementation in DR_HAWT . . . . . . . . 46

3.13 Program structure . . . . . . . . . . . . . . . . . . 48 
3.14 NREL Phase VI Wake visualization using ParaView where the wake from each blade is illustrated with a different color . . . . . . . . . 50

4.1 Chord and spanwise circulation distributions for a typical elliptical wing with low aspect ratio . . . . . . . . . . . . 52

4.2 Comparison between computed and analytical downwash distribution over an elliptic wing with high aspect ratio . . . . . . . . . . . 54

4.3 Schematic of a blade-vortex interaction phenomenon . . . . . . . . . 55

4.4 Induced velocity variation of a stationary blade element as a vortex with prescribed strength approaches. The approximate location of the stationary blade element is also shown . . . . . . . . . . . 56

4.5 Coefficient of power as a function of the variation in (a) azimuth angle increment, (b) vortex core radius, (c) wake length, and (d) number of discretized sections . . . . . . . . . . . . . . . . 60

4.6 Performance prediction comparison with NREL experimental data: (a) Torque (Top) and (b) Thrust (Bottom) . . . . . . . . . . . . 63

4.7 Comparison between computed and measured angle of attack distribution over the span of the NREL Phase VI blade . . . . . . . . . . . . 65

4.8 MEXICO blade chord length and twist distribution . . . . . . . . 67

4.9 Performance prediction comparison with MEXICO experimental data: (a) Torque (Top) and (b) axial thrust (Bottom) . . . . . . . . . . 69

4.10 Comparison between computed and measured angle of attack distribution over the span of the MEXICO blade . . . . . . . . . . . 70

5.1 Schematic of the dual-rotor horizontal axis wind turbine's performance parameters used in the present study . . . . . . . . . . . . 73

5.2 Schematic of the four DRWT models investigated in Case D01 . . . . 78 
5.3 Power output comparison between four DRWT having diameter ratio of 0.50 at various gap ratios $(\mathrm{GR}=0.25,0.50,0.75$, and 1.00$) \quad \ldots \quad 79$

5.4 Wake visualization of a half-scaled NREL Phase VI rotor generated from DR_HAWT . . . . . . . . . . . . . . . . . . 79

5.5 Performance of the upwind (solid lines) and downwind (dashed lines) rotors compared to their respective SRWT as a function of gap ratio . 80

5.6 S809 lift curve at $\mathrm{Re}=360,000 \ldots \ldots$. . . . . . . . . . . . 81

5.7 Schematic of the four DRWT models investigated in Case D02 . . . . 82

5.8 Power output comparison between four DRWT having various diameter ratios $(\mathrm{DR}=0.25,0.50,0.75$, and 1.00$)$ and a gap ratio of 0.50 as a function of combined tip-speed ratio . . . . . . . . . . . .

5.9 Performance of the upwind (solid lines) and downwind (dashed lines) rotors compared to their respective SRWT as a function of combined tip-speed ratio . . . . . . . . . . . . . . .

5.10 Power output comparison between four DRWT having various diameter ratios $(\mathrm{DR}=0.25,0.50,0.75$, and 1.00$)$ and a gap ratio of 0.50 as a function of freestream velocity . . . . . . . . . . . . . .

5.11 Angle of attack comparison between the downwind rotor of DRWT with diameter ratios ranging from 0.25 to 1.00 and its equivalent SRWT at a freestream velocity of $10.0 \mathrm{~m} / \mathrm{s} \ldots \ldots \ldots$

5.12 Schematic of the 16 DRWT models investigated in Case D03 . . . . . 87

5.13 Power output comparison between four DRWT having various diameter ratios $(\mathrm{DR}=0.25,0.50,0.75$, and 1.00$)$ at a CTSR of $7.5 \ldots$.

5.14 Power output comparison between four DRWT having various diameter ratios $(\mathrm{DR}=0.25,0.50,0.75$, and 1.00$)$ at $10 \mathrm{~m} / \mathrm{s}$ freestream velocity 
5.15 Performance of the upwind (solid lines) and downwind (dashed lines) rotors compared to their respective SRWT as a function of gap ratio at $10 \mathrm{~m} / \mathrm{s}$ freestream velocity $\ldots \ldots \ldots$. . . . . . . . . 89

5.16 Schematic of the three DRWT models investigated in Case D04 . . . 90

5.17 Power output comparison between three geometrically-scaled DRWT with diameter and gap ratios of 0.5 (Dashed lines represent the constant Reynolds number case) . . . . . . . . . . . . . . . . . . . . . 90

A.1 Full-cosine discretization method applied on the NREL Phase VI . . 107 


\section{Nomenclature}

\section{Latin Letters}

A Swept area of wind turbine rotor

$\left[m^{2}\right]$

R

Aspect ratio

$[-]$

$B N \quad$ Blade number

$[-]$

$c$

Chord length

$[m]$

$C_{D}$

Coefficient of drag

$[-]$

$C_{L} \quad$ Coefficient of lift

$[-]$

$C_{n}$

Coefficient of normal force

$[N]$

$C_{P}$

Coefficient of power

$[-]$

$C_{Q}$

Coefficient of torque

$[-]$

$C_{T}$

Coefficient of thrust

$[-]$

$C_{t} \quad$ Coefficient of tangential force

$D$

Drag force

$[N]$

$D, D_{1}, D_{2} \quad$ Rotor diameter

$[m]$ 


\begin{tabular}{|c|c|c|}
\hline$F_{n}$ & Normal force & {$[N]$} \\
\hline$F_{t}$ & Tangential force & {$[N]$} \\
\hline$L$ & Lift force & {$[N]$} \\
\hline$L$ & Separation distance between rotors & {$[m]$} \\
\hline$N B$ & Number of blades & {$[-]$} \\
\hline$N T$ & Timestep & {$[-]$} \\
\hline$P_{\text {dual }}$ & Power generated by dual-rotor wind turbine & {$[W]$} \\
\hline$P_{\max }$ & Maximum available power & {$[W]$} \\
\hline$P_{\text {norm }}$ & Normalized power & {$[-]$} \\
\hline \multirow[t]{2}{*}{$P_{\text {singles }}$} & Sum of power generated by two single-rotor & {$[W]$} \\
\hline & wind turbines & \\
\hline$Q$ & Rotor torque & {$[N m]$} \\
\hline$R$ & Radius of wind turbine rotor & {$[m]$} \\
\hline$r$ & Radial distance from the hub center & {$[m]$} \\
\hline$r_{c}$ & Vortex core radius & {$[m]$} \\
\hline$R e$ & Reynolds number & {$[-]$} \\
\hline$r_{o}$ & Vortex core radius at $t=0$ & {$[m]$} \\
\hline $\mathbf{s}, \mathbf{n}, \mathbf{c}$ & Unit vectors defining the blade element & {$[-]$} \\
\hline
\end{tabular}


$U_{t}, V_{t}, W_{t} \quad$ Magnitude of induced velocity in $x, y$, and $z \quad[m / s]$

directions at time $t$

$\mathbf{V}_{\text {ind }}$

$V_{o}$

$\mathbf{V}_{o}$

$\mathbf{V}_{\text {rel }}$

$\mathbf{V}_{T}$

$x, y, z$
Induced velocity vector

Magnitude of the oncoming wind velocity

Wind velocity vector

Relative velocity vector

Blade velocity vector

Global coordinates of the aerodynamic centre $[\mathrm{m} / \mathrm{s}]$

$[\mathrm{m} / \mathrm{s}]$

$[\mathrm{m} / \mathrm{s}]$

$[\mathrm{m} / \mathrm{s}]$

$[\mathrm{m} / \mathrm{s}]$

$[m]$ 


\section{Greek Letters}

\begin{tabular}{|c|c|c|}
\hline$\alpha$ & Angle of attack & {$\left[{ }^{\circ}\right]$} \\
\hline$\beta$ & Sum of twist and pitch angles & {$[0]$} \\
\hline$\Gamma_{B}$ & Bound vortex strength & {$\left[m^{2} / s\right]$} \\
\hline$\Gamma_{S}$ & Spanwise vortex strength & {$\left[m^{2} / s\right]$} \\
\hline$\Gamma_{T}$ & Trailing vortex strength & {$\left[m^{2} / s\right]$} \\
\hline$\delta$ & Eddy viscosity coefficient & {$[-]$} \\
\hline$\delta_{c}$ & Cut-off radius parameter & {$[\%]$} \\
\hline$\Delta t$ & Timestep increment & {$[s]$} \\
\hline$\zeta$ & Vorticity & {$\left[s^{-1}\right]$} \\
\hline$\theta$ & Angle Rotated by Blade 1 & {$\left[{ }^{\circ}\right]$} \\
\hline$\theta_{B}$ & Blade Azimuth Angle & {$\left[{ }^{0}\right]$} \\
\hline$\lambda$ & Tip-speed ratio & {$[-]$} \\
\hline$\mu$ & Fluid Viscosity & {$[\mathrm{kg} / \mathrm{ms}]$} \\
\hline$\nu$ & Fluid Kinemetic Viscosity & {$\left[m^{2} / s\right]$} \\
\hline$\rho$ & Fluid density & {$\left[\mathrm{kg} / \mathrm{m}^{3}\right]$} \\
\hline$\phi$ & Flow angle $(\phi=\alpha+\beta)$ & {$[0]$} \\
\hline$\omega, \omega_{1}, \omega_{2}$ & Rotor angular velocity & {$[\mathrm{rad} / \mathrm{s}]$} \\
\hline
\end{tabular}




\begin{tabular}{|c|c|}
\hline $\mathrm{AC}$ & Aerodynamic center \\
\hline $\mathrm{AOA}$ & Angle of attack \\
\hline BEM & Blade element momentum theory \\
\hline BET & Blade element theory \\
\hline $\mathrm{CFD}$ & Computational fluid dynamics \\
\hline CTSR & Combined tip-speed ratio \\
\hline DES & Detached eddy simulation \\
\hline $\mathrm{DR}$ & Diameter ratio \\
\hline DR_HAWT & Dual-rotor horizontal axis wind turbine code \\
\hline DRWT & Dual-rotor wind turbine \\
\hline FWM & Free-wake method \\
\hline GCS & Global coordinate system \\
\hline GR & Gap ratio \\
\hline HAWT & Horizontal axis wind turbine \\
\hline LCS & Local coordinate system \\
\hline LES & Large eddy simulation \\
\hline LFA & Local flow angle \\
\hline
\end{tabular}




$\begin{array}{ll}\text { LLT } & \text { Lifting-line theory } \\ \text { MEXICO } & \text { Model experiments in controlled conditions } \\ \text { NREL } & \text { National renewable energy laboratory } \\ \text { PWM } & \text { Prescribed-wake method } \\ \text { RANS } & \text { Reynolds averaged Navier-Stokes } \\ \text { SRWT } & \text { Single-rotor wind turbine } \\ \text { TSR } & \text { Tip-speed ratio } \\ \text { UAE } & \text { Unsteady aerodynamic experiments } \\ \text { VAWT } & \text { Vertical axis wind turbine }\end{array}$




\section{Chapter 1}

\section{Introduction}

With the depletion of petroleum resources as well as the increasing environmental issues, viz. climate change, renewable energy such as hydro, wind, and solar, are potential replacements for fossil-fuel-based energy as a source of electricity generation. In 2014, Ontario became the first North American jurisdiction to replace coal with wind and solar energies as a source of power generation [1]. Moreover, in 2008, the Canadian Wind Energy Association (CanWEA) proposed a long term goal of produce $20 \%$ of Canada's electricity from wind by 2025 [2]. Since then, wind energy has rapidly expanded, as can be seen in Figure 1.1, with over 11,000 MW of electricity generated by wind turbines at the end of 2015, which is equivalent to approximately $5 \%$ of Canada's demand in electrical power [3].

Over the past decades, greater emphases have been directed to improve the efficiency of single-rotor wind turbines (SRWTs) by increasing their swept area (i.e., the area of the circle produced by rotating blades) which allows for more power to be extracted since the power output scales with the rotor diameter squared [4]. Other examples to increase wind turbines' efficiency are to optimize the layout of wind farms, and install vortex generators on turbine blades. In more recent years, a growing interest in dual-rotor systems which have been extensively employed in the 


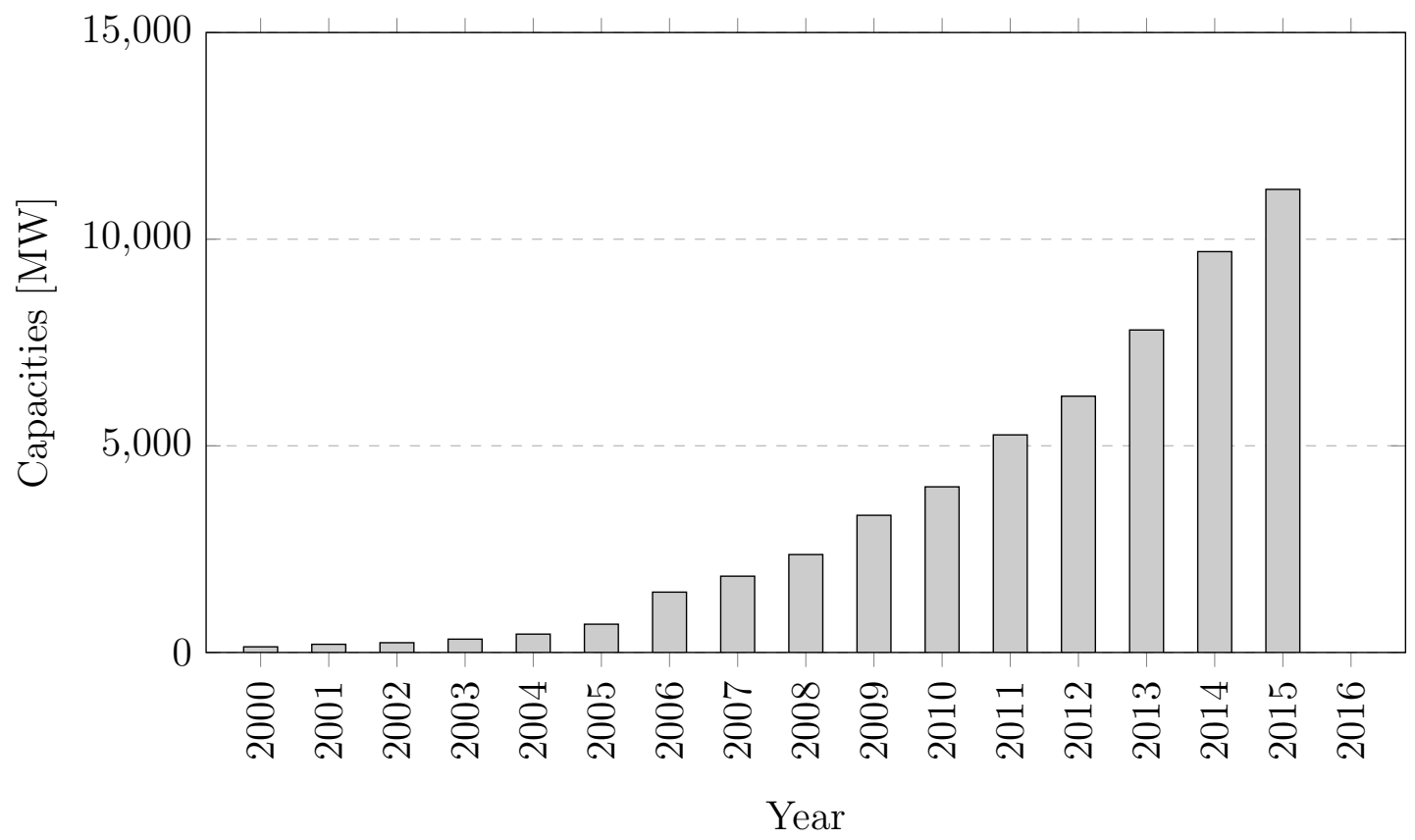

Figure 1.1: Wind power generation in Canada over the past 16 years [3]

aerospace (e.g., the Soviet Ka-32 helicopter) and marine (e.g., the Mark 46 torpedo with contra rotating propellers) applications - to increase the aerodynamic efficiency of the systems while eliminating the asymmetrical torque experienced by conventional single-rotors - have resurfaced. Although the idea of increasing the amount of rotors per tower is not well accepted within the wind industry, university researchers from the University of Ottawa [5] and the Iowa State University (ISU) [6], to name a few, are investigating the potential of Dual-Rotor Wind Turbine (DRWT) systems due to its ability to extract more energy per tower. In addition, dual-rotor systems have the potential to lower the overall cost by reducing the number of towers which accounts for around $14 \%-17 \%$ of the total cost of the turbine including operation and management, grid connection, and foundation costs [7-9], albeit the need for stronger tower and more complex gearbox. 
This chapter describes the fundamental of conventional wind turbines (horizontal and vertical axis wind turbines) and provides a literature survey on recent DRWT studies. Finally, the motivation behind this work and the structure of the thesis will also be outlined.

\subsection{Principles of Wind Turbines}

Wind turbines are rotary machines that extract the kinetic energy of the wind and convert it to electricity via generators. There are two main types of wind turbines, namely Vertical Axis Wind Turbine (VAWT) and Horizontal Axis Wind Turbine (HAWT), as shown in Figure 1.2. As its name implies, VAWT which can either be liftbased or drag-based turbine, rotates vertically with its rotational axis perpendicular
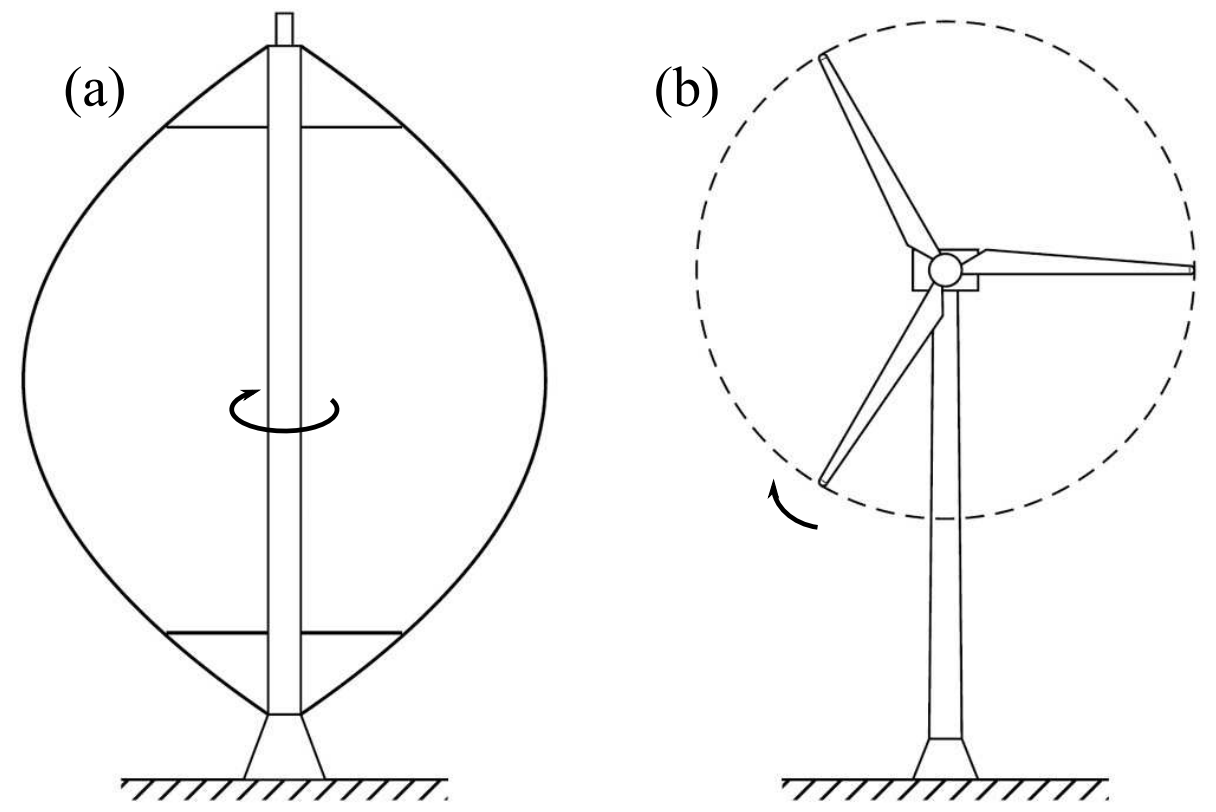

Figure 1.2: Schematic of a typical (a) Vertical Axis Wind Turbine (VAWT) and (b) Horizontal Axis Wind Turbine (HAWT) 
to the incoming wind to generate electricity. HAWT, on the other hand, is a liftbased turbine which uses a propeller-type rotor with its rotational axis parallel to the incoming wind. Since the current work focuses on HAWTs, the term wind turbine will be used to refer to the horizontal axis configuration throughout this thesis.

The blade of a wind turbine is shaped, from a thick cylindrical section at the root to a thin airfoil section at the tip, to achieve the best equilibrium between its aerodynamic and structural properties. In addition to having various cross-sectional shapes, the blade is twisted to take advantage of a more ideal angle of attack $(\alpha)$ - the angle of the blade relative to the apparent wind - along the blade span. As the blade rotates, aerodynamic loads are generated and converted to mechanical power through the turbine shaft. These loads comprise of the lift $(L)$ and drag $(D)$ forces and can be resolved into normal $\left(F_{n}\right)$ and tangential $\left(F_{t}\right)$ forces, acting perpendicular and parallel to the rotor plane, respectively. Figure 1.3 illustrates the aerodynamic loads experienced on an airfoil section of the blade.

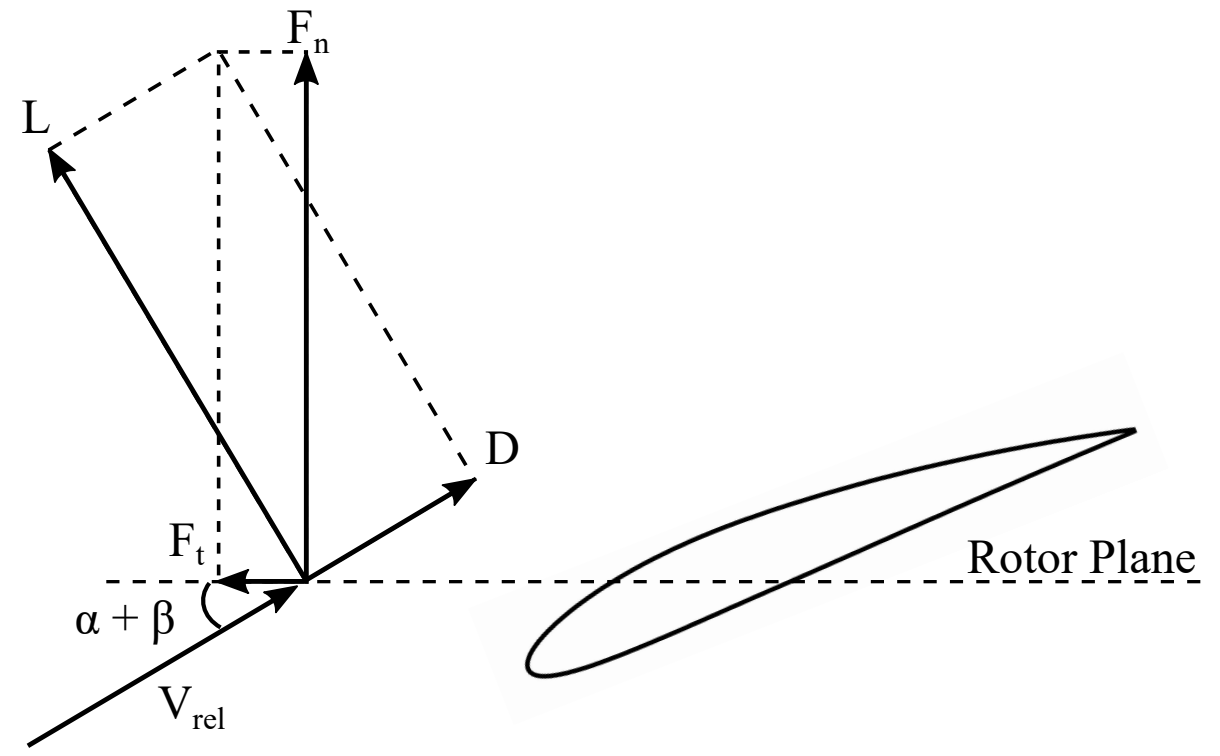

Figure 1.3: Aerodynamic loads acting on a section of a wind turbine blade, twisted at an angle $\beta$ 
The thrust $(T)$ and torque $(Q)$ produced by the normal and tangential forces, respectively, determine the performance of a wind turbine. In order to compare various designs, the thrust and torque of wind turbines can be non-dimensionalized. The following equations define the coefficients of thrust $\left(C_{T}\right)$ and torque $\left(C_{Q}\right)$, respectively.

$$
\begin{gathered}
C_{T}=\frac{T}{1 / 2 \rho A V_{o}^{2}} \\
C_{Q}=\frac{Q}{1 / 2 \rho R A V_{o}^{2}}
\end{gathered}
$$

where $V_{o}$ is the oncoming wind speed, $\rho$ is the fluid density, $A$ is the swept area of the rotor (i.e., $A=\pi R^{2}$ ) and $R$ is the radius of the rotor.

Similarly, the coefficient of power $\left(C_{P}\right)$, another non-dimensional performance parameter, is defined as the ratio between the actual output power obtained from a wind turbine and the theoretical maximum available power $\left(P_{\max }\right)$. Equation 1.3 defines the maximum available power that can be obtained if, in theory, the wind velocity could be reduced to zero.

$$
P_{\text {max }}=1 / 2 \rho A V_{o}^{3}
$$

This maximum coefficient of power is about 16/27 (59.3\%) according to the Betz law. In practice, however, conventional SRWTs can generate up to $75 \%$ to $80 \%$ of the Betz limit due to various losses such as viscous loss, three dimensional loss, and transmission loss [9-12]. 


\subsection{Dual-Rotor Wind Turbines}

Dual-rotor wind turbines (DRWTs) are equipped with two co-axial rotors in a backto-back configuration or with an upwind and a downwind rotor separated by the nacelle, as illustrated in Figure 1.4. Since the shape of the inboard sections of SRWT blades extract little energy from the wind, it is hypothesized that by adding a smaller rotor upstream of a SRWT, more energy can be harvested.

Moreover, since the wake behind a rotor rotates in opposite direction to the rotor itself, more energy can be extracted by having the downwind rotor taking advantage of the wake rotation of the upwind rotor. In other words, counter-rotating DRWT can harvest more energy as compared to co-rotating DRWT [13,14]. The reason why counter-rotating configuration can extract more energy than co-rotating configuration
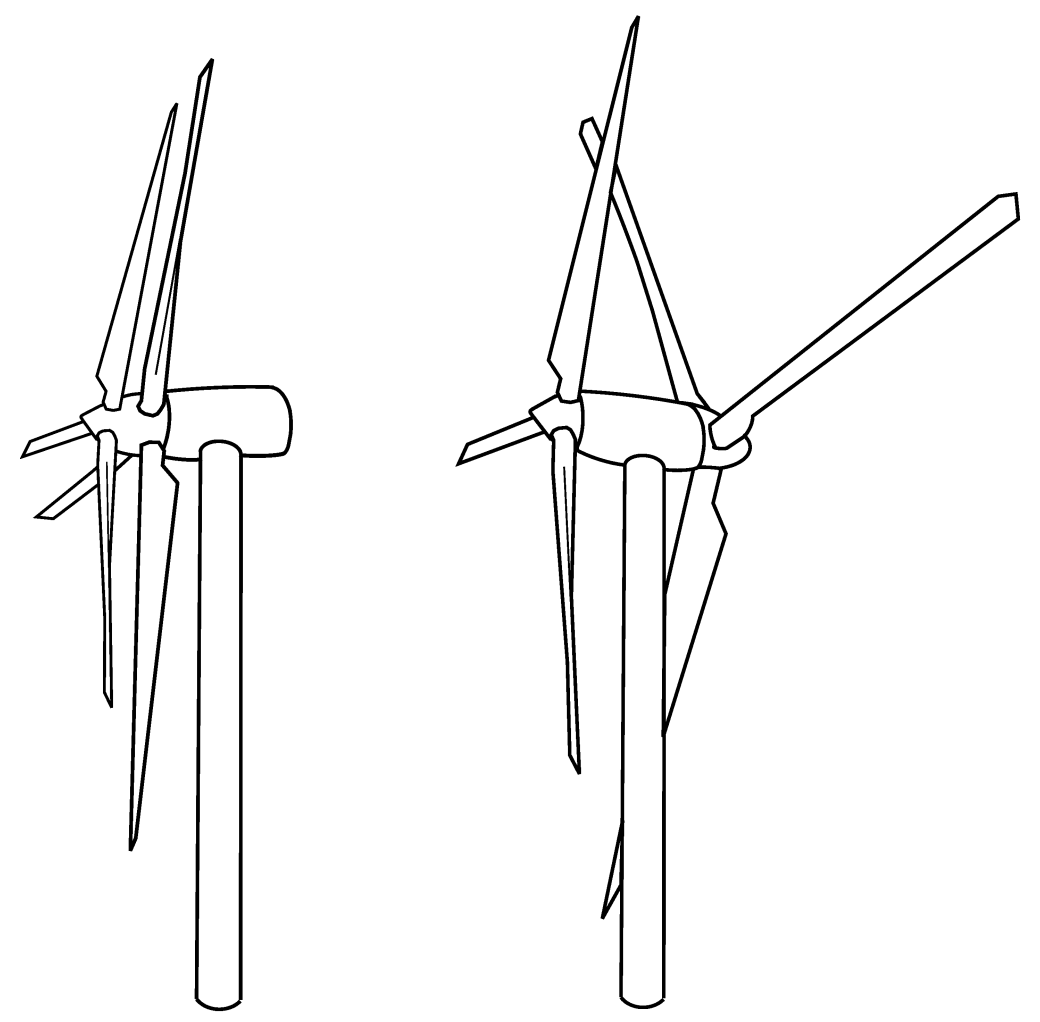

Figure 1.4: Schematic of the two main type of dual-rotor wind turbines 
can be explained by comparing the velocity triangles of the downwind rotor. Figure 1.5 illustrates the velocity triangles for both configurations where $V_{u p}$ represents the effect of the upwind wake as the rotor rotates either in a clockwise or counter-clockwise direction. As can be observed, the swirl velocity $\left(V_{u p}\right)$ from a co-rotating upwind rotor induces a smaller angle of attack $\left(\alpha_{c o}\right)$ thereby decreasing the lift force component on the blade while increasing the relative velocity $\left(V_{c o}\right)$ acting on the blade. On the other hand, in counter-rotating configuration, a larger angle of attack, $\alpha_{\text {counter }}$ (i.e., larger lift coefficient) and lower relative velocity $\left(V_{\text {counter }}\right)$ act on the downwind blade due to the induced swirl from the upwind wake. In short, higher lift coefficient due to a larger angle of attack $\left(\alpha_{\text {counter }}>\alpha_{c o}\right)$ results in a greater torque from the counter-rotating configuration [14].

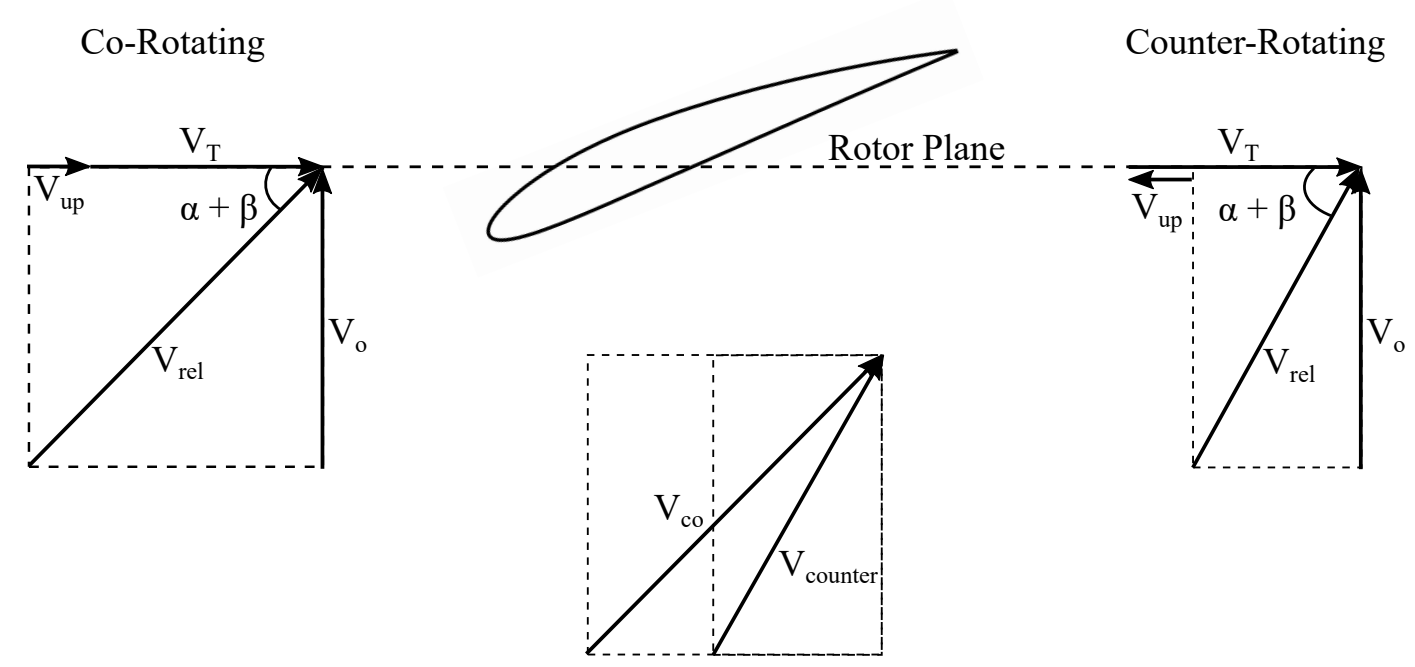

Overlapped Velocity Triangle

$$
\begin{gathered}
\alpha_{\text {co }}<\alpha_{\text {counter }} \\
V_{\text {co }}>V_{\text {counter }}
\end{gathered}
$$

Figure 1.5: Schematic of the velocity triangles for co- (left) and counter-rotating (right) wind turbines 
During the study performed by Jung et al. [15] using a $30 \mathrm{~kW}$ counter-rotating DRWT consisting of an $11 \mathrm{~m}$ diameter main rotor with a $5.5 \mathrm{~m}$ auxiliary upwind rotor, an increase in power output of about $21 \%$ was achieved over a conventional $11 \mathrm{~m}$ diameter rotor turbine at a rated wind speed of $10.6 \mathrm{~m} / \mathrm{s}$. Shen et al. [16] investigated the performance of a counter-rotating DRWT equipped with two Nordtank $500 \mathrm{~kW}$ turbines using the Navier-Stokes solver EllipSys3D. The analysis showed that the turbine can produce $43.5 \%$ more annual energy than a similar SRWT. Habash et al. [5] carried out a wind tunnel study with a model DRWT system equipped with a pair of three-bladed, $23 \mathrm{~cm}$ diameter counter-rotating rotors and a distance between the rotors that varied from $7-54 \mathrm{~cm}$, which resulted in up to $60 \%$ more energy than a SRWT system.

More recently, ISU researchers started investigating DRWT concepts both numerically and experimentally in an attempt to reduce losses near the root of the main rotor (downwind rotor). Utilizing a secondary, smaller, co-axial rotor upstream of the main rotor, Rosenberg et al. [17] studied the effect of varying the secondary rotor diameter, the spacing between the rotors and the secondary rotor tip-speed. The DRWT model used consisted of the NREL $5 \mathrm{MW}$ turbine as the main rotor and an optimized secondary rotor using an inversed BEM method. The analysis was performed using the Reynolds Averaged Navier-Stokes (RANS) solver OpenFOAM coupled with an actuator disk method to obtain an optimized DRWT model. The RANS results showed a net increase of $7 \%$ in power coefficient can be obtained when the swept area of the secondary rotor is $25 \%$ that of the main rotor with a distance separating the rotors of $20 \%$ of the main rotor radius. Furthermore, Ozbay et al. [6] conducted a wind tunnel investigation of DRWTs in either co-rotating or counter-rotating configuration using a model DRWT equipped with two $24 \mathrm{~cm}$ diameter rotors. It was found that the counter-rotating model which harvested approximately $7.8 \%$ more energy than the 
co-rotating model, and produced up to $60 \%$ more wind energy as compared with the SRWT model.

\subsection{Motivation}

The work performed in this thesis is a continuation of the collaboration with ZEC Wind Power Corp. [18], a company partner in Ottawa, who envisioned Dual-Rotor Wind Turbines (DRWTs) as a promising alternative to solar panels and diesel-fueled generators which are generally used in remote locations and areas without access to the electricity grid. As previously mentioned, to capture more energy from the wind, the swept area of conventional HAWTs is growing in size. However, larger turbines come with a number of drawbacks such as increasing weight (therefore, larger stresses on the blades), transportation difficulties, increasing tower height, as well as bigger footprint (more land or sea). A potential alternative to increasing the size of conventional HAWTs, is multi-rotor technology which had fallen out of consideration over the years due to its complexity [19]. In the early 2016, Vestas installed a multirotor prototype consisting of four refurbished V29-225 kW turbines each having a 29m-diameter rotor, attached to a single support structure, near Roskilde in Denmark. The support structure is composed of one central steel tower and two rotor arm assemblies arranged vertically at two levels which can independently rotate around the tower [20].

This thesis aims at providing a preliminary work to establish an optimal configuration for DRWT, in an attempt to generate more power per tower while keeping the blade span within a adequate range. With the low fidelity BEM theory not ca-

pable of simulating DRWT and the more sophisticated Navier-Stokes solver having 
high computational cost, a vortex code that has the ability to compute the performance of dual-rotor wind turbine will be implemented. In addition, to compare the performance of various DRWT models, dimensionless parameters will be derived and tested.

\subsection{Structure of Thesis}

This thesis consists of six chapters. Chapter 2 provides a limited literature survey of the various performance prediction methods such as wind tunnel experiment, blade element momentum theory, vortex theory, and Navier-Stokes solvers. Chapter 3 describes DR_HAWT, the vortex filament code implemented in this thesis, including the mathematical formulations as well as the structure of the code. Chapter 4 details various test cases performed to verify if the code was well implemented and the validation of DR_HAWT against two well-known experiments, namely the NREL Phase VI and MEXICO wind turbines. Chapter 5 describes the dimensionless parameters derived in this work and the parametric study performed to investigate their validity as well as any potential effect of varying each parameter. Finally, Chapter 6 outlines the results drawn from the parametric study and provides some suggestions for future work. 


\section{Chapter 2}

\section{Review of Performance Prediction Methods}

During the design process, the aerodynamic performance of wind turbines are typically analyzed using numerical models ranging from models based on the Blade Element Momentum (BEM) theory to more sophisticated unsteady aerodynamic models using either a Prescribed-Wake Method (PWM) or a Free-Wake Method (FWM) to Computational Fluid Dynamics (CFD) models [21-27]. Despite being widely used nowadays, these models were built on empirical assumptions and validated based on specific wind turbines. Due to this drawback, numerical modeling does not provide enough flexibility to accurately design better performing wind turbines, however, with the increasing research and development in numerical models, vortex wake theories and CFD models based on Euler and Navier-Stokes solutions are promising prediction methods for the future of wind turbine design [12].

The BEM approach is generally employed by wind turbine manufacturers due to its fast computational runtime which makes it well suited for assessing new design performance. A summary of relevant performance prediction softwares for wind turbines available in the literature is compiled in Table 2.1. It should be noted that for 
Table 2.1: Summary of performance prediction software for wind turbines

\begin{tabular}{lllll}
\hline Author & Code Name & Affiliation & Method $^{1}$ & Reference \\
\hline Marten & QBlade & TU Berlin & BEM, FWM & {$[28,29]$} \\
Jonkman & AeroDyn & NREL & BEM & {$[30]$} \\
Hassan & Bladed & DNV_GL & BEM & {$[31]$} \\
van Garrel & AWSM & ECN & FWM & {$[32,33]$} \\
Sant & HAWT-FWC & TUDelft & FWM & {$[34]$} \\
Kloosterman & - & TUDelft & FWM & {$[35]$} \\
Sebastian & WInDs & UMass & FWM & {$[36]$} \\
Abedi & - & Chalmers & PWM, FWM & {$[37]$} \\
Rosenberg & - & Iowa & PWM & {$[38]$} \\
Voutsinas & GENUVP & NTUA & FWM & {$[39]$} \\
Coton & HAWTDAWG & UGlasgow & PWM & {$[40]$} \\
Strickland & VDART3 & Sandia Lab & FWM & {$[41]$} \\
Michelsen & EllipSys3D & DTU/Ris $\varnothing$ & RANS & {$[42-44]$} \\
\hline
\end{tabular}

1. Blade Element Momentum theory (BEM), Free-Wake Method (FWM), Prescribed-Wake Method (PWM), Reynolds Averaged Navier-Stokes (RANS)

each code, only the name of the first author is shown in Table 2.1 for compactness. A review of other commercial softwares is presented by Molenaar in Ref. [45]. The following sections describe various types of codes (BEM, vortex models, CFD models) as well as the commonly used validation experiments.

\subsection{Wind Tunnel Experiments}

A significant number of experiments which were performed to characterize the performance of wind turbines, are available in the literature (see Refs. $[23,46]$ ). Two well-known wind turbine experiments which were carried out in a controlled environment, are commonly used to validate performance codes - the National Renewable 
Energy Laboratory (NREL) Unsteady Aerodynamics Experiment (UAE) [47-49] and the Model Experiments in Controlled Conditions (MEXICO) project [50,51]. Figure 2.1 shows the NREL Phase VI and MEXICO wind turbines mounted in their respective wind tunnel.

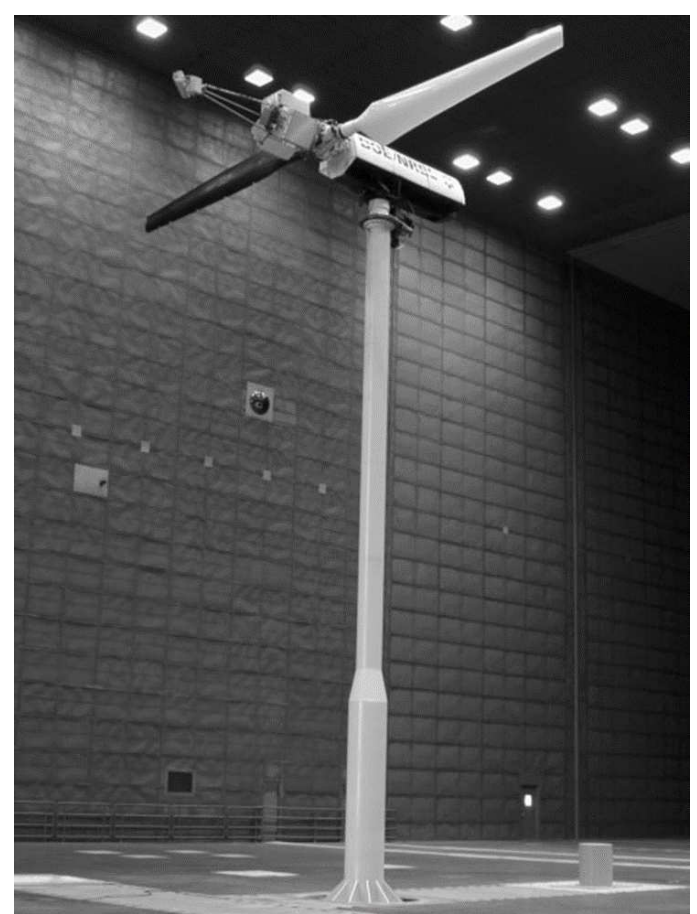

(a) NREL Phase VI

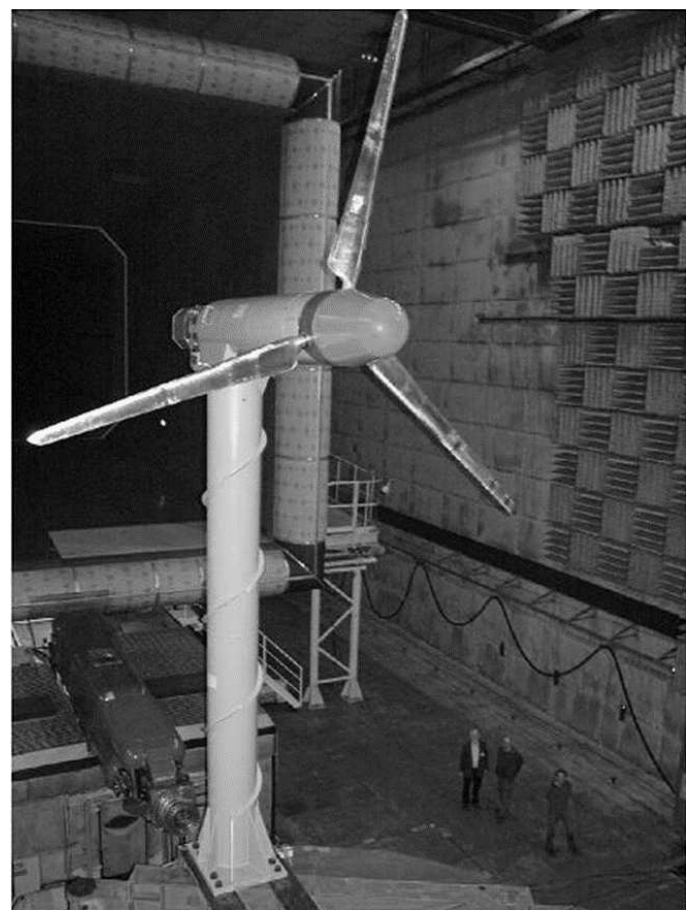

(b) MEXICO

Figure 2.1: NREL Phase VI turbine mounted in NASA Ames $24.4 \mathrm{~m} \times 36.6 \mathrm{~m}$ wind tunnel (left) [49] and MEXICO turbine mounted in DNW $9.5 \mathrm{~m} \times 9.5 \mathrm{~m}$ wind tunnel (right) [51]

\subsubsection{NREL Phase VI Wind Turbine}

The Unsteady Aerodynamics Experiment (UAE) was carried out throughout 1999 and 2000 in the NASA Ames $24.4 \mathrm{~m} \times 36.6 \mathrm{~m}$ wind tunnel using the $10 \mathrm{~m}$ diameter NREL Phase VI wind turbine. This twisted, two-bladed, stall-controlled rotor consisting of mainly S809 airfoil sections as shown in Figure 2.2, was tested under 14 different turbine configurations. More details about the geometry of this turbine 
will be provided in Section 4.3. The objective of this experiment was to provide accurate and reliable quantitative information on full-scale, 3D aerodynamic behaviour of HAWTs. These measurement data have contributed to the development and validation of engineering models for the design of better performing wind turbines. The reader is referred to Refs. $[47,48]$ for a complete description of the test plan, model geometry, instrumentation, and calibration procedures as well as a summary of the experimental results.

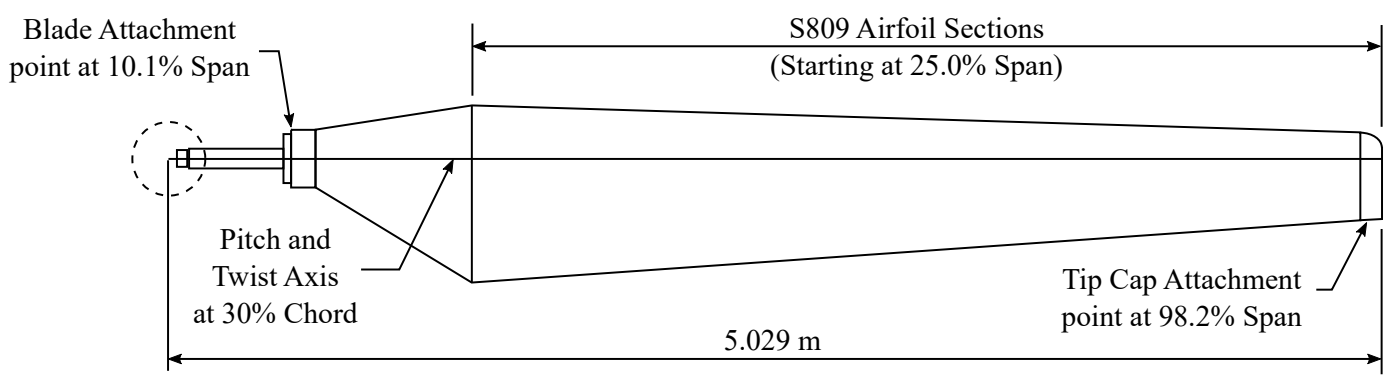

Figure 2.2: NREL Phase VI blade geometry. Adapted from Ref. [47]

Following the experiment, a blind comparison was performed with 30 academic and industrial wind turbine experts using prediction codes ranging from blade element models, prescribed-wake models, free-wake models, and full Navier-Stokes codes. A summary of the participants who took part of the blind comparison and a description of their code can be found in Ref. [48]. Figure 2.3 shows the Sequence S measurements and some of the best performance predictions resulting from the blind comparison. As can be seen, the results demonstrated large deviations in the prediction accuracy. In $0^{\circ}$ yaw, steady-state, and pre-stall conditions, the power predictions ranged from $25 \%$ to $175 \%$ of the measured data. On the other hand, in the stall regimes, where dynamic stall and unsteady blade loading are present, the power prediction ranged from $30 \%$ to $275 \%$ of the measured data [48]. 


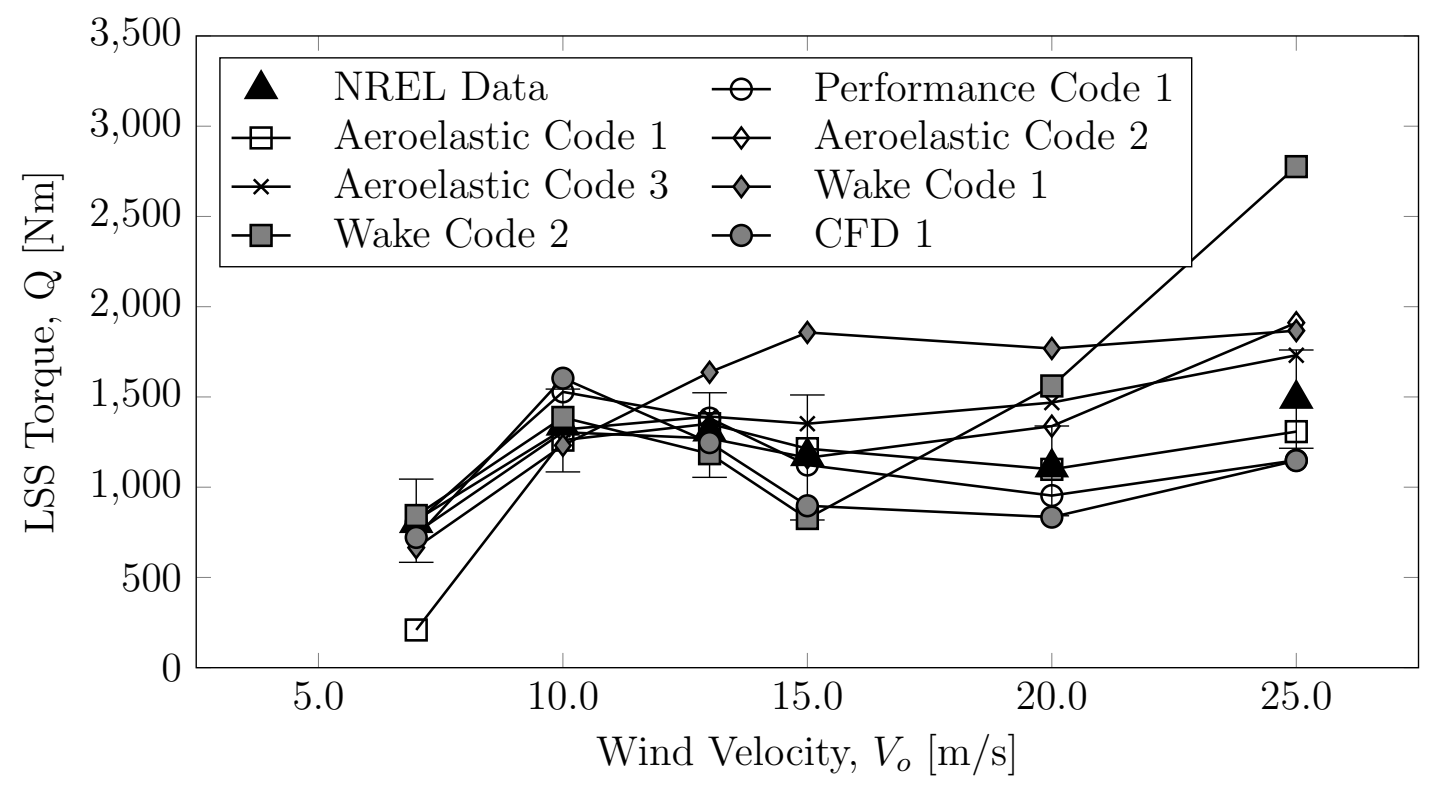

Figure 2.3: NREL Phase VI blind comparison results extracted from Ref. [48]. Experimental data provided by Dr. Schreck [52]

\subsubsection{MEXICO Project}

Similar to the UAE, the MEXICO Project was performed to build an experimental database as a means of validating engineering models. The experiment consisting of blade pressure distributions, loads and flow field measurements, was conducted in 2006. The $4.5 \mathrm{~m}$ diameter model rotor and operating conditions were designed to complement the UAE database. This three-bladed rotor employing three different aerodynamic profiles (DU91-W2-250, RISOE-A1-21 and NACA 64-418), as shown in Figure 2.4, was placed in the $9.5 \mathrm{~m} \times 9.5 \mathrm{~m}$ LLF (large scale low speed facility) of the German Dutch Wind Tunnel (DNW) in the Netherlands. To trigger the laminar to turbulent flow transition, a $0.25 \mathrm{~mm}$ thick zig-zag tape placed at $5 \%$ of the chord was applied on both pressure and suction sides of the turbine blades. The measurements were carried out under several flow conditions, including 2 different rotational speeds (324 rpm and $424 \mathrm{rpm}$ ), wind speeds ranging from $10 \mathrm{~m} / \mathrm{s}$ to $30 \mathrm{~m} / \mathrm{s}$, pitch angles ranging from $-5.3^{\circ}$ to $1.7^{\circ}$, and yaw-inflow angles ranging from $0^{\circ}$ to $45^{\circ}$. The reader 


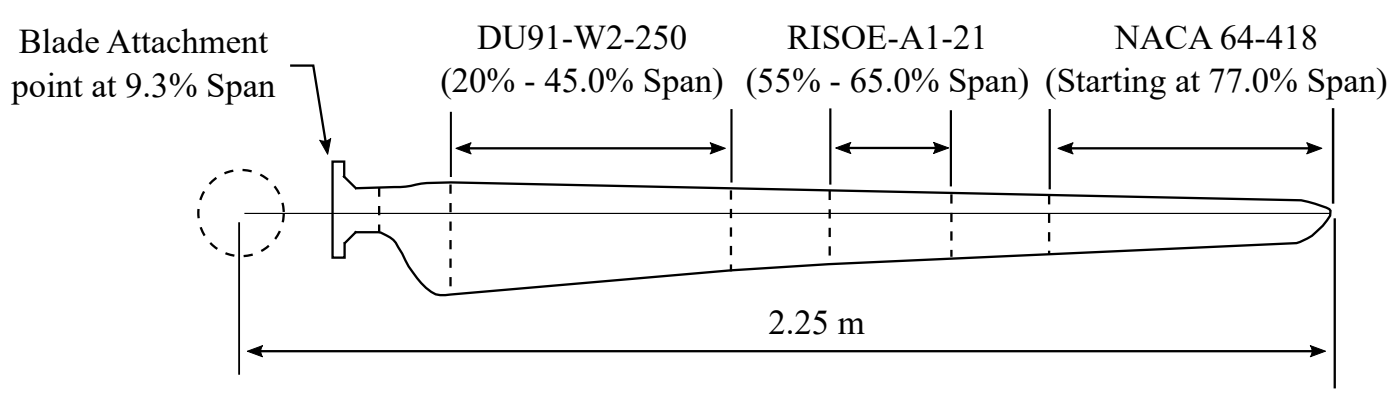

Figure 2.4: MEXICO blade geometry

is referred to Refs. $[50,51,53,54]$ for more information regarding the setup, rotor configuration, and measurements performed.

Both yawed and non-yawed measurement cases were compared with different types of codes including BEM, vortex model, and CFD. To reduce errors, the blade geometry was provided as IGES and STEP files to CFD participants. Moreover, 2D airfoil wind tunnel experiments were performed at Delft University of Technology and Ris $\varnothing$ National Laboratory thereby creating a polar database to be used as an input to codes using engineering approximations (e.g., BEM). The same type of zig-zag tapes were applied for the 2D airfoil experiments although the thickness of the strips might vary between experiments. Figures 2.5 and 2.6 show the torque and thrust results, respectively, from various prediction codes compared to the experimental data of the non-yawed case. A description of each code can be found in Ref. [51].

\subsection{Blade Element Momentum (BEM) Theory}

The Blade Element Momentum (BEM) theory, developed by Glauert in 1935 [56], is the simplest and most commonly used prediction tool for wind turbines. This method uses a combination of two mathematical methods - blade element theory 


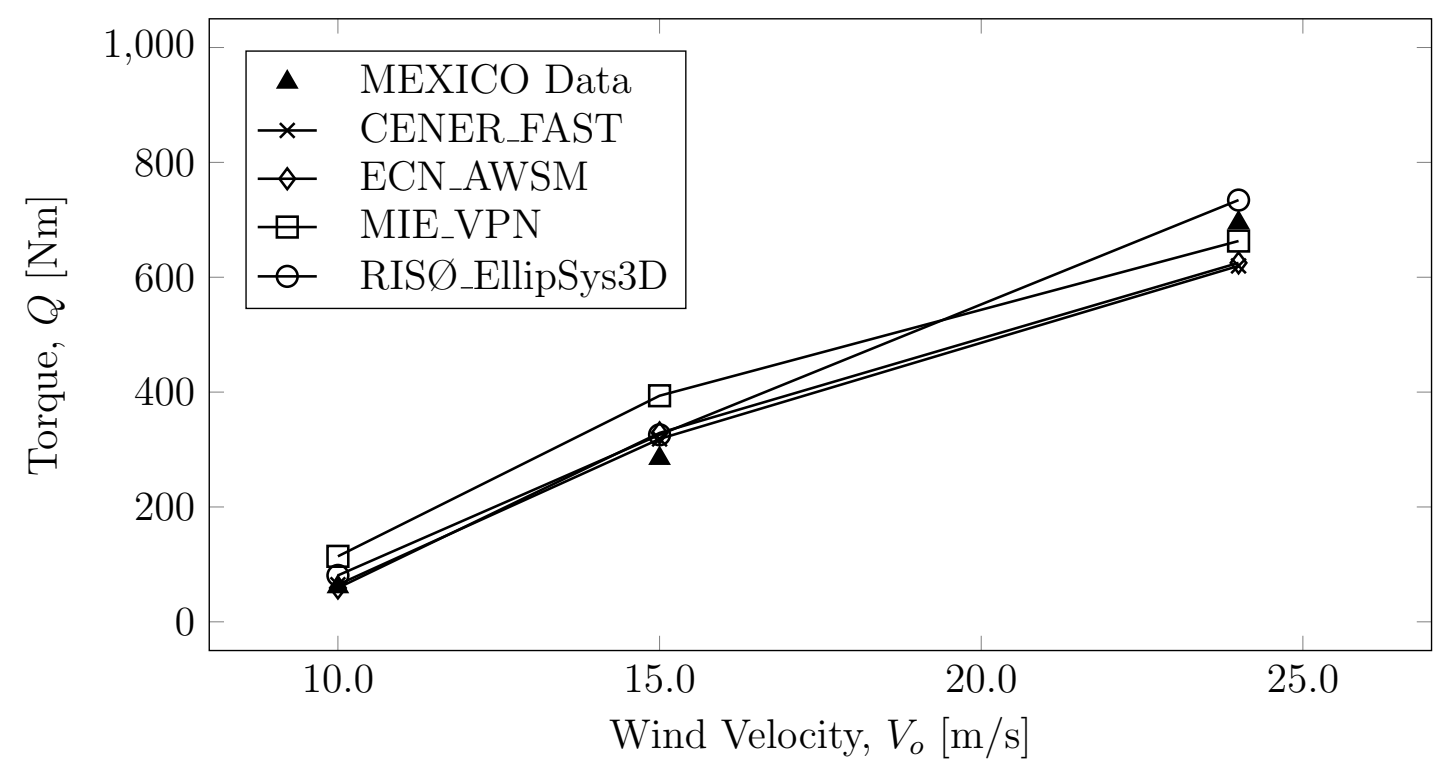

Figure 2.5: MEXICO torque measurements compared with the prediction from various codes (extracted from Ref. [51]). Experimental data provided by Dr. Schepers $[55]$

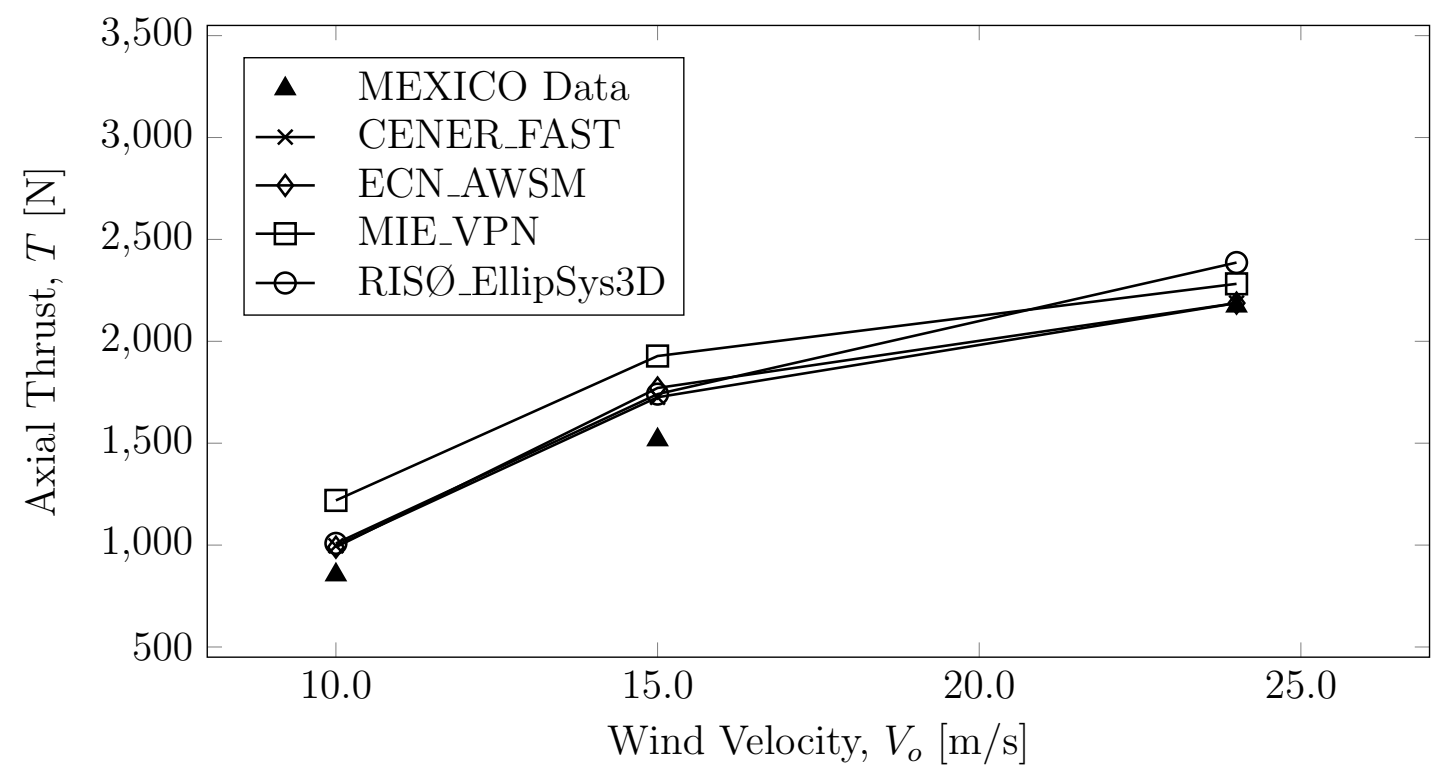

Figure 2.6: MEXICO axial thrust measurements compared with the prediction from various codes (extracted from Ref. [51]). Experimental data provided by Dr. Schepers [55] 
and momentum theory - along with various empirical approximations to evaluate the loads acting on discretized radial elements of a wind turbine blade. Typical corrections that are applied to BEM methods are: i) blade tip and root effects, ii) turbulent wake state, iii) dynamic inflow, iv) dynamic stall, and v) 3D corrections [45].

The momentum theory is a one-dimensional (1D) model in which the flow is assumed to be inviscid, incompressible, and axisymmetric. It basically computes the conservation of mass, momentum, and energy over control volumes fixed by stream tubes to solve for the loads acting on the blade elements. In other words, the induced velocities from the momentum lost in the flow can be calculated using the momentum theory and can be modeled using both the axial and radial inflow [9-11] or solely based on the axial inflow [57]. These induced velocities which are usually specified as induction factors, affect the inflow in the rotor plane and therefore also affect the loads acting on the blades. On the other hand, the blade element theory assumes that blades can be divided into small independent elements thereby operating as $2 \mathrm{D}$ airfoils. This allows the lift and drag forces on each blade elements to be evaluated based on the local 2D airfoil characteristics, angle of attack and relative velocity. A detailed derivation of the BEM theory can be found in Refs. [9-11].

The BEM method provides a simple and fast way to compute the performance of wind turbines, however, in situations such as yawed flow and unsteady aerodynamics (particularly dynamic inflow effects), this method lacks accuracy [24,58]. Although there are corrections and models that can be integrated into the BEM algorithm to account for these effects, the use of induced velocities compared to induction factors provide a more accurate performance prediction. 


\section{$2.3 \quad$ Vortex Methods}

As previously mentioned, although computationally more expensive compared to BEM methods, vortex models provide more accurate performance predictions due to better spatial and temporal representation of the wake. In this method, the wind turbine blades and wake (spanwise and trailing vortices) are modeled by either vortex particles (also known as vortex blobs) [39], vortex lines (straight or curved filaments) $[32,36,41]$, vortex panels [37], or a hybrid method $[29,59]$. Based on the Kutta-Joukowski Theorem, the vortex strength of an element on the blade (commonly known as bound circulation) is determined from the lift generated by the local inflow field $[23,24]$. This flow field is related to the oncoming wind, blade, and induced velocities which are determined using the Biot-Savart law (see Section 3.6). As the blades passes through the flow field, vorticity is generated in the wake due to spatial and temporal variations in bound circulation. In other words, the resulting wake consists of spanwise vortices generated by spatial variations of the bound circulation along the blade and trailing vortices generated by the temporal variations as the blades rotate. This method is considered to be an efficient and flexible way to represent complex wake structures $[35,57,60]$.

The convection of the wake can either be prescribed or free. The former method involves specifying the wake geometry based on analytical or empirical solutions. On the other hand, a free-wake geometry is obtained from first principle thereby allowing the vortices in the wake to interact with themselves (self-induced effect), with other vortices (mutually induced effect) or with the blades [57]. The free-wake method, which is the most computationally expensive vortex method, is able to predict the wake geometry and loads more accurately than the prescribed-wake because of less restrictive assumptions. After obtaining the positions and strengths of the vortices, 
the loads acting on the blades and therefore the performance of the wind turbine can be evaluated. A review of vortex methods and their applications is presented by Stock in Ref. [61].

\subsection{CFD Models}

Computational Fluid Dynamics (CFD) models, which rely on the first principles of conservation of mass, momentum, and energy, are based on numerical solutions to the Euler and Navier-Stokes equations. CFD is, in theory, the most comprehensive way to analyze the entire flow field around any body in steady or unsteady flow such as wind turbines. In practice, however, these codes are computationally very expensive and require large amount of memory. In addition, CFD models have various numerical issues such as turbulence modeling and wake diffusion [22].

There are many commercially available CFD softwares that have demonstrated a clear ability to predict the forces acting on wind turbine blades. The Reynolds Averaged Navier-Stokes equations (RANS) are commonly solved for wind turbine applications with various turbulence models such as the k-omega SST (Shear Stress Transport) model of Menter [62], the Spalart-Allmaras model [63], and the BaldwinBarth model [64]. All RANS models, however, cannot model large separations accurately. An alternative to RANS simulations is Large Eddy Simulation (LES) which provides better physical representation of the eddy dynamics in separate flows, yet is limited to moderate Reynolds numbers. Detached Eddy Simulation (DES), on the other hand, is a hybrid technique which provides a more accurate and affordable option to RANS and LES. DES combines the capability of RANS to model boundary layers using "attached" eddies, and LES to model separated regions using "detached" eddies [65]. 


\subsection{Comparison of Prediction Tools}

As previously discussed, the blade element momentum theory (BEM) provides a simple and fast method to predict the performance of a single-rotor wind turbine (SRWT). However, this method cannot accurately capture all the flow phenomena which occurs in the wake of a HAWT and therefore, cannot be used to simulate a dual-rotor wind turbine. CFD, which has the ability to accurately simulate the entire flow field around a dual-rotor wind turbine, is too computationally expensive and therefore, impractical for parametric studies. Free-wake method (FWM), on the other hand, has superior capability to predict aerodynamic performance of wind turbine compared with the BEM method $[32,66]$. In addition, this method, which requires much less computational time than CFD simulations, can be used for parametric studies.

To highlight the accuracy and computational cost of each method, the QBlade, DR_HAWT and EllipSys3D codes (BEM, FWM, and CFD, respectively) were used to compute the performance of the NREL Phase VI. Figure 2.7 compares the torque measurements to the computed results from the three codes. The data for QBlade and Ellipsys3D were extracted from Ref. [29] and Ref. [67], respectively, whereas the results computed in DR_HAWT will be described in Section 4.3. As can be seen, all three methods show good agreement with a maximum of $30 \%$ difference as compared to the measured data in the pre-stall and stall regimes $(7 \mathrm{~m} / \mathrm{s}$ to $15 \mathrm{~m} / \mathrm{s})$.

Marten et al. computed the performance of the two-bladed Phase VI rotor using 18 discretized elements. Although the computational time was not provided in Ref. [29], the computational cost of BEM simulations are known to be in the order of seconds. As part of the UAE blind comparison, Sørensen et al. used EllipSys3D which is a RANS solver, to perform steady and unsteady computations. In these simulations, 


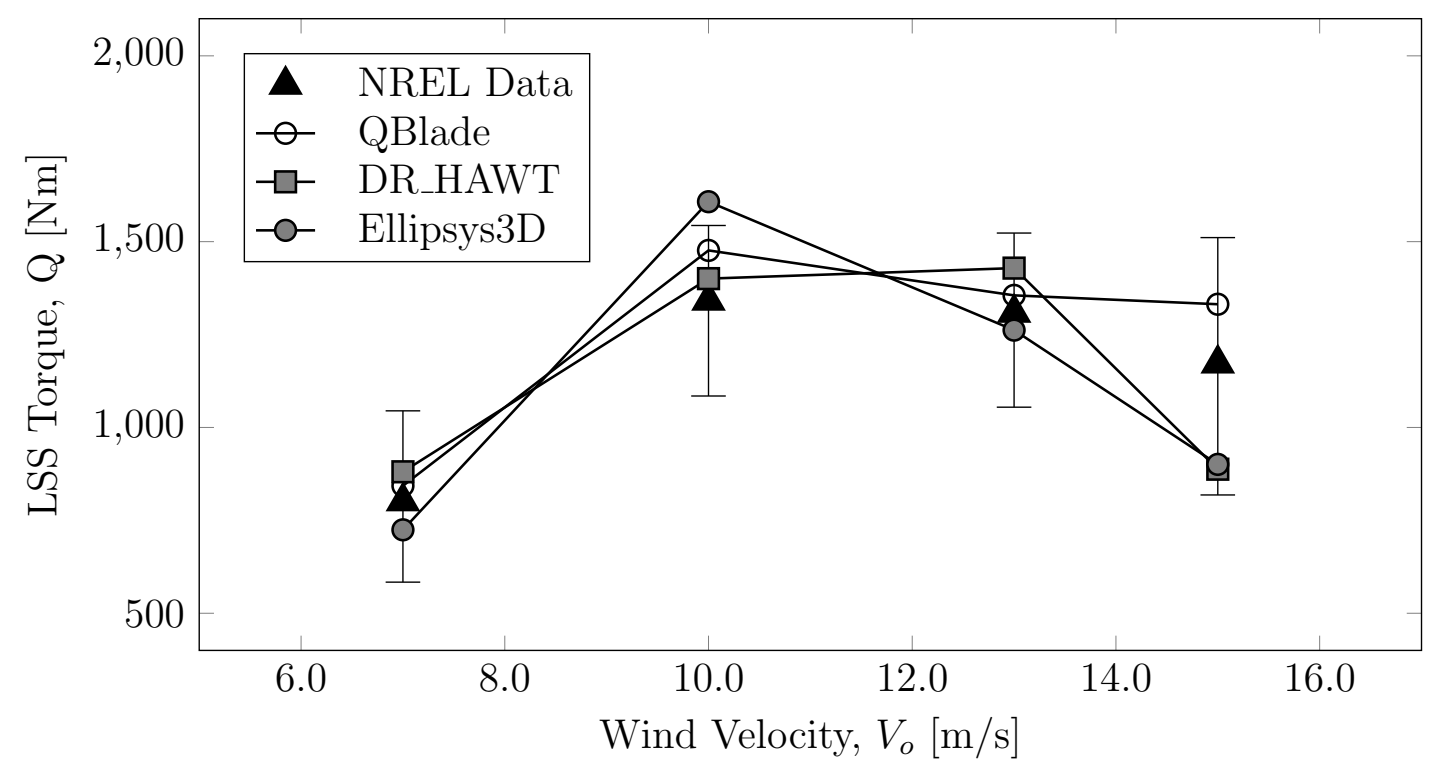

Figure 2.7: Power prediction of the NREL Phase VI wind turbine using the QBlade, DR_HAWT, and Ellipsys3D codes

the tower and nacelle were neglected, and a single rotor blade was modeled. With over 3 million cells used to model the rotor and computational domain, the steady state simulation required about 30 hours on average using 4 CPUs [67]. DR_HAWT, on the other hand, required 15-30 mins to compute the two-bladed Phase VI rotor discretized with 26 elements using 8 CPUs (more details are provided in Section 4.3).

To summarize, a free-wake method was determined to be better suited for a dualrotor wind turbine parametric study. It provides a relatively fast computational cost and possesses the ability to accurately model rotor wakes as compared to BEM and CFD methods. 


\section{Chapter 3}

\section{DR_HAWT: Vortex Filament Code}

This chapter describes the aerodynamic model used in DR_HAWT (Dual-Rotor Horizontal-Axis Wind Turbine code) for the aerodynamic performance prediction of a single or dual-rotor horizontal axis wind turbine. The general procedure used is based on the code devised by Strickland et al. for the Darrieus Vertical Axis Wind Turbine $[41,68]$. In this three-dimensional (3D) free vortex model, the rotor blades are divided into a number of elements along their span and each element is replaced by a bound vortex filament based on the Lifting Line Theory (LLT). The vortex filaments are then shed at each time step in terms of spanwise and trailing vortices. These shed vortices are free to convect in the fluid domain thereby providing a suitable spatial and temporal representation of the wake. The aerodynamic loads acting on the blades can then be determined following the Blade Element Theory (BET).

The material presented in the following sections will allow the reader to understand the mathematical formulations implemented in DR_HAWT including any correction factors employed. 


\subsection{Governing Equations}

An incompressible, inviscid flow with no body forces, is governed by the conservation of mass and momentum, given by:

$$
\begin{gathered}
\nabla \cdot \mathbf{v}=0 \\
\frac{D \mathbf{v}}{D t}=\frac{\delta \mathbf{v}}{\delta t}+\mathbf{v} \cdot \nabla \mathbf{v}=-\frac{\nabla p}{\rho}
\end{gathered}
$$

where $\mathbf{v}, t, p$, and $\rho$ denote the velocity vector, time, pressure, and fluid density, respectively and $\nabla$ is the gradient operator. The inviscid vorticity transport can be obtained by taking the curl of Equation 3.2, giving

$$
\frac{D \zeta}{D t}=(\zeta \cdot \nabla) \mathbf{v}
$$

where the vorticity $\zeta$ is a measure of the local rotation or spin of a fluid element about an axis through the element and is defined as the curl of the velocity $(\zeta=\nabla \times \mathbf{v})$. The term on the right-hand side of Equation 3.3 denotes the tilting and stretching of vorticity. This equation is the basis of the Helmholtz's theorem, where the strength of a vortex tube remains constant along its length and does not vary with time. Vortex lines, which are defined as the line fields that are parallel to the local vorticity vector, passing through a closed curve in space form a vortex tube. A vortex filament is then defined as a vortex tube with infinitesimal cross-sectional area $[69,70]$.

Circulation (also referred to as vortex strength) is defined as the negative of the line integral of velocity around a closed curve in the flow, given by:

$$
\Gamma=-\oint_{C} \mathbf{v} \cdot \mathbf{d} \mathbf{s}
$$




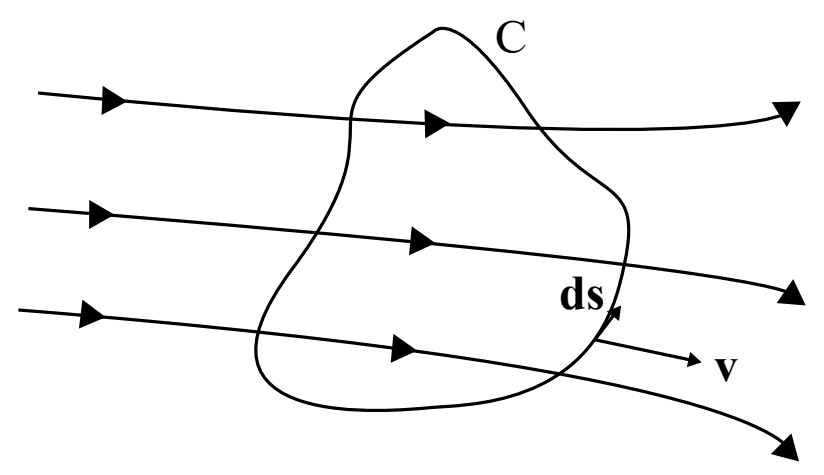

Figure 3.1: Definition of circulation

where $\mathbf{v}$ and $\mathbf{d s}$ are the velocity vector and infinitesimal line segment, respectively, at a point on the closed curve $\mathrm{C}$, as illustrated in Figure 3.1. Therefore, the time rate of change of the circulation of this fluid curve $\mathrm{C}$ gives

$$
\frac{D \Gamma}{D t}=-\oint_{C} \frac{D \mathbf{v}}{D t} \cdot \mathbf{d} \mathbf{s}
$$

When substituting Equation 3.2 into Equation 3.5, the Kelvin's theorem is obtained where the circulation about any closed path moving with the fluid is a constant.

$$
\frac{D \Gamma}{D t}=0
$$

\subsection{Coordinate System}

As previously mentioned, each blade is discretized into elements, defined by unit vectors in the spanwise (s), chordwise (c), and normal (n) directions, located at their aerodynamic center (AC), as shown in Figure 3.2. It should be noted that the chordwise vector lies on the rotor plane and therefore, does not take into account 


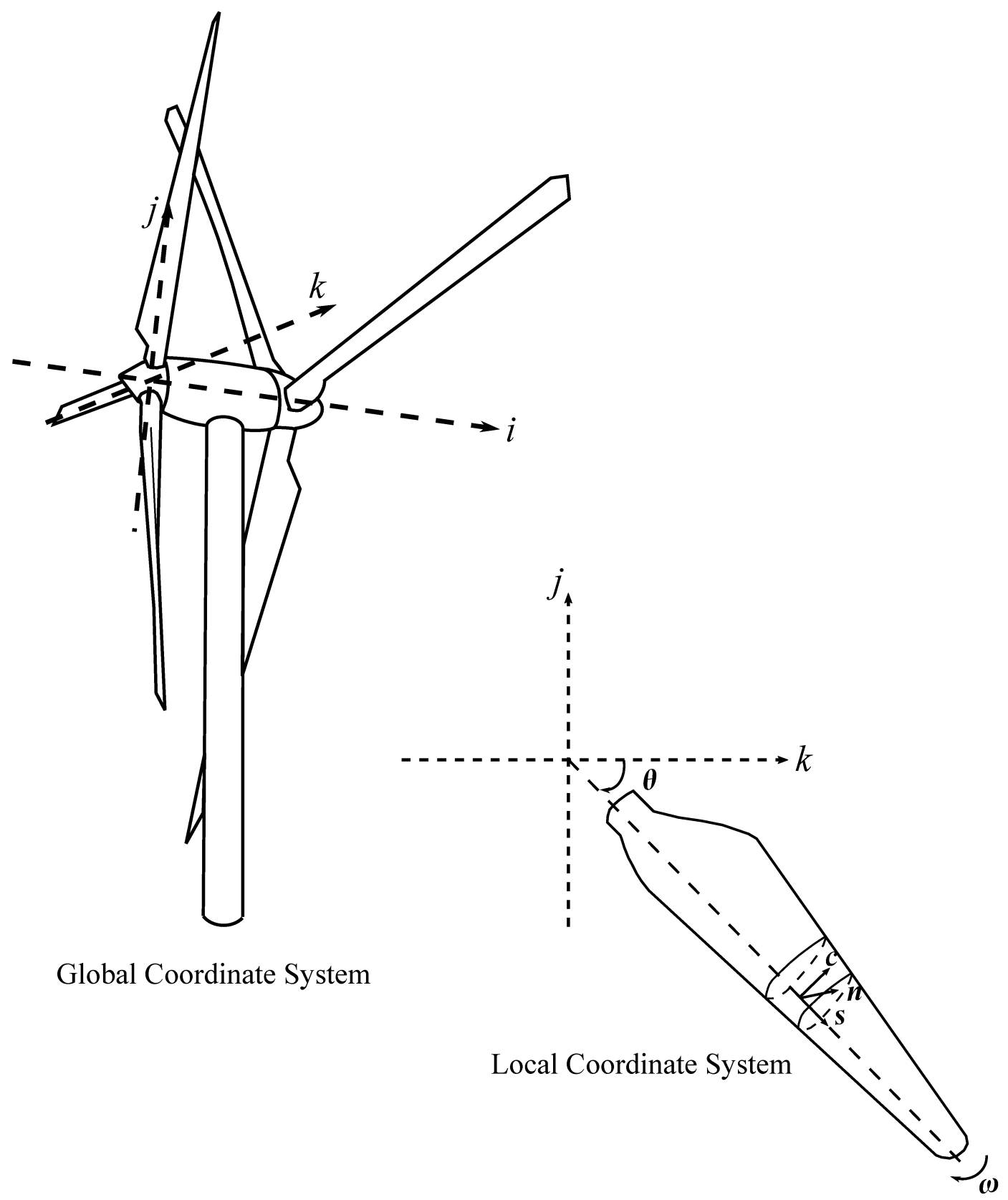

Figure 3.2: $3 \mathrm{D}$ model representing wind turbine in DR_HAWT 
blade twist and pitch. In this thesis, these unit vectors are being identified as local coordinate system (LCS) which allows for the calculation of the local flow conditions (Re, AOA) and the aerodynamic coefficients $\left(\mathrm{C}_{L}, \mathrm{C}_{D}\right)$. Equations $3.7-3.9$ define the LCS in a generalized form.

$$
\begin{gathered}
\mathbf{s}=\frac{\left(x_{i+1}-x_{i}\right) \mathbf{i}+\left(y_{i+1}-y_{i}\right) \mathbf{j}+\left(z_{i+1}-z_{i}\right) \mathbf{k}}{\left[\left(x_{i+1}-x_{i}\right)^{2}+\left(y_{i+1}-y_{i}\right)^{2}+\left(z_{i+1}-z_{i}\right)^{2}\right]^{1 / 2}} \\
\mathbf{c}=\cos \theta_{B} \mathbf{j}+\sin \theta_{B} \mathbf{k} \\
\mathbf{n}=\mathbf{c} \times \mathbf{s}
\end{gathered}
$$

where the subscript $i$ identifies the element number along the blade and $\theta_{B}$ is the blade azimuth angle. $x, y$ and $z$ are the coordinates of the AC of any section of the blades defined in the global coordinate system (GCS). A schematic of the wind turbine model used in DR_HAWT is illustrated in Figure 3.2. As can be seen, the origin of GCS is located at the center of the upwind hub with the $\mathbf{j}$ - and $\mathbf{k}$-axes in the vertical and horizontal directions, respectively. The $\mathbf{i}$-axis is oriented parallel to the axis of rotation with the positive axis in the direction of the wind during zero yaw condition. Note that either clockwise or counter-clockwise rotation can be modeled where a '-1' factor is introduced in Equations 3.8 and 3.9 to account for counterclockwise rotation. Equations 3.10 - 3.13 define the coordinates and azimuth angle of an element in a generalized form.

$$
\theta_{B}=\frac{2 \pi}{N B}(B N-1)+\theta
$$




$$
\begin{gathered}
x=0 \text { or } L \\
y=-r \sin \theta_{B} \\
z=r \cos \theta_{B}
\end{gathered}
$$

where $r$ is the radial distance from the center of the hub to the AC of the blade element, $N B$ is the number of blades for each rotor, $B N$ is the blade number and $\theta$ is the angle rotated by blade 1 from the positive $\mathbf{k}$-axis in the clockwise direction. Since it is assumed that the blades are rigid, the aerodynamic centers of the sections of the blade follow the perimeter of a circle with radius, $r$ in one revolution. In other words, all the ACs of the same rotor lie along the rotor plane. Therefore, for all the blade elements of the same rotor, a fixed value of $x=0$ for the upwind rotor, and $x=L$ where $L$ is the distance between the rotors in the positive $\mathbf{i}$-direction, for the downwind rotor is considered.

\subsection{Element Bound Vorticity}

Based on the global coordinate system described in Section 3.2 and the velocity vectors acting on the blade element (see Figure 3.3), the relative velocity, $\mathbf{V}_{\text {rel }}$ of each blade element can be evaluated by summing the blade rotational velocity, $\mathbf{V}_{T}$, free stream velocity, $\mathbf{V}_{o}$, and the induced velocity caused by the vortex filaments, $\mathbf{V}_{\text {ind }}$. The approach used for calculating the velocity induced by the vortex filaments 


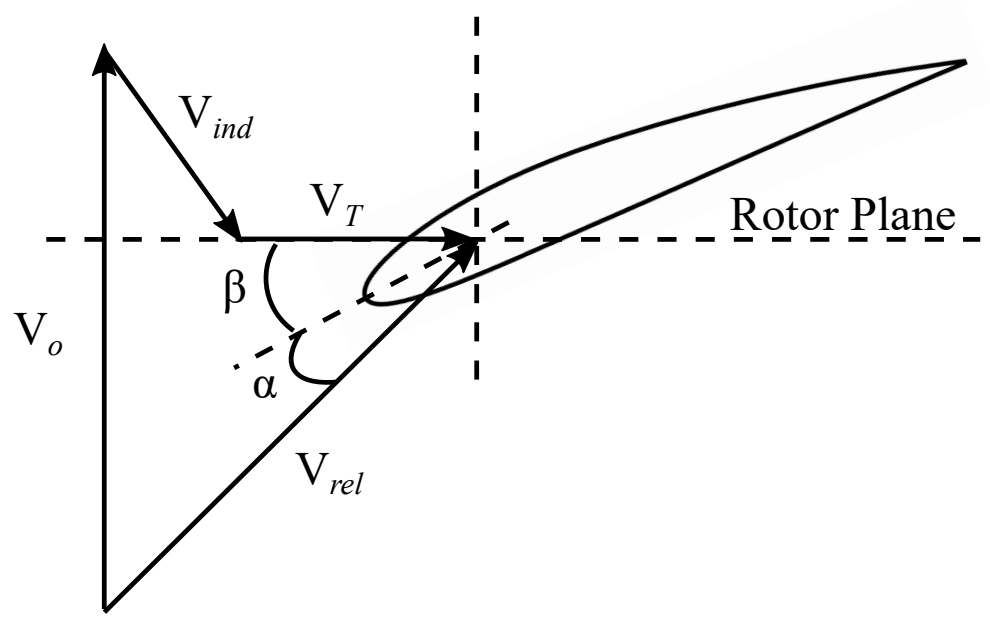

Figure 3.3: Velocity components acting on blade element

is described in Section 3.6. The vector sum of the relative velocity is given below:

$$
\mathbf{V}_{r e l}=\left(\mathbf{V}_{i n d, x}+\mathbf{V}_{o}\right) \mathbf{i}+\left(\mathbf{V}_{i n d, y}+\mathbf{V}_{T} \cos \theta_{B}\right) \mathbf{j}+\left(\mathbf{V}_{i n d, z}+\mathbf{V}_{T} \sin \theta_{B}\right) \mathbf{k}
$$

Equations 3.15 and 3.16 define the Reynolds number (Re) and the angle of attack $(\alpha)$ at each element section using vector manipulation of the relative velocity.

$$
\begin{gathered}
R e=\frac{\left|\mathbf{V}_{r e l}\right| c}{\nu} \\
\alpha=\tan ^{-1}\left(\frac{\mathbf{V}_{r e l} \cdot \mathbf{n}}{\mathbf{V}_{r e l} \cdot \mathbf{c}}\right)-\beta
\end{gathered}
$$

where $c$ is the local chord length, $\beta$ is the sum of the local twist and pitch angle, and $\nu$ is the kinematic viscosity of the fluid. Knowing the local Reynolds number and angle of attack, the lift and drag coefficients are linearly interpolated from lookup tables consisting of experimental or numerical (e.g., XFOIL) aerodynamic polars. 
XFOIL [71] which employs a viscid/inviscid panel method, is used to compute the lift and drag coefficients. Although the latter method over-predicts lift and underpredicts drag, especially under post-stall conditions [72-74], its low computational runtime, allows new designs to be tested quickly.

Based on Prandtl's lifting line theory, each blade element is associated with a bound vortex filament of strength, $\Gamma_{B}$. This strength can be expressed using the Kutta-Joukowski theorem as follows:

$$
\Gamma_{B}=\frac{1}{2} C_{L} c\left|\mathbf{V}_{r e l}\right|
$$

Since the bound vortex strength is a function of the relative velocity, which takes into account the induced velocity, a predictor corrector method is employed for which the bound vortex strength is predicted based on the induced velocities from the previous timestep. The predicted strength is then used to correct the induced velocities which in turn corrects the bound vortex strength. This process is repeated until the differences between the predicted and corrected values are below $1 \times 10^{-3}$. Figure 3.4 illustrates the procedure for the predictor corrector method employed in this code.

\section{$3.4 \quad$ Vortex Shedding}

Figure 3.5 illustrates the representation of blade elements with the wake which is made up of vortex filaments. Each vortex strength, shown in Figure 3.5 is associated with a superscript and a subscript notation where the superscript denotes the blade element end section and the subscript denotes the time step. In other words, for a timestep $N T$, the bound vortex strength of an element $i$ is written as $\Gamma_{N T}^{i}$. As the blade rotates, vortices are shed in the form of spanwise and trailing vortex filaments 


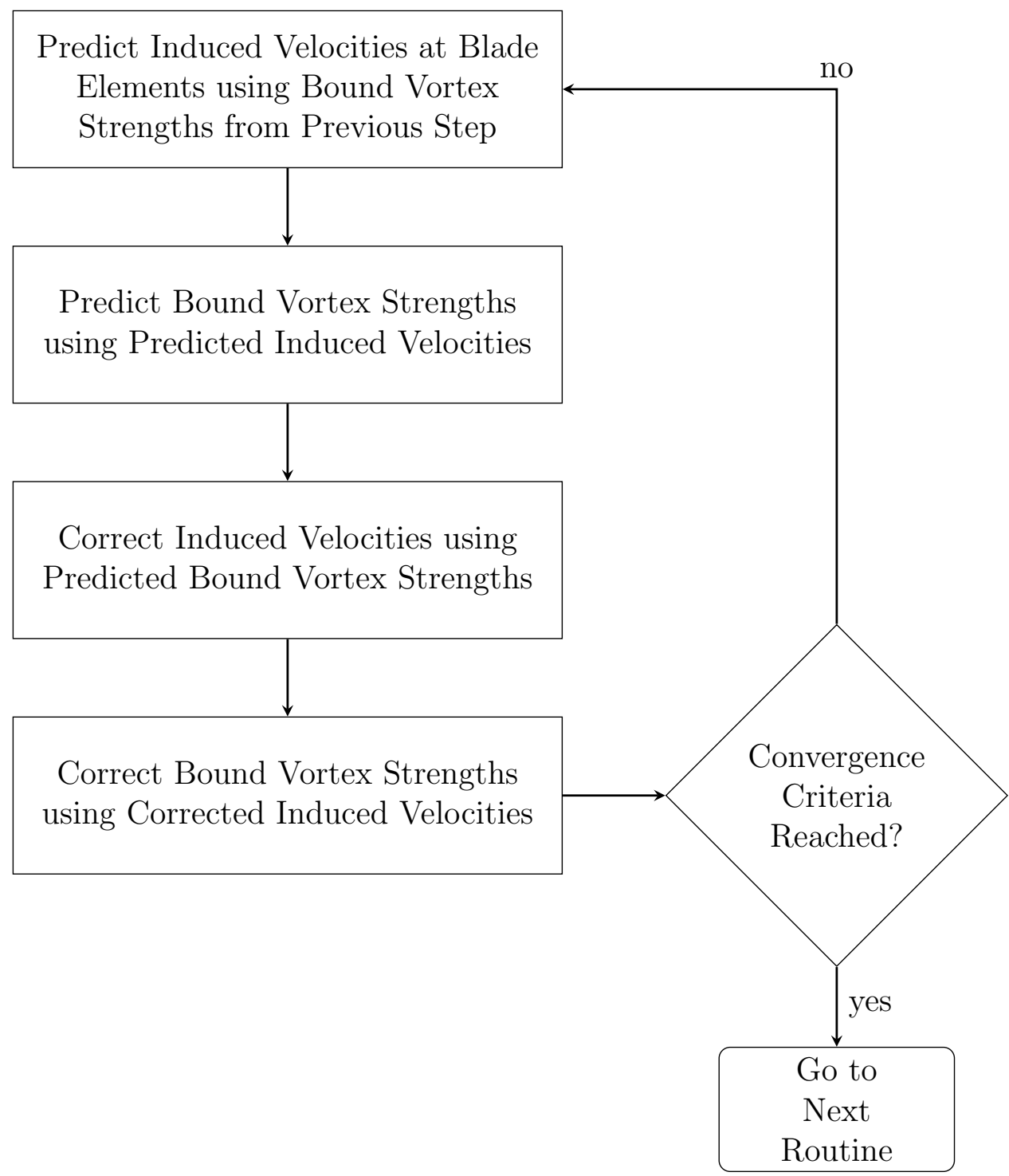

Figure 3.4: Bound vortex calculation using the predictor corrector method 


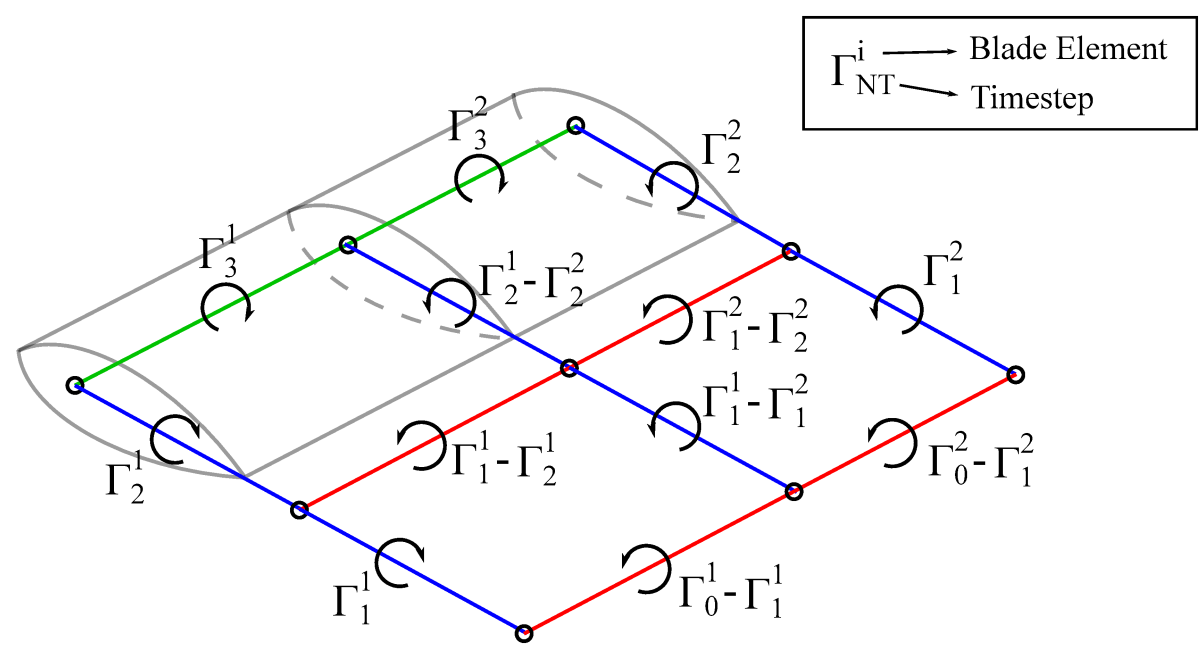

Figure 3.5: Schematic of the bound (Green), spanwise (Red) and trailing (Blue) vortices representing the wake behind a blade consisting of two elements after several timesteps. Adapted from Strickland et al. [41].

which are parallel to the span and chord of the blade, respectively. It should be noted that there are $n$ spanwise vortices and $n+1$ trailing vortices, where $n$ is the number of discretized sections along the blade. The strength of these vortex filaments are calculated based on the Kelvin's theorem for which any change in the bound vortex strength over time must be accompanied by an equal and opposite vortex strength in the wake [70], given by:

$$
\frac{D \Gamma_{B}}{D t}=0
$$

In order to satisfy the Kelvin's theorem, the spanwise vortex strength of the element $i$ for timestep $(N T-1)$ is given by

$$
\Gamma_{S(N T-1)}^{i}=\Gamma_{B(N T-1)}^{i}-\Gamma_{B(N T)}^{i}
$$


On the other hand, the strength of the trailing vortex filaments, at both end sections of the element is dictated by the Helmholtz Theorem and is equal to the strength of the bound vortex of that element. Therefore, the difference between the strengths of the bound vortices associated to two consecutive blade elements, results in the trailing vortex. The strength of the trailing vortex of element end $j$ for timestep $(N T-1)$ is given as

$$
\Gamma_{T(N T-1)}^{j}=\Gamma_{B(N T-1)}^{i}-\Gamma_{B(N T-1)}^{i-1}
$$

In the absence of a subsequent section, namely at the blade tip, the strength of the tip vortex is assumed to have the same magnitude and opposite direction as the bound vortex closest to the tip, given by:

$$
\Gamma_{T(N T-1)}^{j}=-\Gamma_{B(N T-1)}^{i}
$$

\subsection{Wake Convection}

Each filament vortex contains two points, one at the head and another at the tail, which are known as Lagrangian markers. Each marker (or node) in the wake convects with a local velocity. As the filaments first leaves the blade, its initial velocity is assumed to be equal to the sum of the induced velocities at the blade element end and the oncoming freestream velocity. Therefore the initial distance travelled by each node of the filament vortex is given by:

$$
\begin{aligned}
& \Delta x=\left(U_{t}+V_{o}\right) \Delta t \\
& \Delta y=V_{t} \Delta t \\
& \Delta z=W_{t} \Delta t
\end{aligned}
$$


where $\Delta t$ is the time increment and $U_{t}, V_{t}$, and $W_{t}$ are the induced velocities in the $x, y$, and $z$ directions at time $t$. In order to calculate the distance travelled by any node in the wake for a given time step, a second order Adams-Bashforth explicit integration formula is used. The predicted distance travelled is given as follows:

$$
\begin{aligned}
& \Delta x=\left(1.5 U_{t}-0.5 U_{t-\Delta t}+V_{o}\right) \Delta t \\
& \Delta y=\left(1.5 V_{t}-0.5 V_{t-\Delta t}\right) \Delta t \\
& \Delta z=\left(1.5 W_{t}-0.5 W_{t-\Delta t}\right) \Delta t
\end{aligned}
$$

It should be noted that the two markers, which constitute a vortex filament, move independently of each other. Therefore, each vortex filament is allowed to stretch and rotate while being convected in the flow field.

\subsection{Induced Velocity}

The induced velocity $\mathbf{V}_{\text {ind }}$ at a point $P$, located at position $\mathbf{r}$ relative to the filament vortex of infinitesimal length $d \mathbf{l}$ of strength $\Gamma_{v}$, can be evaluated using the well-known Biot-Savart law, written as:

$$
\begin{aligned}
\mathbf{V}_{\text {ind }} & =\frac{\Gamma_{v}}{4 \pi} \int \frac{\mathbf{r} \times d \mathbf{l}}{|\mathbf{r}|^{3}} \\
& =\frac{\Gamma_{v}}{4 \pi h}\left(\cos \beta_{1}-\cos \beta_{2}\right) \mathbf{e}
\end{aligned}
$$

where the angles $\beta_{1}$ and $\beta_{2}$ are called the view angles, the unit vector $\mathbf{e}$ is the direction vector of the induced velocity and $h$ is the perpendicular distance between the filament vortex and evaluation point $P$. Figure 3.6 shows a representation of the vortex filament for a point of interest. The reader can consult Refs. $[69,70,75]$ for the derivation of the Biot-Savart law. 


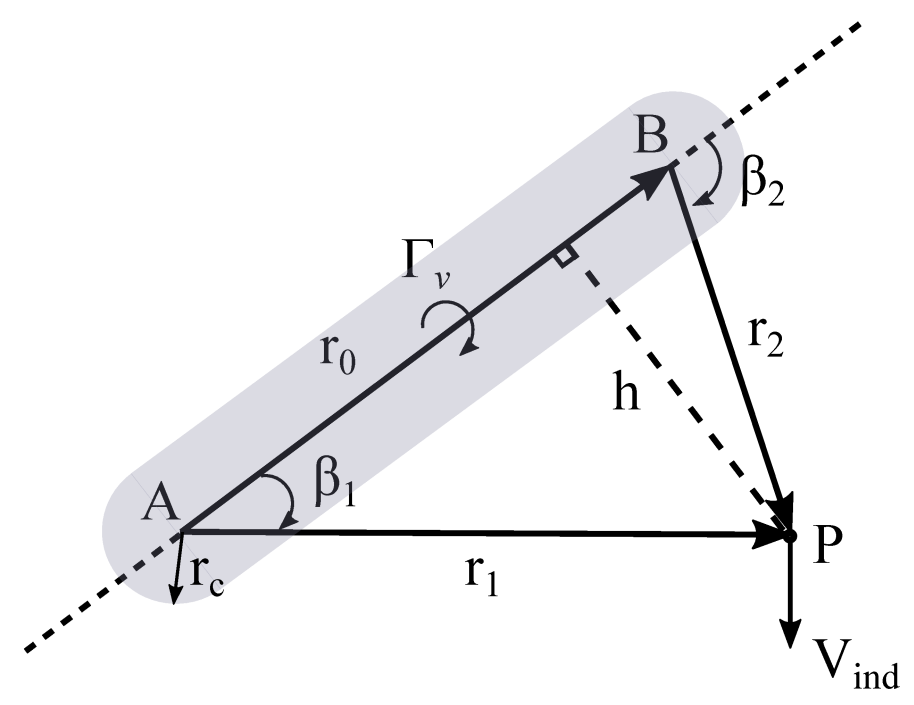

Figure 3.6: Velocity induced by a straight filament vortex of strength $\Gamma_{v}$ with vortex core representation. Adapted from Ref. [76]

For a straight filament vortex illustrated in Figure 3.6, $\mathbf{r}_{0}, \mathbf{r}_{1}$, and $\mathbf{r}_{2}$ represent the direction vectors $\mathrm{AB}, \mathrm{AP}$, and BP, respectively. A simplified form of Equation 3.24 can be derived by applying trigonometric relations, giving:

$$
\mathbf{V}_{\text {ind }}=\frac{\Gamma_{v}}{4 \pi} \frac{\mathbf{r}_{1} \times \mathbf{r}_{2}}{\left|\mathbf{r}_{1} \times \mathbf{r}_{2}\right|^{2}}\left(\frac{\mathbf{r}_{0} \cdot \mathbf{r}_{1}}{\left|\mathbf{r}_{1}\right|}-\frac{\mathbf{r}_{0} \cdot \mathbf{r}_{2}}{\left|\mathbf{r}_{2}\right|}\right)
$$

Although this equation is commonly found in textbooks [70,75], a singular solution is obtained whenever $\mathbf{r}_{1}$ and $\mathbf{r}_{2}$ are collinear [77]. The latter can be eliminated by removing the $\left(\mathbf{r}_{1} \times \mathbf{r}_{2}\right)$ term from the denominator, giving:

$$
\mathbf{V}_{\text {ind }}=\frac{\Gamma_{v}}{4 \pi} \frac{\left(\left|\mathbf{r}_{1}\right|+\left|\mathbf{r}_{2}\right|\right)\left(\mathbf{r}_{1} \times \mathbf{r}_{2}\right)}{\left|\mathbf{r}_{1}\right|\left|\mathbf{r}_{2}\right|\left(\left|\mathbf{r}_{1}\right|\left|\mathbf{r}_{2}\right|+\mathbf{r}_{1} \cdot \mathbf{r}_{2}\right)}
$$

Moreover, as $\left(\mathbf{r}_{1} \cdot \mathbf{r}_{2}\right)$ approaches zero (i.e., the angle between $\mathbf{r}_{1}$ and $\mathbf{r}_{2}$ is $\pm \pi$ ), Equation 3.26 shows a singular behaviour. In other words, as the evaluation point is approaching the vortex core which is represented by a cylinder and two spherical 


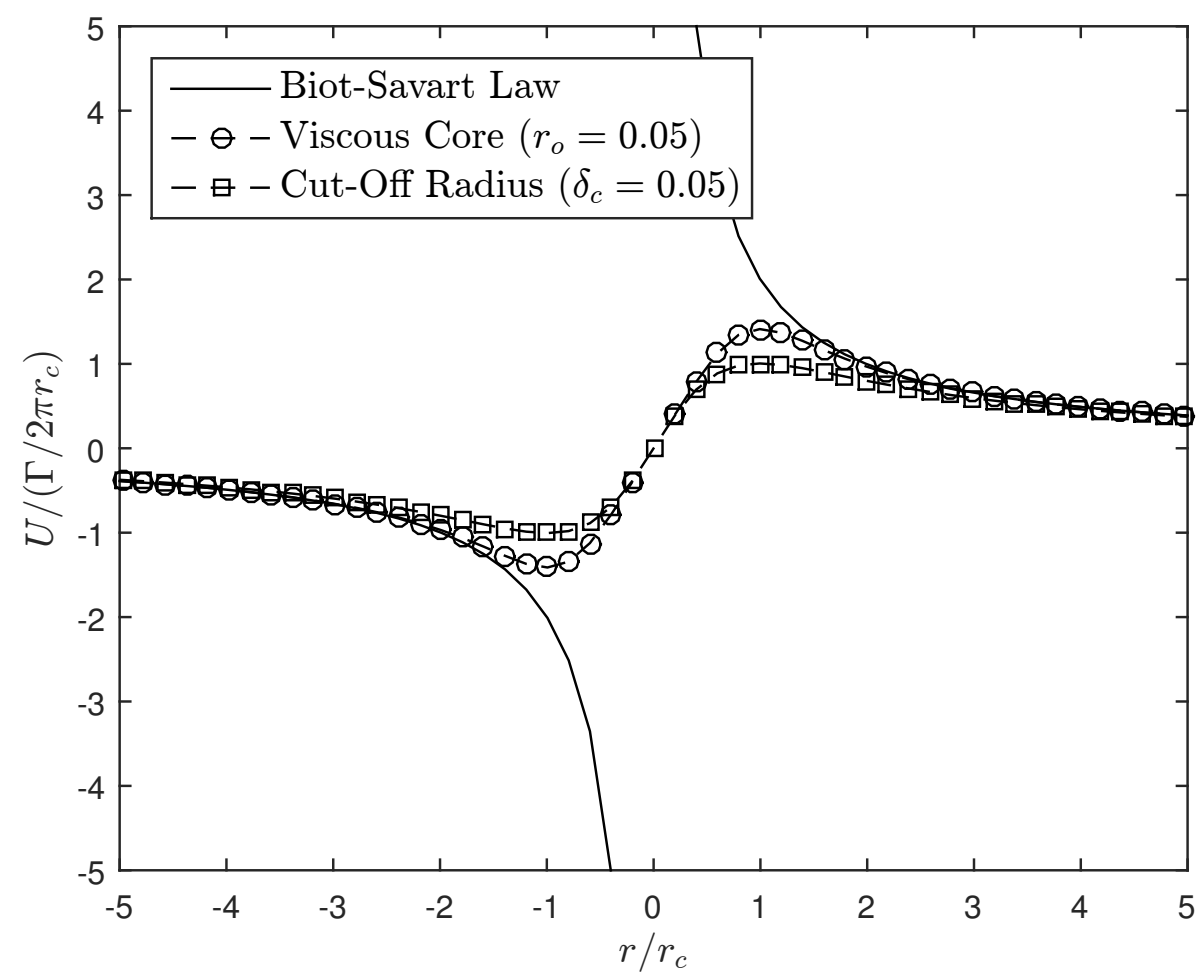

Figure 3.7: Induced velocity of a filament vortex evaluated using (i) Biot-Savart Law, (ii) viscous core model, and (iii) cut-off radius method, as a function of dimensionless distance from the vortex core $r / r_{c}$

caps with a core radius $r_{c}$ in Figure 3.6, the induced velocity experienced increases exponentially. This nonphysical behaviour can be removed with the introduction of a cut-off radius $[32,69]$ or viscous core model $[78,79]$ that mimic the viscous behaviour of a real vortex. Figure 3.7 illustrates the induced velocity distribution as a function of the distance $r$ from the point of interest to the vortex core.

The Biot-Savart law which is desingularized using the cut-off radius can be written as

$$
\mathbf{V}_{\text {ind }}=\frac{\Gamma_{v}}{4 \pi} \frac{\left(\left|\mathbf{r}_{1}\right|+\left|\mathbf{r}_{2}\right|\right)\left(\mathbf{r}_{1} \times \mathbf{r}_{2}\right)}{\left|\mathbf{r}_{1}\right|\left|\mathbf{r}_{2}\right|\left(\left|\mathbf{r}_{1}\right|\left|\mathbf{r}_{2}\right|+\mathbf{r}_{1} \cdot \mathbf{r}_{2}\right)+\left(\delta_{c}\left|\mathbf{r}_{0}\right|\right)^{2}}
$$


where the cut-off radius parameter $\delta_{c}$ defines the percentage of the vortex length $\mathbf{r}_{0}$ which represents vortex core. Within this region, the induced velocity smoothly decreases to zero as it approaches the vortex line. On the other hand, the viscous core method, defined in Equation 3.28, uses a factor $K$ which desingularizes the BiotSavart equation as $r$ tends to zero and prevents a high induced velocity in the vicinity region of the vortex core radius [37].

$$
\mathbf{V}_{\text {ind }}=K \frac{\Gamma_{v}}{4 \pi} \frac{\left(\left|\mathbf{r}_{1}\right|+\left|\mathbf{r}_{2}\right|\right)\left(\mathbf{r}_{1} \times \mathbf{r}_{2}\right)}{\left|\mathbf{r}_{1}\right|\left|\mathbf{r}_{2}\right|\left(\left|\mathbf{r}_{1}\right|\left|\mathbf{r}_{2}\right|+\mathbf{r}_{1} \cdot \mathbf{r}_{2}\right)}
$$

where

$$
K=\frac{r^{2}}{\left(r_{c}^{2 n}+r^{2 n}\right)^{1 / n}}
$$

The traditional core regularization (i.e., $r=h$ ) was preferred to the recent improved correction proposed by van Hoydonck et al. (2014) [76]. Although the latter method showed better agreement with the analytical approximation of the induced velocity produced by a thin-cored vortex ring (formulation not shown here), van Hoydonck et al.'s approach works only for very small discretizations, i.e., for $\Delta \theta<r_{c} / R \approx 10^{-5}$ where $\Delta \theta$ is the azimuthal discretization for the vortex ring. Figure 3.8 illustrates the induced velocity of a vortex ring discretized into small straightline vortex elements as a function of the core radius. The reader is referred to Saffman (1992) [69] for a detailed description of the analytical approximation of an inviscid vortex ring.

Furthermore, the accuracy of the simulation is highly dependent on the viscous core model used since the induced velocity field has a significant effect on the wake geometry and aerodynamic loads. The induced velocity can be represented using either a constant vortex model based on a desingularized algebraic profile such as Rankine $(n \rightarrow \infty)$ [81], Scully $(n=1)$ [82] and Vatistas $(n=2)$ [83] models or 


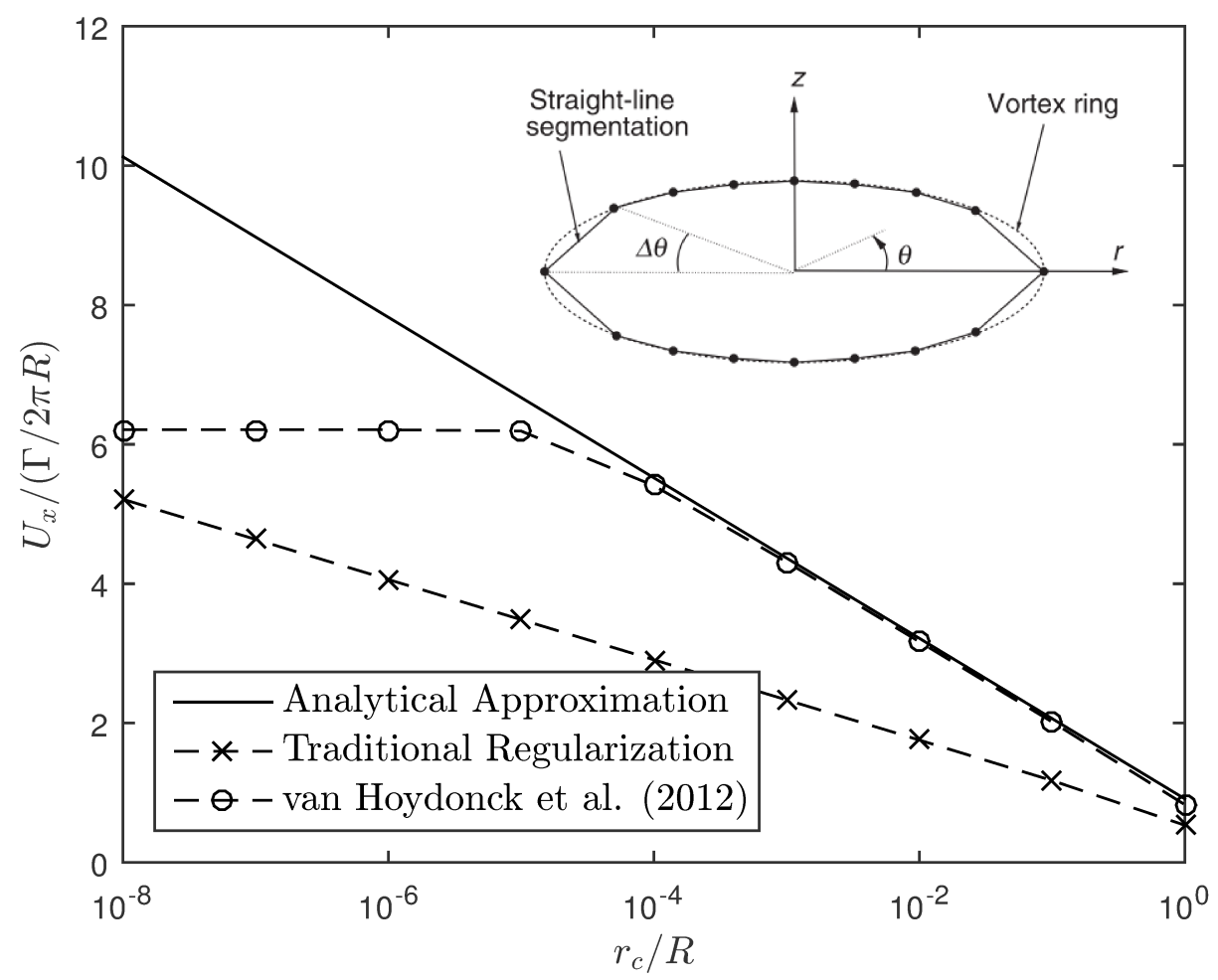

Figure 3.8: Axial induced velocity vortex ring comparison between (i) analytical approximation, (ii) traditional core regularization, and (iii) modified core regularization by van Hoydonck et al. [76], as a function of relative core size $r_{c} / R$. Adapted from Ref. [80]

a diffusive vortex model where the core radius grows with time according to the Lamb-Oseen's model [79]. The latter model, which is employed in this work, can be approximated using the Vatistas vortex model $(n=2)$.

The viscous core radius $r_{c}$ defined as the radial position around the filament vortex where the swirl velocity is maximum, was found to grow with time as

$$
r_{c}(t)=\sqrt{4 \alpha \nu t}
$$

where $\alpha=1.25643, \nu$ is the fluid viscosity and $t$ is the age of the vortex. However, at $t=0$, the swirl velocity given by Equation 3.30 is singular. To remove this singularity, 
the filament vortex can be assumed to have a finite core size $\left(r_{o}\right)$ at its origin $(t=0)$. The vortex core growth which is a modified Lamb-Oseen model proposed by Squire in 1954 and employed in this work, is given by Equation 3.31. This model includes an average apparent or "eddy" viscosity $(\delta \nu)$ to account for the effects of turbulence generation on the enhanced diffusion of vorticity $[79,84]$.

$$
r_{c}(t)=\sqrt{r_{o}^{2}+4 \alpha \delta \nu t}
$$

Typical initial core radius of wing tip vortices was measured to be $5-10 \%$ chord length by Bhagwat and Leishman (2002) [79]. In this current work, $r_{o}$ is assumed to be a percentage of the chord length and blade radius when evaluating the bound vortex strength and shed vortices, respectively. The eddy viscosity coefficient $\delta$ was found to be dependent on the vortex Reynolds number $\left(R e_{v}=\Gamma_{v} / \nu\right)$, given by

$$
\delta=1+a_{1}\left(\frac{\Gamma_{v}}{\nu}\right)
$$

where $a_{1}$ is in the order of $10^{-4}$ based on the experiments performed by Bhagwat and Leishman $[79,85]$.

\subsection{Stall Delay Model}

The effect of rotation affects the boundary layer on rotor blades at the onset of stall. These rotational effects are induced by a phenomenon referred to as rotational augmentation which delay the separation point at higher angle of attack as compared to two-dimensional $(2 D)$ case. In the 1940's, Himmelskamp [86] was the first to quantitatively analyze the three-dimensional (3D) effect on propeller blades and confirmed that rotation does delay the laminar separation point. Since then, various 
rotational effects or 'stall delay' models have been formulated by Snel et al. [87], Du \& Selig [88], Chaviaropoulos \& Hansen [89], Lindenburg [90], and Bak et al. [91], to name a few. These models correct the non-rotating lift $\left(C_{L, 2 D}\right)$ coefficients with an increment $\left(\Delta C_{L}\right)$. The rotational effect on $2 \mathrm{D}$ drag coefficient $\left(C_{D, 2 D}\right)$, however, is not clearly understood [92]. Lindenburg [93] predicts an increase in drag since energy is required for 'centrifugal pumping' due to the radial flows which exist in rotating blades under stall conditions. Most 3D correction models predict an increment in drag $[89,91,93]$ which correspond to the measurements on the NREL Phase VI experiment, showing a drastic increase in the aerodynamic drag coefficient $[67,92,94]$. However, some models describe a reduction [88] or no influence at all [87] on the drag coefficient.

Equations 3.33 and 3.34 describe the methodology used in most stall delay models

$$
\begin{gathered}
C_{L, 3 D}=C_{L, 2 D}+f_{L} \Delta C_{L} \\
C_{D, 3 D}=C_{D, 2 D}+f_{D} \Delta C_{D}
\end{gathered}
$$

where the subscripts $2 D$ and $3 D$ refer to non-rotating and rotating characteristics, respectively. $\Delta C_{L}$ and $\Delta C_{D}$ are the difference between the $C_{L, 2 D}$ and $C_{D, 2 D}$ wind tunnel measurements and the $2 \mathrm{D} C_{L}$ and $C_{D}$ if hypothetically the flow would remained attached at all angle of attacks [91]. In other words, $\Delta C_{L}$ is the difference between $C_{L, 2 D}$ and the inviscid $C_{L}\left(C_{L, i n v}=2 \pi\left(\alpha-\alpha_{0}\right)\right.$ where $\alpha_{0}$ is the zero-lift angle) and $\Delta C_{D}$ is the difference between the $C_{D, 2 D}$ and the drag coefficient at zero angle of attack, $C_{D, 0}[88-90,95]$. Sometimes, the extended linear lift $\left(C_{L, \text { lin }}\right)$ is used in place of the $C_{L, i n v}[96]$. This method was employed in this code as it is believed to provide a better representation (extrapolation) of the airfoil characteristic. $f_{L}$ and $f_{D}$ are 
Table 3.1: Summary of functions used in stall delay models

\begin{tabular}{|c|c|}
\hline Model & Functions \\
\hline Snel et al. [87] & $f_{L}=3\left(\frac{c}{r}\right)^{2}$ \\
\hline \multirow[t]{4}{*}{ Du \& Selig [88] } & $f_{L}=\frac{1}{2 \pi}\left[\frac{1.6(c / r)}{0.1267} \frac{a-(c / r)^{\frac{d}{\lambda} \frac{R}{r}}}{b+(c / r)^{\frac{d}{\lambda}} \frac{R}{r}}-1\right]$ \\
\hline & $f_{D}=-\frac{1}{2 \pi}\left[\frac{1.6(c / r)}{0.1267} \frac{a-(c / r)^{\frac{d}{2 \lambda} \frac{R}{r}}}{b+(c / r)^{\frac{d}{2 \lambda} \frac{R}{r}}}-1\right]$ \\
\hline & where $a=b=d=1$, \\
\hline & and $\lambda=\frac{\omega R}{\sqrt{V_{o}^{2}+(\omega R)^{2}}}$ \\
\hline Chaviaropoulos \& Hansen [89] & $f_{L}=f_{D}=2.2\left(\frac{c}{r}\right) \cos ^{4} \beta$ \\
\hline Lindenburg [90] & $f_{L}=3.1\left(\frac{\omega r}{V_{r e l}}\right)^{2}\left(\frac{c}{r}\right)^{2}$ \\
\hline
\end{tabular}

functions used in the specific stall delay models and are briefly summarized in Table 3.1. It should be noted that the lift and drag corrections were applied up to $30^{\circ}$ angle of attack, after which the correction was linearly decreased to zero at an angle of attack of $50^{\circ}[89,90,97]$.

The stall delay models previously described do not in fact correct for blade-specific and solution dependent effects since sectional airfoil data are corrected prior to the actual simulation. Other models proposed by Bak et al. [91] and Dowler \& Schmitz [95] provide promising attempt to include these effects (not shown here). Figure 3.9 shows various stall delay models used to correct the $2 \mathrm{D}$ wind tunnel lift and drag coefficients for an S809 airfoil. Since modeling stall delay is not the focus of the current study, only the contemporary stall delay models such as Snel et al. and Du \& Selig to correct for rotational effects, are employed. 

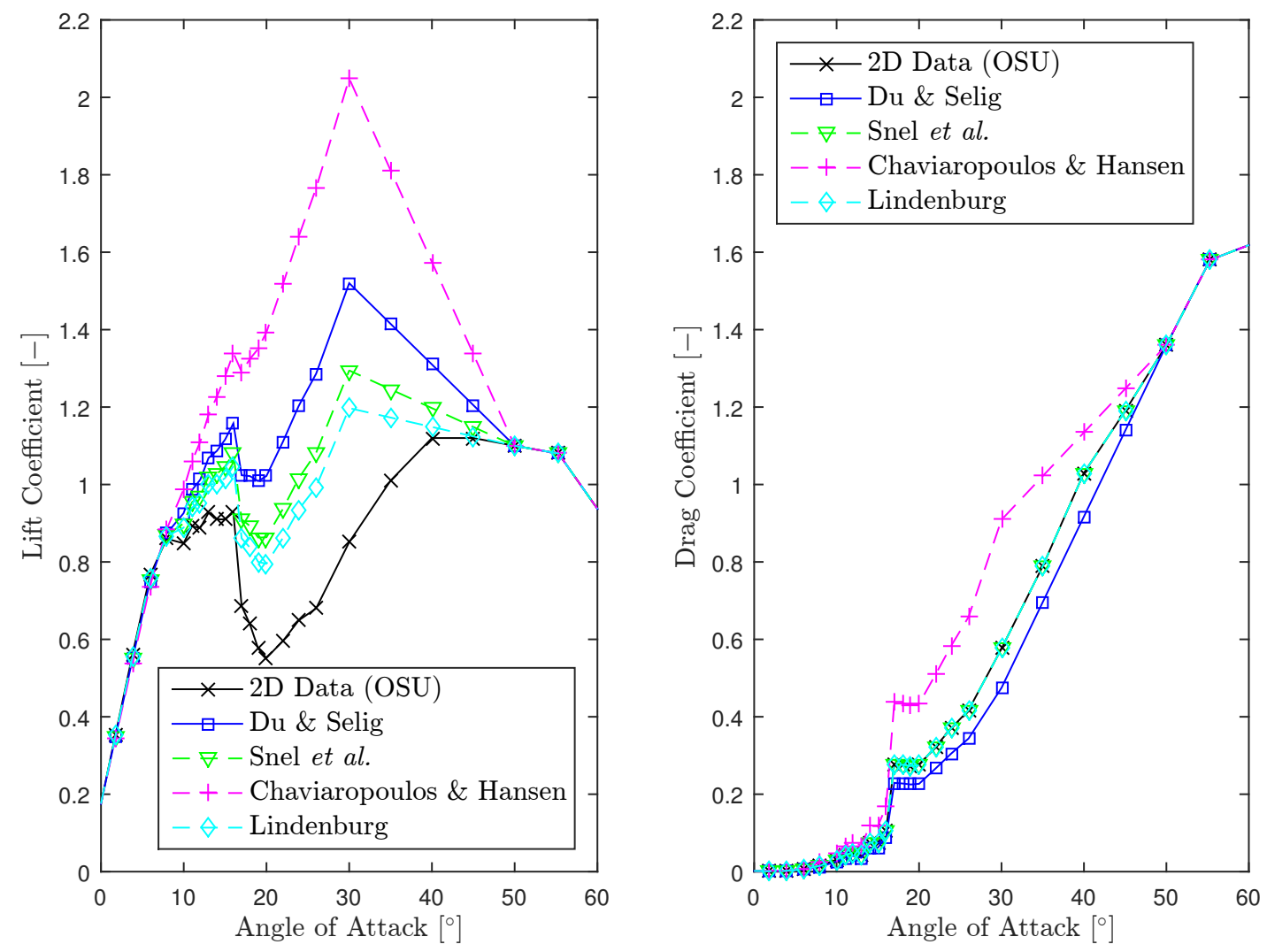

Figure 3.9: Effect of various 3D corrections applied to the wind tunnel (2D) data from OSU [47] for the S809 airfoil located at $46.6 \% \mathrm{R}$ along a blade rotating at 7.5 $\mathrm{rad} / \mathrm{s}$ and $R e=6.5 \times 10^{5}$

\subsection{Blade loading and Rotor Performance}

Once the effect of the wake (i.e., the total induced velocity) is known, the loads acting in the tangential $\left(F_{t}\right)$ and normal $\left(F_{n}\right)$ direction on the each blade element can be determined as:

$$
\begin{gathered}
F_{t}=\frac{1}{2} \rho\left|\mathbf{V}_{r e l}\right|^{2} c C_{t} \\
F_{n}=\frac{1}{2} \rho\left|\mathbf{V}_{r e l}\right|^{2} c C_{n}
\end{gathered}
$$




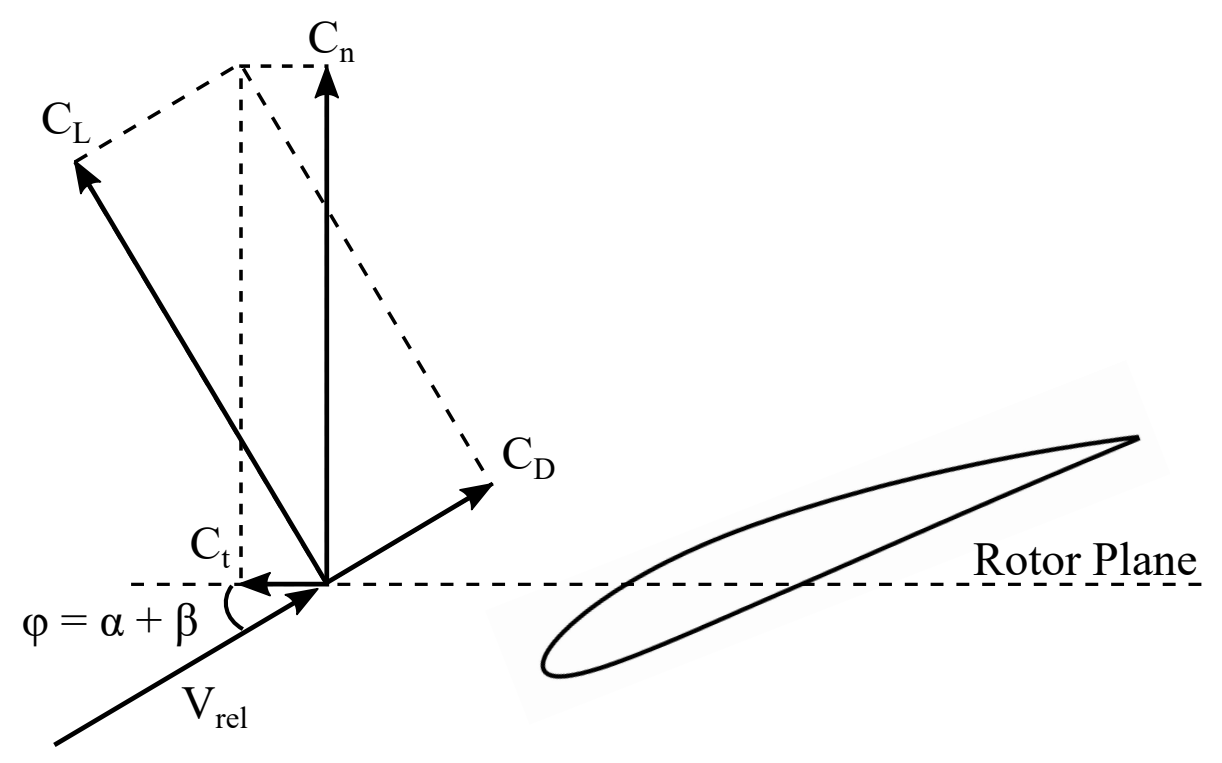

Figure 3.10: Load coefficients acting on blade element

where the normal $\left(C_{n}\right)$ and tangential $\left(C_{t}\right)$ coefficients are calculated using Equations 3.37 and 3.38 based on the airfoil coefficients for a specific angle of attack, as illustrated in Figure 3.10.

$$
\begin{gathered}
C_{n}=C_{L} \cos (\phi)+C_{D} \sin (\phi) \\
C_{t}=C_{L} \sin (\phi)-C_{D} \cos (\phi)
\end{gathered}
$$

where the flow angle, $\phi$ is the sum of the local angle of attack $(\alpha)$, twist and pitch $(\beta)$ acting on the blade element.

Once the normal and tangential force for each section have been determined, the torque $(Q)$ and axial thrust $(T)$ can be calculated by assuming linear variation along each element [11], giving the following. 


$$
\begin{aligned}
Q= & \sum_{i=2}^{N B(n-1)}\left[\frac{1}{3} \frac{\left(F_{t, i}-F_{t, i-1}\right)}{\left(r_{i}-r_{i-1}\right)}\left(r_{i}^{3}-r_{i-1}^{3}\right)\right. \\
& \left.+\frac{1}{2} \frac{\left(F_{t, i-1} r_{i}-F_{t, i} r_{i-1}\right)}{\left(r_{i}-r_{i-1}\right)}\left(r_{i}^{2}-r_{i-1}^{2}\right)\right] \\
T= & \sum_{i=2}^{N B(n-1)}\left[\frac{1}{2} \frac{\left(F_{n, i}-F_{n, i-1}\right)}{\left(r_{i}-r_{i-1}\right)}\left(r_{i}^{2}-r_{i-1}^{2}\right)\right. \\
& \left.+\frac{\left(F_{n, i-1} r_{i}-F_{n, i} r_{i-1}\right)}{\left(r_{i}-r_{i-1}\right)}\left(r_{i}-r_{i-1}\right)\right]
\end{aligned}
$$

where subscript $i$ denotes the element section and $n$ denotes the number of sections that discretized the blade. It should be noted that each element is bounded by two sections.

The aerodynamic power produced by the wind turbine can then be calculated as the product of the rotational velocity and the torque of the rotor. Furthermore, for comparison purposes, the coefficients of thrust $\left(C_{T}\right)$, torque $\left(C_{Q}\right)$, and power $\left(C_{P}\right)$ are calculated as described in Section 1.1. For completeness, the equations are repeated below. Also, note that for the purpose of this work, the rotor performance is averaged over 1 revolution.

$$
\begin{gathered}
C_{T}=\frac{T}{1 / 2 \rho A V_{o}^{2}} \\
C_{Q}=\frac{Q}{1 / 2 \rho R A V_{o}^{2}} \\
C_{P}=\frac{Q \omega}{1 / 2 \rho A V_{o}^{3}}
\end{gathered}
$$




\subsection{Parallel Implementation}

Although the vortex method employed in DR_HAWT is considerably less computationally expensive than a full CFD simulation, when parametric studies are conducted to investigate the effect of varying the geometry and flow conditions, the computational cost becomes more pronounced. Using similar implementation to Fereidooni's FEM-Vort [68], parallel capability was implemented into DR_HAWT.

Parallel computing takes advantage of dividing the computational workload amongst several processors thereby reducing the number of tasks each processor must accomplish. These distributed tasks are then solved simultaneously (in parallel) by each processor and the results are recombined in the shared memory. However, it should be noted that the current vortex model is not suited for distributed-memory machines. DR_HAWT implements parallel computing using OpenMP, a shared memory Fortran parallelization Application Programming Interface (API). By integrating "\$OMP" directive into the code, repeated calculations such as the induced velocity calculations, can be solved in parallel thereby decreasing the computational runtime.

To illustrate the capability of DR_HAWT to run simulations in parallel configuration, the MEXICO three-bladed wind turbine, where each blade was discretized into 21 sections, was simulated for 200 steps (4D wake lengths) at a wind velocity of $24 \mathrm{~m} / \mathrm{s}$ and a rotational velocity of $424.5 \mathrm{rpm}$. Figure 3.11 shows the speed-up ratio $S$, which is defined as $T_{1} / T_{N}$ where $T$ is the computational runtime and the subscript refers to the number of cores. All simulations investigated in this work, unless otherwise stated, were performed on the departmental fortran computer running Centos 6.5. This computer consists of 2 quad cores Intel (R) Xeon (R) CPU L5410 @ $2.33 \mathrm{GHz}$ with 16 GB ECC memory. The computational runtime for the aforementioned example was 3.5 hours in serial and 0.5 hour using 8 cores. As can be 


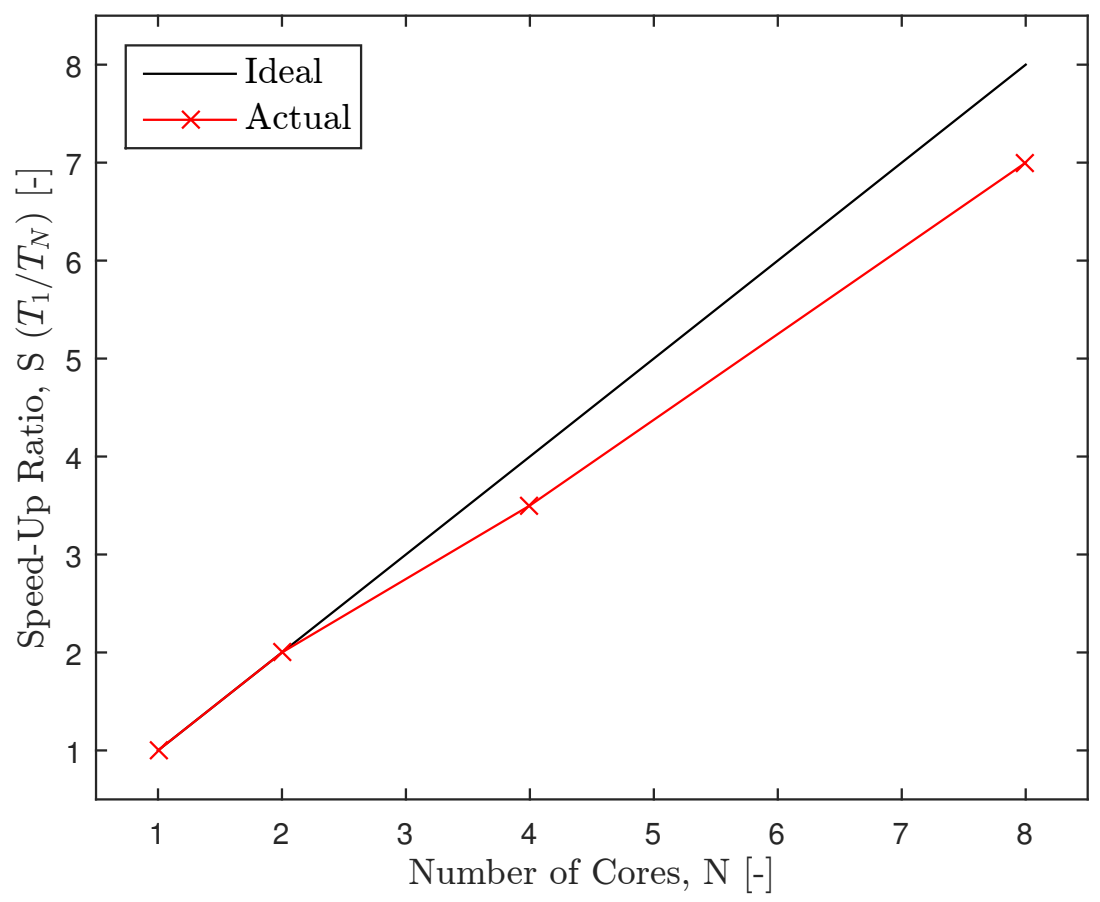

Figure 3.11: Parallel capability of DR_HAWT

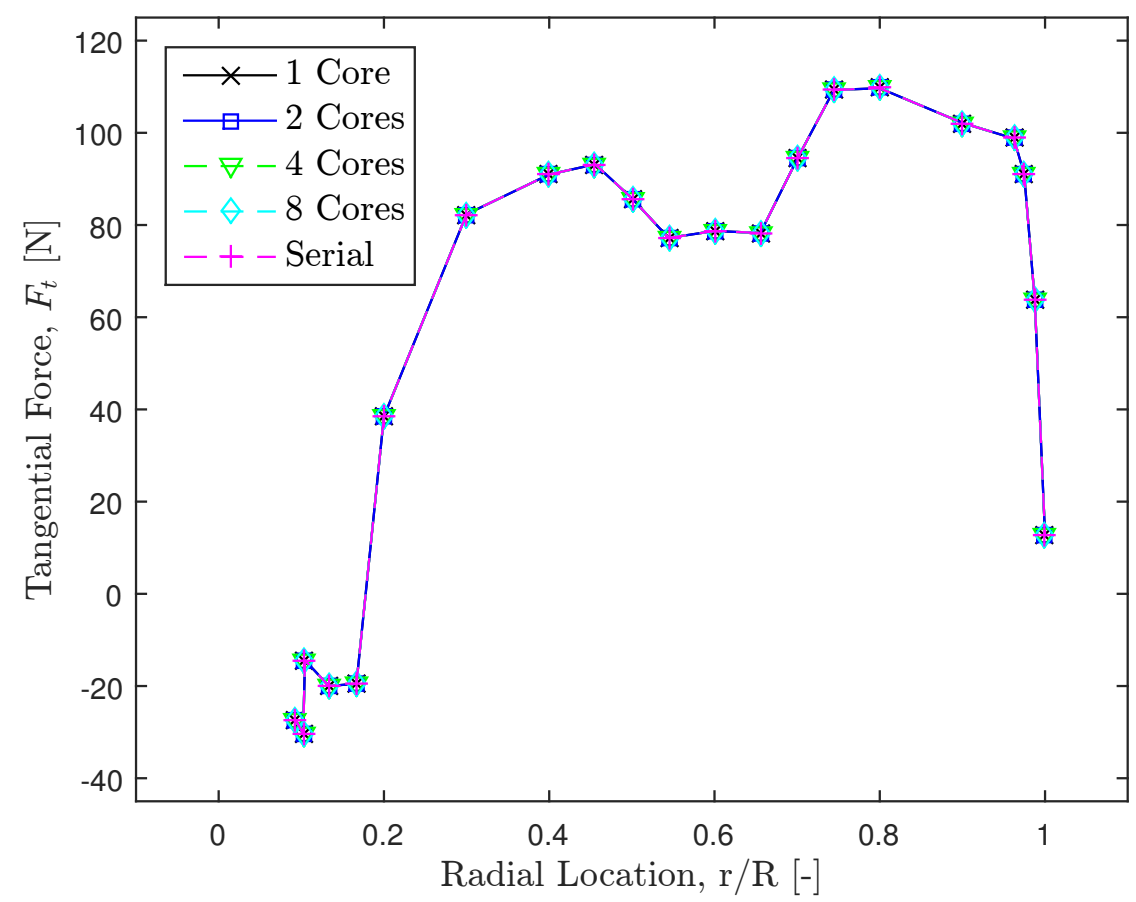

Figure 3.12: Verification of parallel implementation in DR_HAWT 
seen in Figure 3.11, DR_HAWT was about 90\% efficient when the maximum available number of cores (i.e., 8 cores) were used. In addition, to ensure that the parallel computing is implemented correctly, various outputs from the parallel simulations were compared to the serial results. Figure 3.12 illustrates the tangential force obtained from the study mentioned previously. The exact match between the results of the parallelized code for various number of cores and the serial code confirms that the parallel computing was correctly implemented in DR_HAWT.

\subsection{Program Structure}

To sum-up, the Dual-Rotor Horizontal-Axis Wind Turbine (DR_HAWT) code, implemented using FORTRAN 95, is capable of simulating a horizontal axis wind turbine in single or dual-rotor configuration. The general procedure used in DR_HAWT, as illustrated in Figure 3.13, is based on the code developed by Strickland et al. in 1980 .

The flow characteristics are input in terms of oncoming wind velocity, as well as the density and viscosity of the fluid. Due to the transient nature of the vortex filament method, the timestep increment in terms of seconds or degrees per step and the number of steps are also input. In addition, the user chooses the wind turbine configuration and the rotor geometry and specifications are extracted from the rotor input files accordingly. Based on the user input rotor configuration, the required blade matrices are created. The user can also choose the desired corrections such as rotational augmentation and vortex model, as well as the output parameters.

The bound vorticity at each blade section is assigned a zero value for the first iteration (or step). The coordinates of each blade element are determined and the induced velocity based on the bound vortex strength is computed for each element. For the first iteration, since the initial vorticity has been set to zero and no vortices 


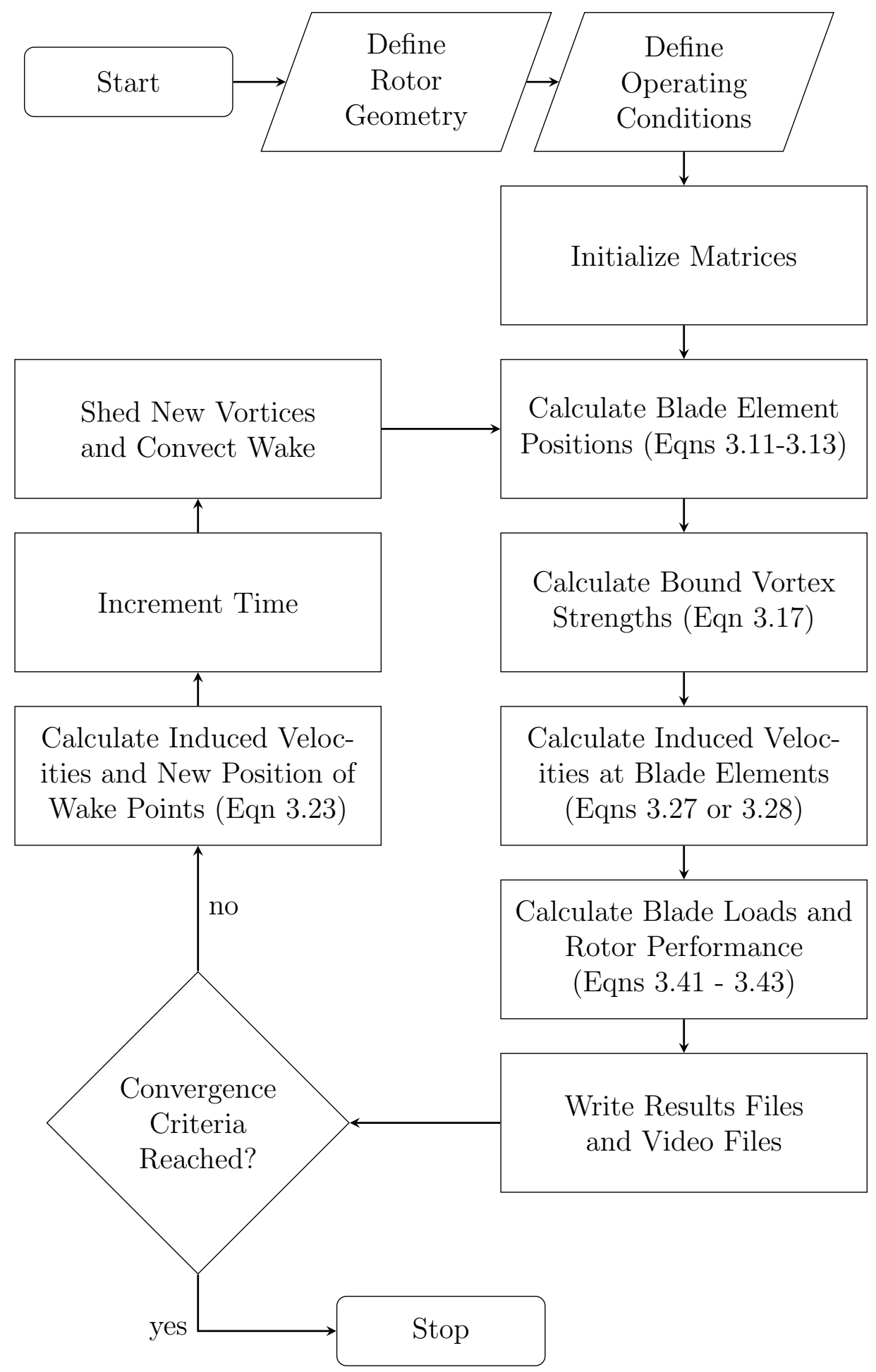

Figure 3.13: Program structure 
have previously been shed, the induced velocities have a zero value. To calculate the bound vorticities, a predictor corrector method is employed where the vortex strength is calculated based on the previous calculated induced velocities (see Section 3.3). This process is repeated until the differences between the predicted and corrected values are below $1 \times 10^{-3}$.

Once the bound vorticities and the induced velocities have been corrected, the tangential and normal forces at each blade element are computed. Applying the blade element theory (BET), the torque, thrust and power coefficients $\left(C_{Q}, C_{T}\right.$, and $C_{P}$, respectively) can be calculated for the current iteration. Next, a second order Adams-Bashforth explicit integration formula is used to calculate the new location of each lattice point. The timestep is then increased and the bound vortices are shed. For each iteration, the results files are updated and a new set of video files are created, if required. These video files, which are.VTK format, can then be opened in ParaView [98], an open-source data visualization and analysis tool which allows the user to animate the simulation. To illustrate DR_HAWT's ability to produce wake visualization, the NREL Phase VI two-bladed wind turbine was simulated. Figure 3.14 shows the wake structure for the Phase VI turbine.

This process is repeated for the number of desired steps or until the average $C_{P}$ value over a full rotation is below $1 \times 10^{-4}$ between two consecutive rotations. Furthermore, various error checking procedures are implemented in DR_HAWT to alert the user of any possible issues with the current configuration. 

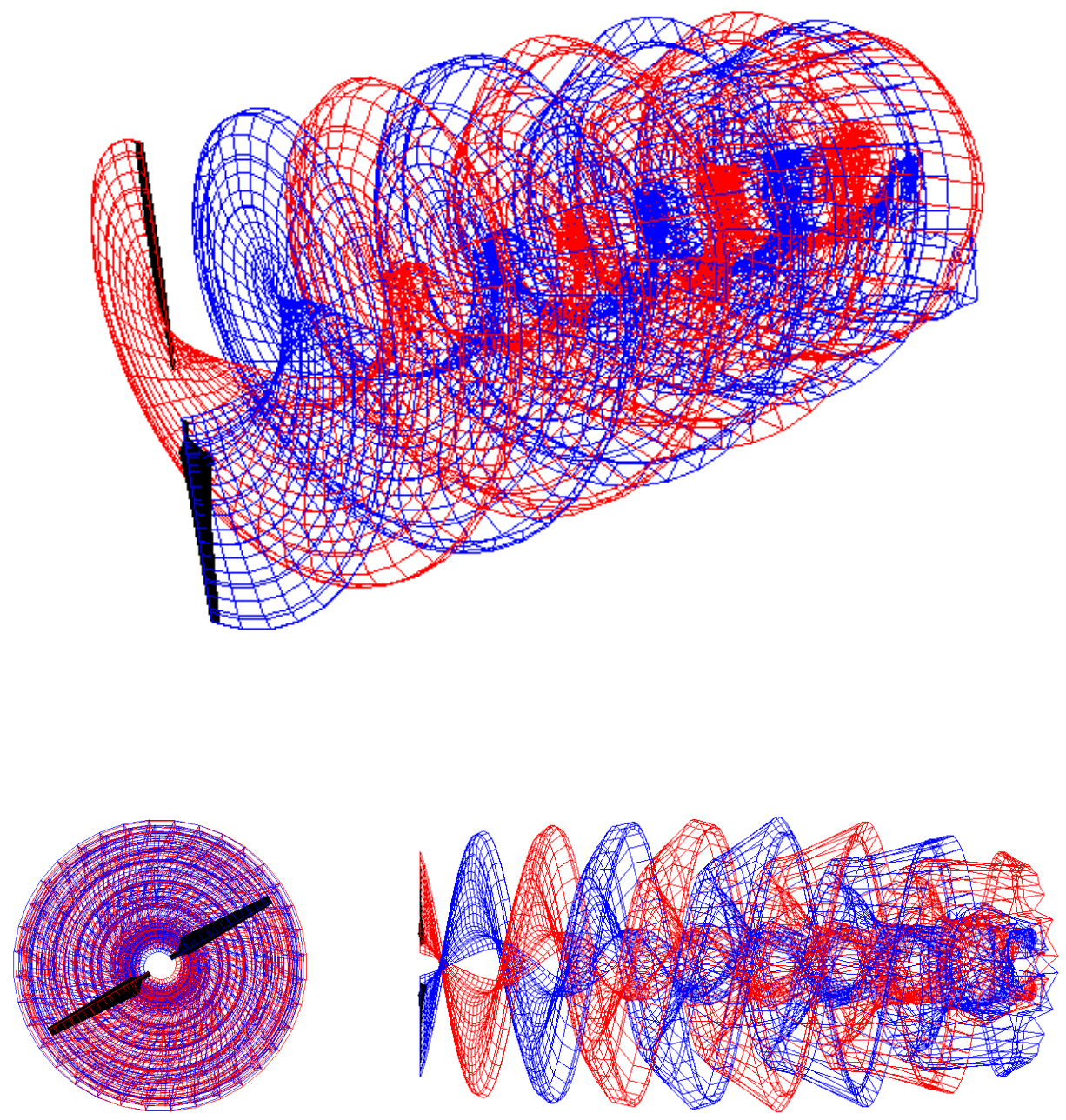

Figure 3.14: NREL Phase VI Wake visualization using ParaView where the wake from each blade is illustrated with a different color 


\section{Chapter 4}

\section{Verification and Validation Methodology}

To ensure that DR_HAWT was implemented properly, several verification and validation test cases were performed. As a first verification test, the accuracy of the computational solution is measured against the analytical solution for an elliptical planform wing with high aspect ratio. Subsequently, the effect on the downwind rotor from various oncoming vortices was analyzed to verify that the code responds correctly to a change in vortex strength and direction. Finally, to validate the single-rotor portion of the code, two well-known wind tunnel experiments - the National Renewable Energy Laboratory (NREL) Unsteady Aerodynamics Experiment (UAE) [47] and the Model Experiments in Controlled conditions (MEXICO) project [50]—were computed in DR_HAWT and compared to their measurements.

\subsection{Elliptic Wing}

The lift distribution on an elliptic planform wing with no twist causes the downwash to be a constant along the span. This constant induced downwash velocity $w(y)$ can be analytically evaluated by Equation 4.1. For a wing with non-elliptic planform, the local twist or camber along the span has to be adjusted to produce an elliptic loading 


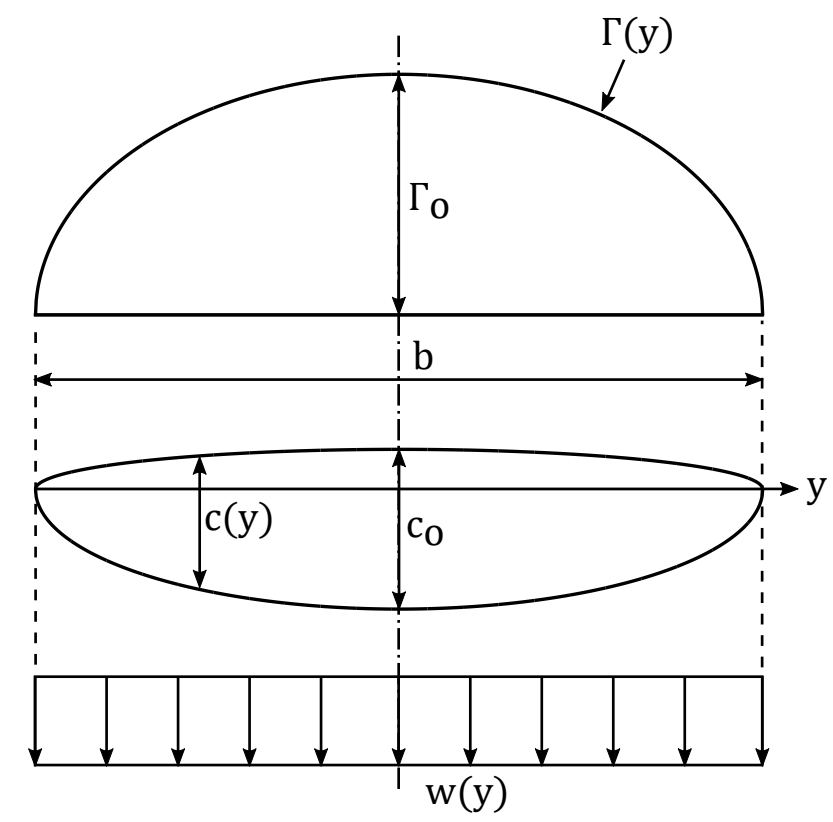

Figure 4.1: Chord and spanwise circulation distributions for a typical elliptical wing with low aspect ratio. Adapted from Bertin and Smith (1998) [75]

and therefore, constant downwash. Figure 4.1 illustrates a typical elliptic wing with span $b$, root chord $c_{o}\left(c_{o}=c(y=0)\right)$ and its corresponding circulation $\Gamma(y)$ and chord $c(y)$ distributions as a function of distance $y$ along the span, defined by Equations 4.2 and 4.3. The reader is referred to Refs. [75] and [70] for a detailed description of the elliptical lift distribution.

$$
\begin{gathered}
w(y)=-\frac{\Gamma_{o}}{2 b} \\
\Gamma(y)=\Gamma_{o}\left[1-\left(\frac{y}{b / 2}\right)^{2}\right]^{1 / 2} \\
c(y)=c_{o}\left[1-\left(\frac{y}{b / 2}\right)^{2}\right]^{1 / 2}
\end{gathered}
$$

where $\Gamma_{o}$ is maximum circulation $\left(\Gamma_{o}=\Gamma(y=0)\right)$ which is a function of the oncoming 
wind velocity $V_{o}$, the lift coefficient $C_{L}$, and the aspect ratio $R$ (commonly defined as $R=b^{2} / S$ where $\left.S=\pi b c_{o} / 4\right)$, given by the following equation.

$$
\Gamma_{o}=\frac{2 b V_{o} C_{L}}{\pi R}
$$

Figure 4.2 shows the analytical and computed downwash of an untwisted elliptic wing having a wing span of $10 \mathrm{~m}$, a root chord of $0.1 \mathrm{~m}$, and a constant $5^{\circ}$ angle of attack. The wing consists of constant airfoil sections with lift coefficients based on thin airfoil theory (i.e., $C_{L}=2 \pi\left(\alpha-\alpha_{o}\right)$ where $\alpha_{o}$ is the angle of attack at $\left.C_{L}=0\right)$. To mimic a fixed-wing case in DR_HAWT, a single-bladed rotor with the elliptic geometry defined by Equation 4.3 is placed in a flow field where the oncoming wind velocity is $10 \mathrm{~m} / \mathrm{s}$ and a zero angular velocity. In addition, the blade was pitched $85^{\circ}$ from the rotor plane to produce an angle of attack of $5^{\circ}$.

The analytical downwash for the elliptic wing is compared with three computed results in which the discretized elements were varied from 25 elements equally-spaced to 50 elements equally-spaced to 25 elements distributed using a full-cosine discretization scheme (see Appendix A) over the span of the blade. As can be seen in Figure 4.2, the downwash computed using DR_HAWT shows good agreement as compared to the analytical solution with the exception of the wing tips. However, the computed results tend to provide better agreement as the number of elements increased near the tip region. The discrepancy at the tips of the blade is also due to the fact that the analytical solution assumes a rigidly flat wake $[32,34,70,75]$ whereas the wake in DR_HAWT is free to evolve in three-dimensional (3D) space which results in a significant effect from the tip vortices. 


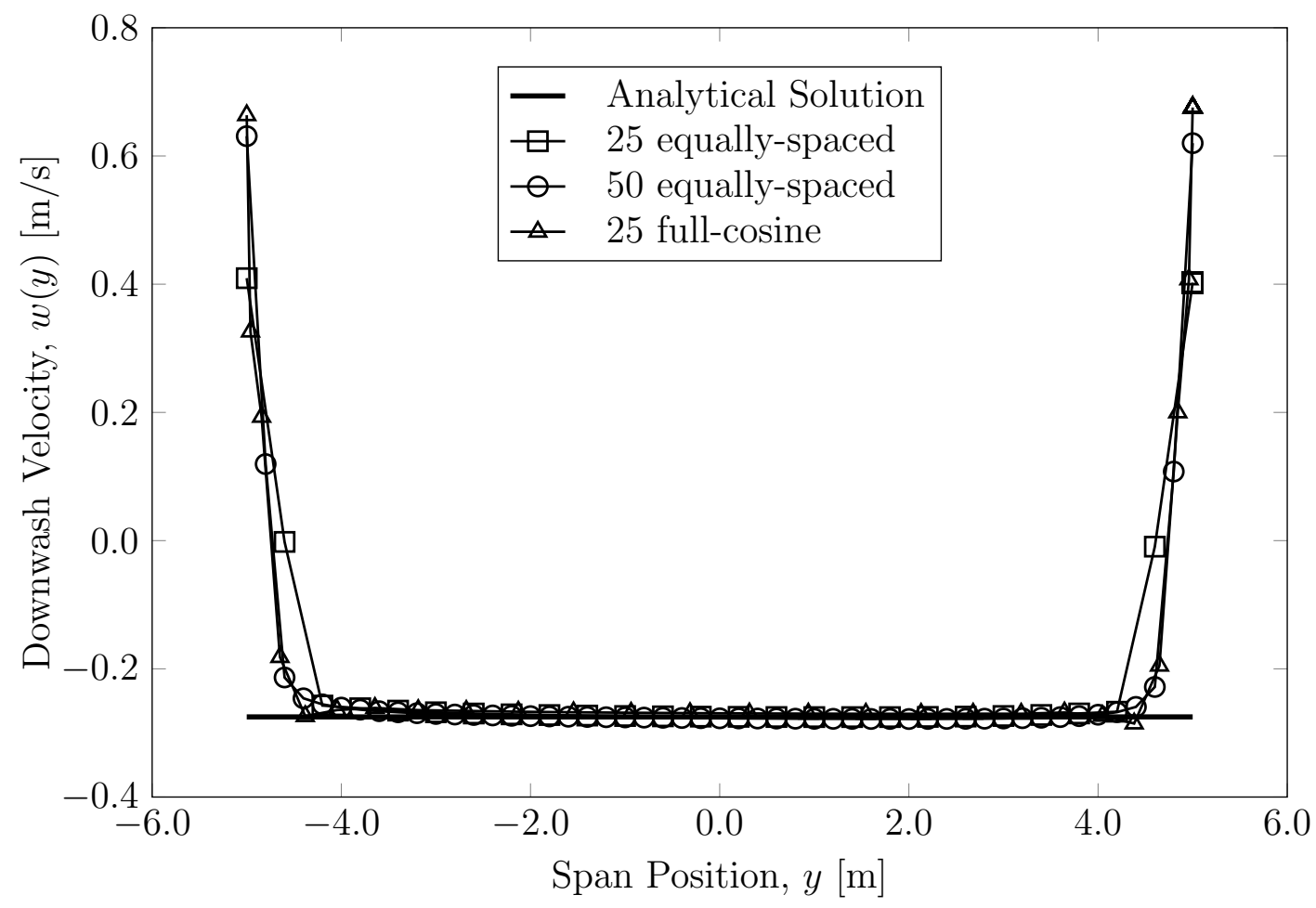

Figure 4.2: Comparison between computed and analytical downwash distribution over an elliptic wing with high aspect ratio. The three simulated wings consist of 25 elements equally-spaced, 50 elements equally-spaced, and 25 elements distributed using a full-cosine discretization scheme.

\subsection{Blade-Vortex Interaction}

Blade-Vortex Interaction (BVI) is the phenomenon where a vortex with prescribed strength, either fixed at a location or convecting with the free-stream interacts with the flow field of a blade. The axis of the vortex can either be parallel or perpendicular to the chord of the downwind blade. Figure 4.3 illustrates the span (starting vortex) and trailing (tip vortex) vortices approaching a blade element. As a span vortex approaches a blade, a downwash is induced on the blade which decreases the angle of attack, thereby decreasing lift. This induced velocity changes direction as the vortex passes the leading edge of the blade. On the other hand, an approaching 
a) Starting Vortex

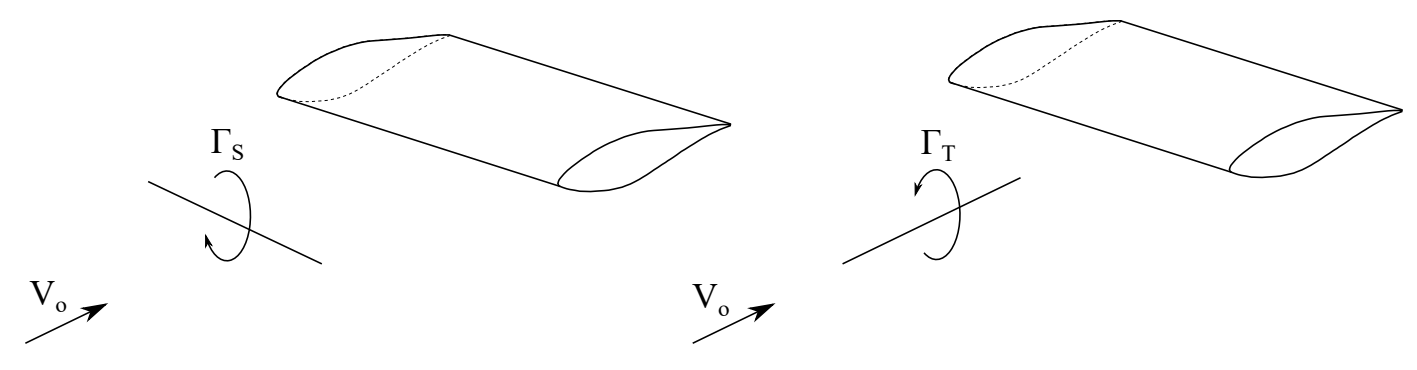

Figure 4.3: Schematic of a blade-vortex interaction phenomenon

trailing vortex tends to induce a downwash on the blade element. Furthermore, this downwash is expected to peak as the vortex passes the leading edge of the blade element. Although this work does not focus on BVI, it is important to verify that DR_HAWT accounts for these interactions correctly.

To simulate the effect of an approaching vortex in DR_HAWT, two stationary single-bladed rotors pitched at $90^{\circ}$ which induces a zero angle of attack on the blade, were modeled with the influence of an oncoming wind velocity of $10 \mathrm{~m} / \mathrm{s}$. The downwind blade is placed $5 \mathrm{~m}$ downstream of the upwind blade which is equivalent to approximately 20 timesteps. Furthermore, both blade elements were prescribed zero lift to prevent their respective wake from affecting the simulation. A starting (span) vortex with a prescribed strength $\left(\Gamma_{S}=+\Gamma\right)$ was then released from the upwind blade element and allowed to freely convect in the flow field. As expected, the downwind blade element experienced an initial downwash followed by an upwash as shown in Figure 4.4(a). In addition, to verify that the vortex direction is also accounted for, an approaching vortex with "negative" strength $\left(\Gamma_{S}=-\Gamma\right)$ was analyzed, which resulted in the opposite trend. 
(a) Starting Vortex

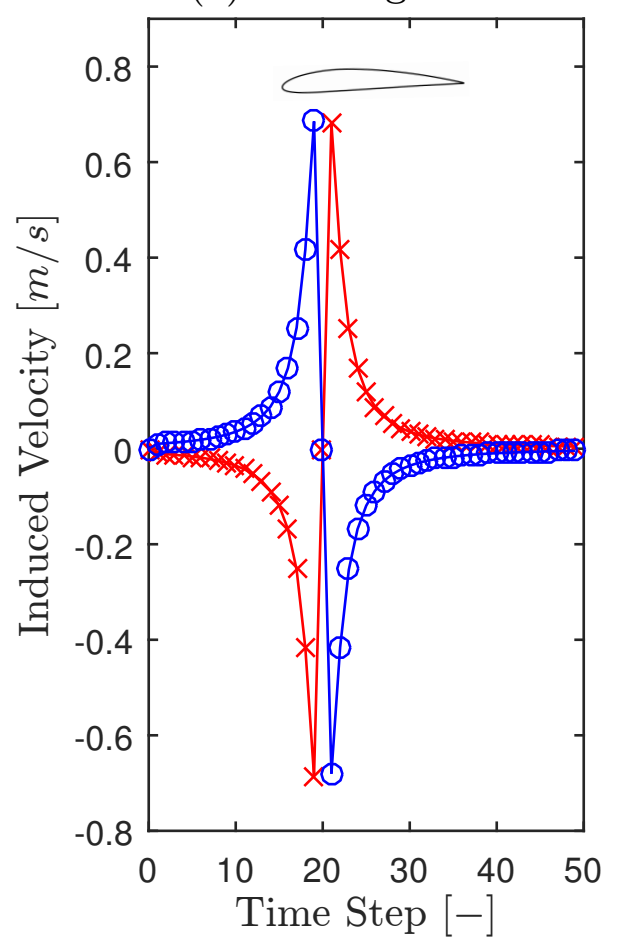

(b) Tip Vortex

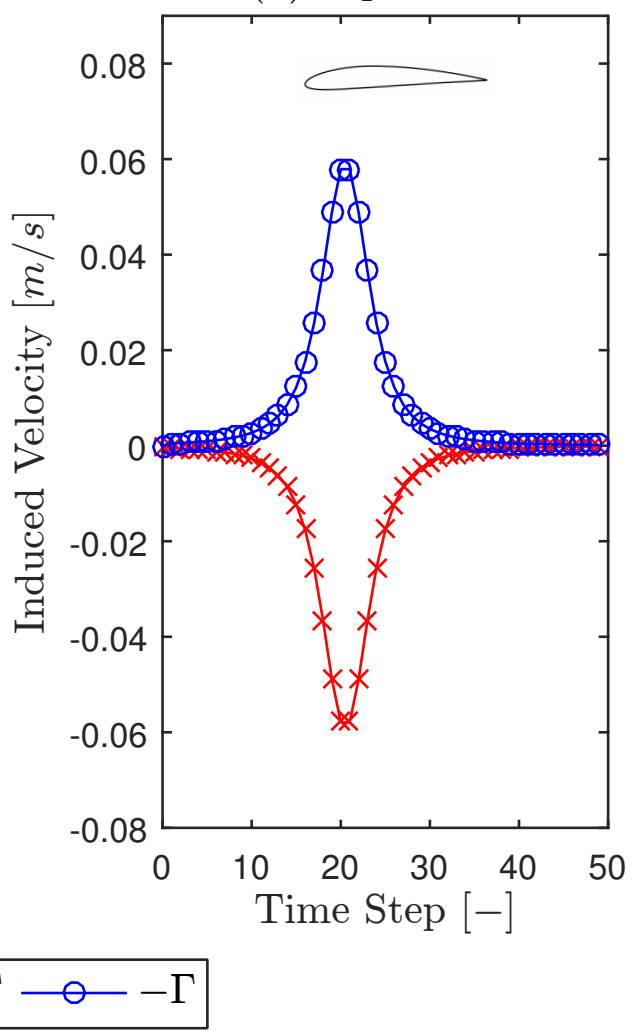

Figure 4.4: Induced velocity variation of a stationary blade element as a vortex with prescribed strength approaches. The approximate location of the stationary blade element is also shown

Using the same technique, a tip (trailing) vortex with a prescribed strength $\left(\Gamma_{S}=\Gamma\right)$ was released from the upwind blade element. As can be seen in Figure 4.4(b), a downwash velocity is induced on the blade element. As previously mentioned, the induced velocity is expected to peak when passing the leading edge of the blade element. However, since the blade elements are replaced by lifting lines (vortex filaments) in this vortex code, the peak occurs at the aerodynamic center of the element. The analysis was also performed with a "negative" strength $\left(\Gamma_{S}=-\Gamma\right)$ and as expected, the opposite trend was obtained. The interaction observed between the convecting vortex and the blade is comparable to that found in the literature [99-101]. 


\subsection{NREL Phase VI Wind Turbine}

The NREL Sequence S experiment which was briefly described in Section 2.1.1, was used as the first validation test case. As previously mentioned, NREL performed extensive controlled experiments using the two-bladed, $10 \mathrm{~m}$ diameter Phase VI turbine. The blade geometry at various spanwise sections, which can be obtained from Ref. [47], is summarized in Table 4.1. For the purpose of this work, the geometry provided by NREL will be referred to as NREL geometry. The blade is tapered and twisted with a blade tip pitch angle of $3^{\circ}$. In other words, the blade tip is pitched $3^{\circ}$ from the rotor plane. During this validation test case, various operating conditions were computed with the oncoming wind velocities ranging from $5 \mathrm{~m} / \mathrm{s}$ to $25 \mathrm{~m} / \mathrm{s}$ at a rotational velocity of $72 \mathrm{rpm}$ and $0^{\circ}$ yaw angle.

After a thorough calibration, the overall computed and measured performance of the Phase VI turbine were compared. Further comparison were performed to correlate the distribution of local angle of attack along the blade with the measured data. The following sections describe the calibration and outcome of this test case.

\subsubsection{Convergence Study}

Prior to comparing with the experimental data, a comprehensive parameter calibration was performed to determine if the solution is independent of various parameters such as the azimuthal angle increment, vortex core radius, number of discretized section, and the wake length (i.e., the total number of steps). Although a parameter calibration should be performed for all operating conditions over a wide range of samples, the calibration was only performed at a TSR of $3.75\left(V_{o}=10 \mathrm{~m} / \mathrm{s}\right)$ and 5 samples for each parameter. The latter was assumed sufficient to calibrate the NREL Phase VI model since 140 simulations would be required in place of 20 simulations, 
Table 4.1: Description of the NREL Phase VI wind turbine geometry provided in Ref. [47]

\begin{tabular}{ccccc}
\hline $\begin{array}{c}\text { Spanwise } \\
\text { Distance } \\
{[\mathbf{m}]}\end{array}$ & $\begin{array}{c}\text { Chord } \\
\text { Length } \\
{[\mathbf{m}]}\end{array}$ & $\begin{array}{c}\text { Twist }^{1} \\
{\left[{ }^{\circ}\right]}\end{array}$ & $\begin{array}{c}\text { Thickness } \\
{[\mathrm{m}]}\end{array}$ & $\begin{array}{c}\text { Airfoil } \\
\text { Section }\end{array}$ \\
\hline 0.000 & 0.218 & 0.000 & 0.218 & Cylinder \\
0.152 & 0.218 & 0.000 & 0.218 & Cylinder \\
0.375 & 0.183 & 0.000 & 0.183 & Cylinder \\
0.500 & 0.349 & 6.700 & 0.163 & Transition \\
0.559 & 0.441 & 9.900 & 0.154 & Transition \\
0.625 & 0.544 & 13.400 & 0.154 & Transition \\
0.749 & 0.737 & 20.040 & 0.154 & S809 \\
0.835 & 0.728 & 18.074 & 0.153 & S809 \\
1.002 & 0.711 & 14.292 & 0.149 & S809 \\
1.140 & 0.697 & 11.909 & 0.146 & S809 \\
1.444 & 0.666 & 7.979 & 0.140 & S809 \\
1.749 & 0.636 & 5.308 & 0.133 & S809 \\
1.835 & 0.627 & 4.712 & 0.131 & S809 \\
2.054 & 0.605 & 3.425 & 0.127 & S809 \\
2.359 & 0.574 & 2.083 & 0.120 & S809 \\
2.664 & 0.543 & 1.150 & 0.114 & S809 \\
2.677 & 0.542 & 1.115 & 0.114 & S809 \\
2.968 & 0.512 & 0.494 & 0.107 & S809 \\
3.273 & 0.482 & -0.015 & 0.101 & S809 \\
3.515 & 0.457 & -0.381 & 0.096 & S809 \\
3.578 & 0.451 & -0.475 & 0.094 & S809 \\
3.883 & 0.420 & -0.920 & 0.088 & S809 \\
4.188 & 0.389 & -1.352 & 0.081 & S809 \\
4.272 & 0.381 & -1.469 & 0.080 & S809 \\
4.492 & 0.358 & -1.775 & 0.075 & S809 \\
4.521 & 0.355 & -1.814 & 0.074 & S809 \\
\hline & & & & \\
\hline
\end{tabular}

1. Twist convention is positive towards feather 
if a calibration was performed for each operating conditions.

The effect of each parameter was analyzed by varying one parameter only in each case as can be seen in Figure 4.5. It should be noted that the baseline simulation was performed using the NREL geometry with $10^{\circ}$ azimuthal angle and $10 \%$ core radius for a wake length of $4 \mathrm{D}$ (where $\mathrm{D}$ is the rotor diameter).

\section{Azimuthal Angle Increment}

In this vortex code, time increment can be in the form of seconds or degrees. The latter was preferred to maintain the number of steps per revolution between simulations. To accurately capture the flow around the wind turbine rotor, small time increment is required, however, accuracy comes with high computational cost and therefore, a sensitivity study was performed to determine an adequate time increment for the NREL Phase VI computation. Figure 4.5(a) shows the variation in power coefficient as the time increment in degrees increases. Based on the results, an angle increment of $10^{\circ}$ was found to provide short computational runtime without affecting the accuracy of the prediction with less than $1 \%$ difference between the different cases.

\section{Vortex Core Radius}

To calibrate the vortex core radii, a sensitivity analysis with the core radius ranging from $1 \%$ to $20 \%$ was studied as shown in Figure $4.5(\mathrm{~b})$. Although the result seems to be converging at about $15-20 \%$, larger core radii tend to reduce the effect of neighboring vortices thereby negating the effect of having small timesteps as well as large number of discretized elements. At the other end of the range investigated, inconclusive results were obtained for a vortex core of $1 \%$ due to large fluctuations in power coefficient at every timestep. Therefore, core radii between $5 \%$ and $10 \%$ 
(a)

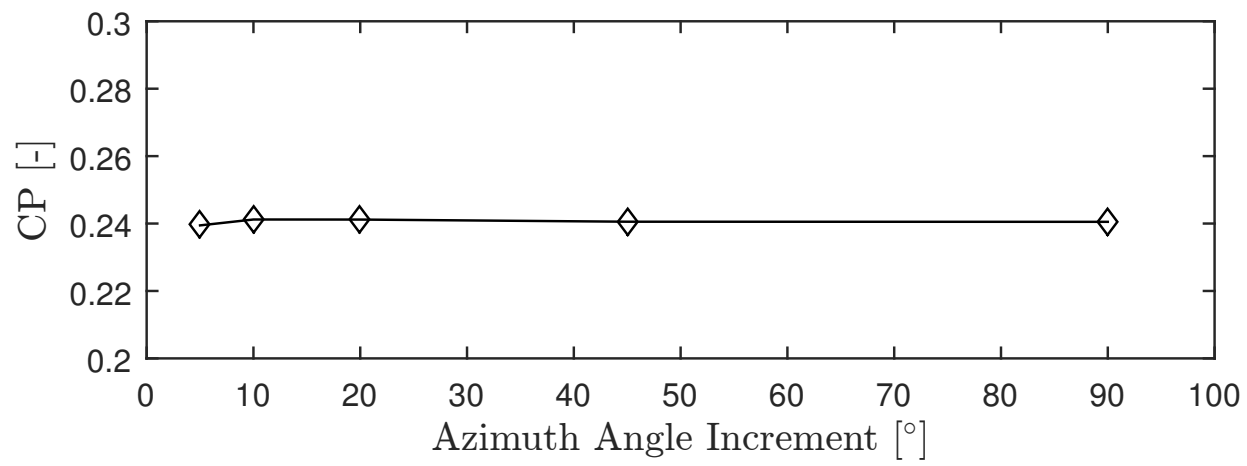

(b)

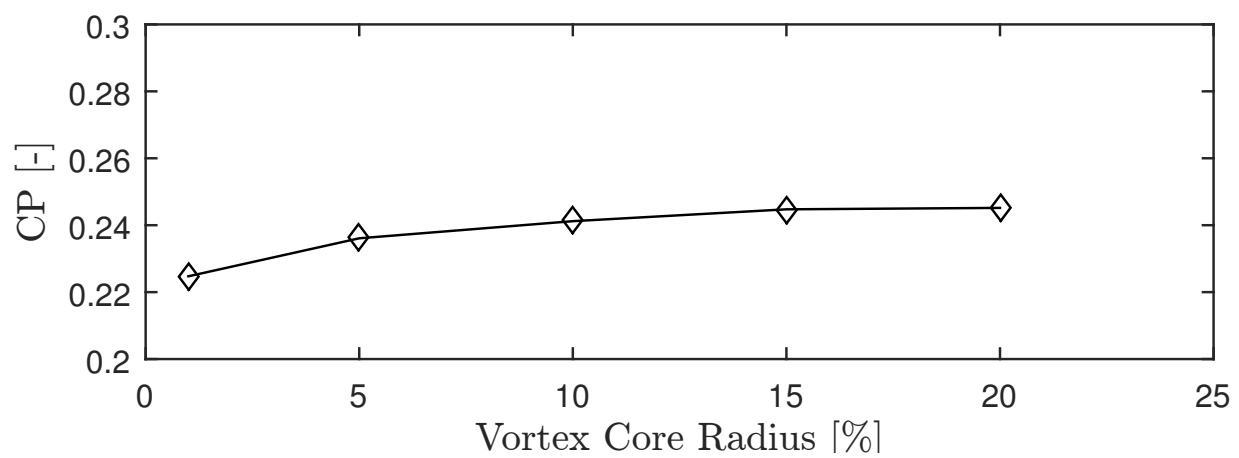

(c)

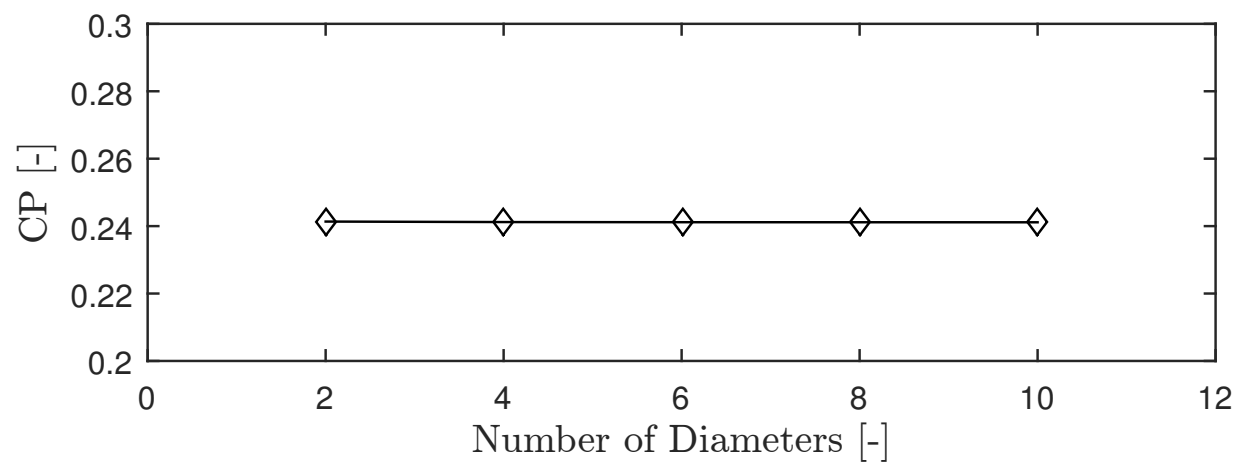

(d)

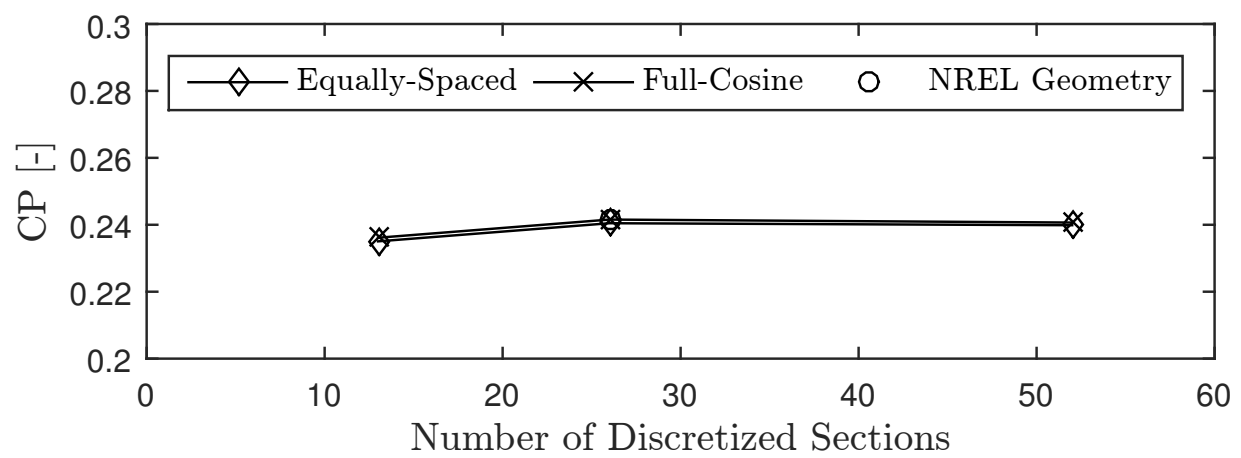

Figure 4.5: Coefficient of power as a function of the variation in (a) azimuth angle increment, (b) vortex core radius, (c) wake length, and (d) number of discretized sections 
were assumed to be adequate. This range also agrees with typical values obtained from the literature (see Section 3.6). Since the calibration was performed at only one operating condition (i.e, TSR $=3.75$ ), a conservative vortex core radius of $10 \%$ chord length was preferred.

\section{Number of Steps}

In this work, the number of steps required to obtain a converged solution is assumed to be dependent on the wake length (i.e., the distance travelled by the wake downstream of the turbine) which is usually measured in terms of rotor diameter. The NREL Phase VI was simulated until its wake reached 10D. As can be seen in Figure 4.5(c), the performance of the turbine decreases as the wake length increases. It was found that performance varied by $0.03 \%$ from $4 \mathrm{D}$ to $10 \mathrm{D}$ and since the wake produced by a wind turbine is generally fully-developed after reaching $4 \mathrm{D}$, a wake length of $4 \mathrm{D}$ was used for all simulation, unless otherwise stated. In addition, since computational cost increases drastically with the number of steps, the 4D wake length assumption allows for shorter computational runtime. For example, it took 14 times longer to simulate the NREL Phase VI from a wake length of 4D to 10D.

\section{Number of Discretized Sections}

To analyzed the effect of the number of discretized section and the discretization scheme used to model the wind turbine blades, three test cases with 13, 26, and 52 sections discretized using an equally-spaced or a full-cosine distribution (see Appendix A) were investigated. Since the inboard sections typically generate little to no torque, the discretization scheme was applied along the blade region consisting of S809 airfoil sections only (see Table 4.1). As can be seen in Figure 4.5(d), both discretization schemes showed similar trend with about $0.20 \%$ difference as the number of section 
increases from 26 to 52 . In addition, with more sections at the tip region using the full-cosine distribution, a $0.35 \%-0.45 \%$ higher performance is predicted.

The NREL geometry was also used to compute the performance of the Phase VI turbine. This resulted in a $0.15 \%$ difference with the 26 full-cosine distribution and therefore, it was assumed to provide the necessary details in the performance prediction of the NREL Phase VI turbine. Furthermore, it is believed that the use of the NREL geometry provides a better comparison with other performance codes since most codes, available in the literature, employed the NREL geometry without any discretization scheme.

\subsubsection{Comparison with Experimental Data}

The performance of the Phase VI turbine was computed using DR_HAWT based on the parameters calibrated previously. Furthermore, as described in Section 3.7, the aerodynamic polars which are obtained either experimentally or numerically (e.g., XFOIL) are two-dimensional and therefore, must be corrected for rotational effects. For this validation case, four polar databases were employed namely, the corrected (using Snel et al. and Du \& Selig 3D corrections) and uncorrected (2D) experimental data obtained from Ref. [47], as well as the uncorrected XFOIL data. Figure 4.6 shows the measured and computed torque and axial thrust of the NREL Phase VI wind turbine as a function of wind velocity. The NREL Phase VI measurements were provided by Dr. Scott Schreck from NREL [52]. The measured torques were obtained from pressure and strain gauge measurements whereas the axial trust measurements were obtained from the pressure data only.

As can be seen in the torque comparison, the computed torque for all four polars showed good agreements in the pre-stall region $(5 \mathrm{~m} / \mathrm{s}$ and $7 \mathrm{~m} / \mathrm{s})$ with a maximum 

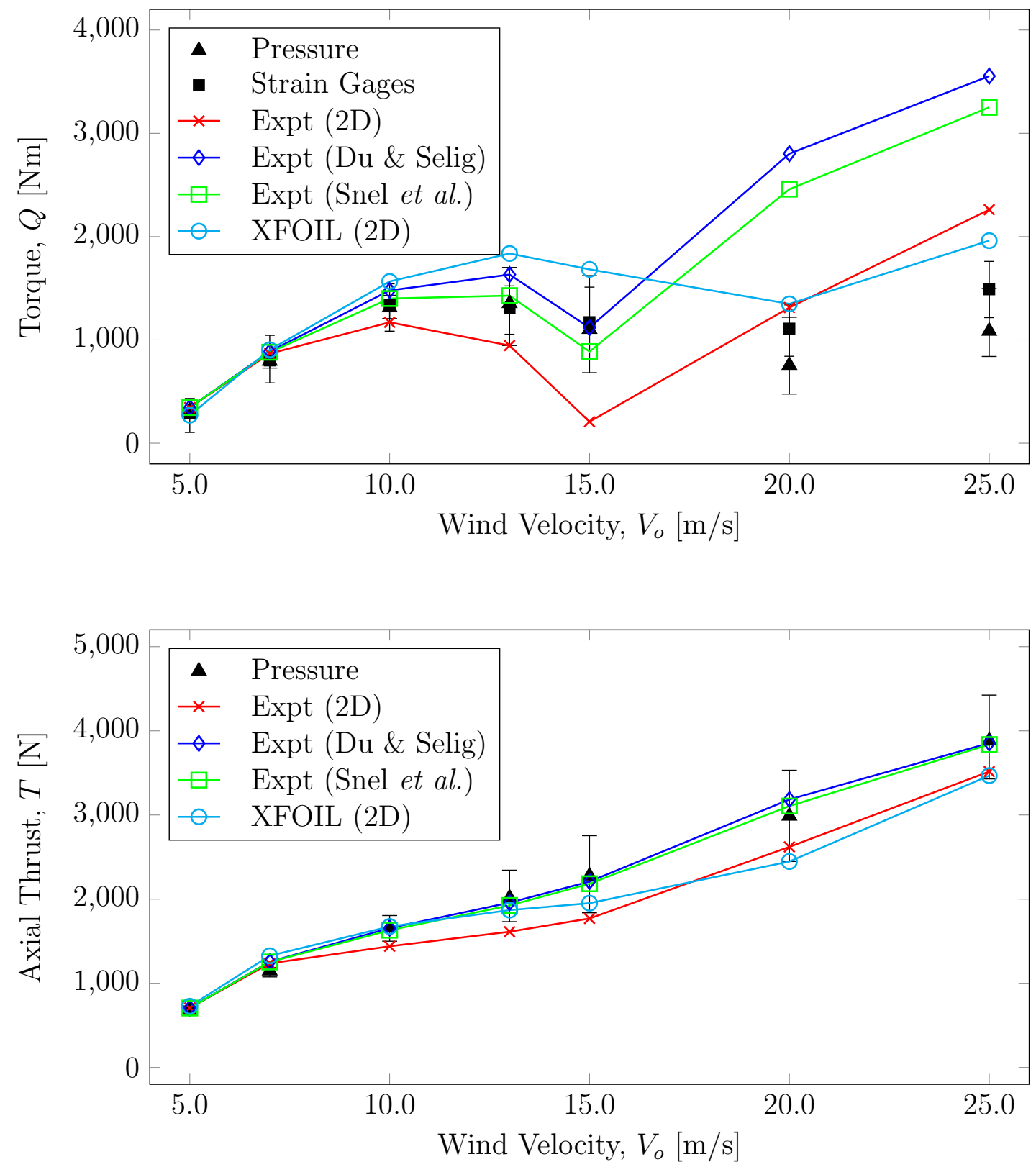

Figure 4.6: Performance prediction comparison with NREL experimental data: (a) Torque (Top) and (b) Thrust (Bottom) 
deviation of $13 \%$ with the measurements. The reason for this consistent results is because in this region, the flow over the blade is attached and therefore, the aerodynamic polars are more accurate between $2 \mathrm{D}$ wind tunnel experiments and numerical methods. In addition, there is no rotational effects acting along the span of the blade. However, in the stall region (between $10 \mathrm{~m} / \mathrm{s}$ and $15 \mathrm{~m} / \mathrm{s}$ ) where flow separation and rotational effects are present, greater deviations ranging from $5 \%$ to $52 \%$ were obtained between DR_HAWT and the NREL measurements. At high wind speeds (20 $\mathrm{m} / \mathrm{s}$ and $25 \mathrm{~m} / \mathrm{s}$ ) where most of the blade operate in deep-stall, the accuracy of the prediction is very low, with deviations ranging from $74 \%$ to $271 \%$. The reason for this large inaccuracy is that the $2 \mathrm{D}$ modeling and wind tunnel measurement of lift and drag coefficients in these flow regimes are difficult to obtain [23].

Similarly, comparing the measured and computed axial thrust, consistent results were obtained in the pre-stall region with a maximum of $15 \%$ deviation. As expected, in the stall and deep-stall region $(10 \mathrm{~m} / \mathrm{s}$ to $25 \mathrm{~m} / \mathrm{s})$, better agreements were obtained when using the corrected polars for rotational effects. Overall, the computed torque and thrust using corrected experimental polars (Du \& Selig and Snel et al.) showed a maximum of $20 \%$ (excluding deep-stall region) and $9 \%$ deviations with the NREL Phase VI measurements, respectively.

Figure 4.7 compares the computed and measured average angle of attack (AOA) distribution along the blade for several wind velocities $(5 \mathrm{~m} / \mathrm{s}$ to $15 \mathrm{~m} / \mathrm{s})$. During the NREL Phase VI experiment, the local flow angles (LFA) at several span locations along the blade $(0.34 \mathrm{R}, 0.51 \mathrm{R}, 0.67 \mathrm{R}, 0.84 \mathrm{R}$ and $0.91 \mathrm{R})$ were measured using five-hole pressure probes installed ahead of the leading edge of the blade. Moreover, an inverse BEM technique (see Appendix B) was employed to determine the AOA based on the $C_{t}$ and $C_{n}$ values from surface pressure measurements along the blade $(0.30 \mathrm{R}, 0.47 \mathrm{R}$, 
(a) $V_{o}=5 \mathrm{~m} / \mathrm{s}$

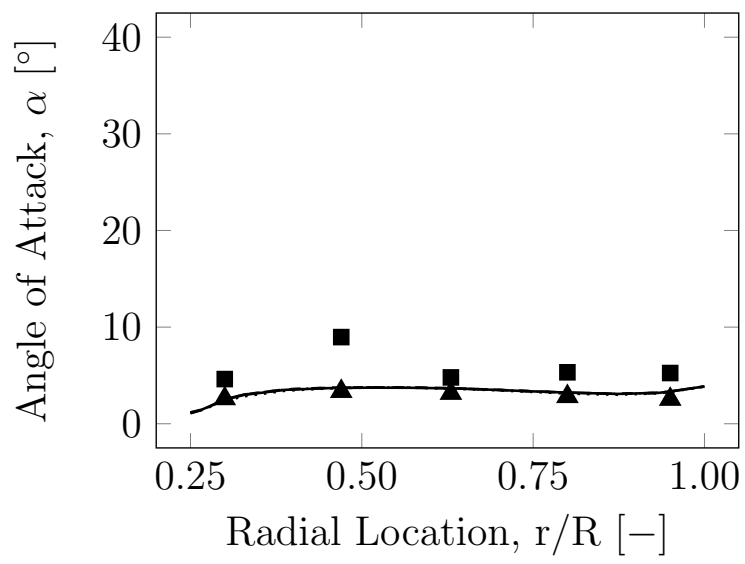

(c) $V_{o}=10 \mathrm{~m} / \mathrm{s}$

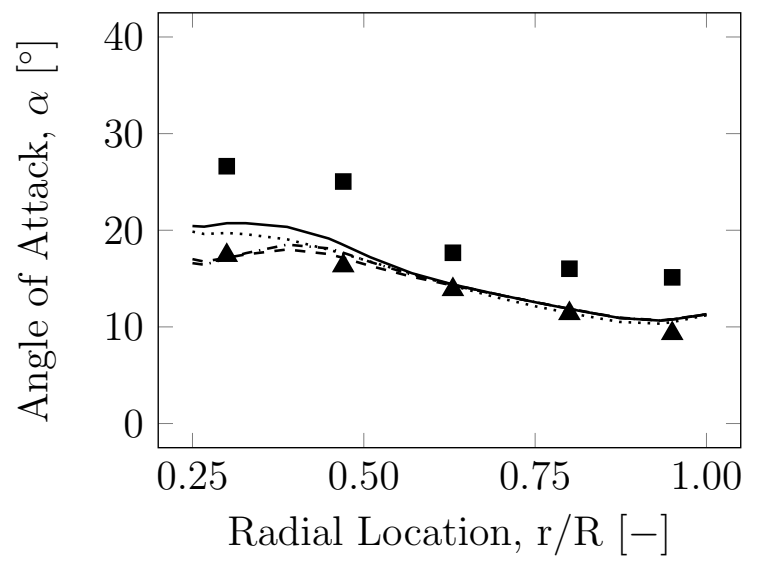

(e) $V_{o}=15 \mathrm{~m} / \mathrm{s}$

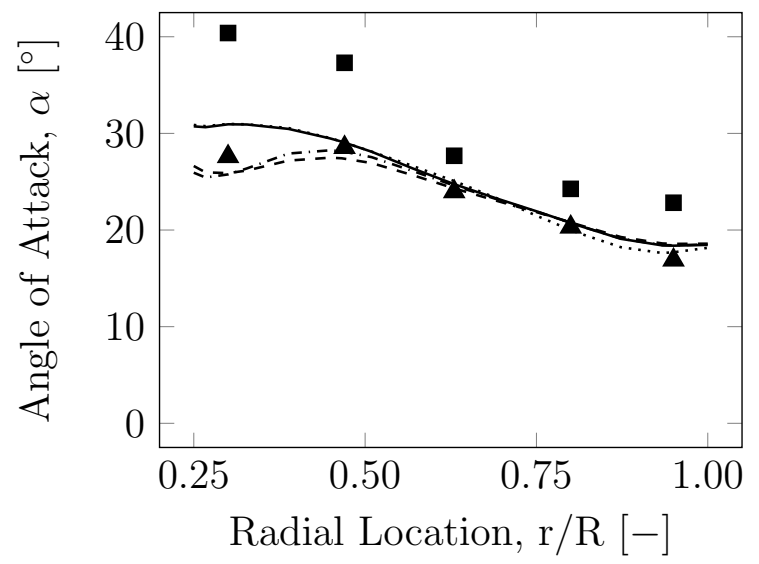

(b) $V_{o}=7 \mathrm{~m} / \mathrm{s}$

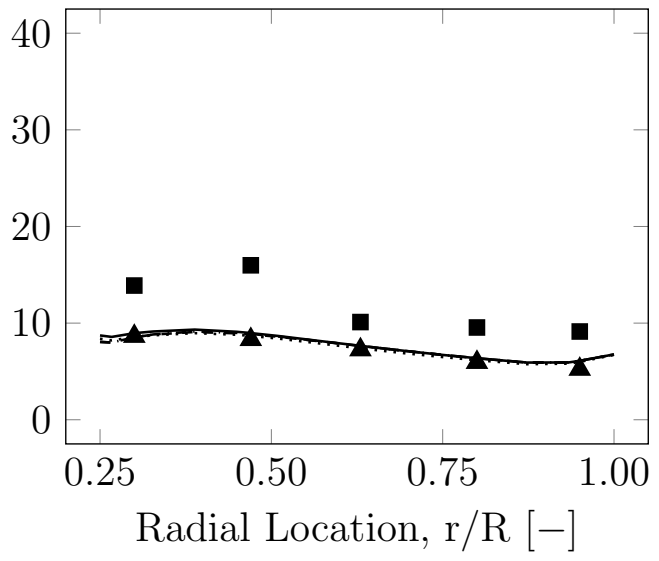

(d) $V_{o}=13 \mathrm{~m} / \mathrm{s}$

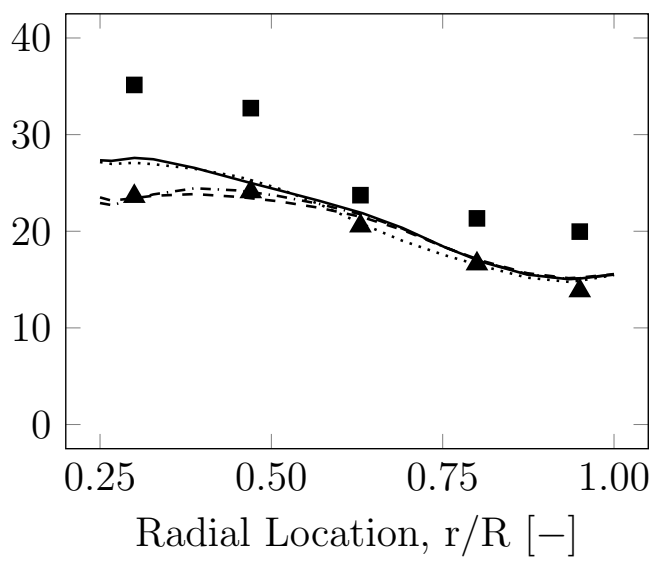

Legend:

- Inversed BEM

- Probe

- Expt (2D)

- - $\quad$ Expt (Du \& Selig)

-..- Expt (Snel et al.)

XFOIL (2D)

Figure 4.7: Comparison between computed and measured angle of attack distribution over the span of the NREL Phase VI blade 
0.63R, 0.80R and 0.95R). The large differences observed between LFA measurements and inverse BEM data (see Figure 4.7), correspond to the study performed by Sant et al. [102] using the free wake vortex code, developed at Delft University of Technology. These differences can be explained from the drawbacks of the LFA technique where 2D wind tunnel calibration of the probes may not always be accurate enough to correct the complex flow around wind turbine blades. Furthermore, the probes may alter the flow around the blade, thereby interfering with the pressure measurements [102,103].

For the purpose of this thesis, the computed AOA from DR_HAWT is compared to the inverse BEM since the LFA measurement technique used by NREL may have a considerable amount of error associated with it $[102,104]$. As can be seen in Figure 4.7, the computed AOA displays good agreement when compared to the inverse BEM data with the exception of the inboard region at higher oncoming wind velocities. As expected, the computed AOA with 2D polars exhibit large differences due to the effect of rotation and flow separation and when the 3D corrections are applied, the flow field in the inboard region is captured more accurately.

In summary, DR_HAWT accurately predicted the performance of the NREL Phase VI wind turbine with the exception of the deep-stall region. Additionally, it was observed that the accuracy of the prediction is highly dependent on the aerodynamic polars input in DR_HAWT.

\subsection{MEXICO Wind Turbine}

To further validate the vortex code, the three-bladed wind turbine which was performed within the framework of the EU FP5 project MEXICO, was chosen for its extensive coverage of the entire operational range, from turbulent wake state to stalled conditions. This $4.5 \mathrm{~m}$ diameter rotor has a standard tip-speed of $100 \mathrm{~m} / \mathrm{s}$, which 


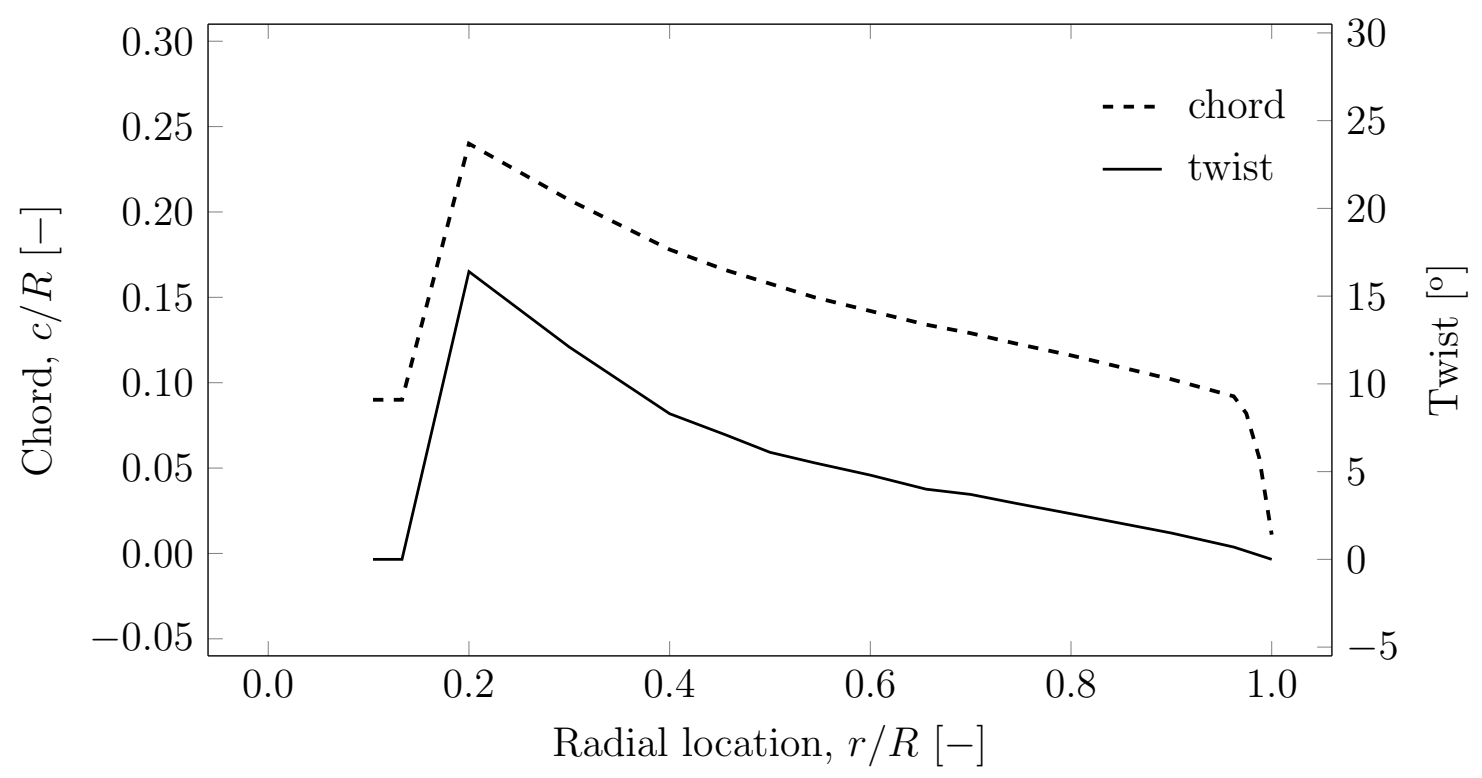

Figure 4.8: MEXICO blade chord length and twist distribution

maximizes typical tip Reynolds number while maintaining the Mach number below 0.3. The blades are twisted and tapered with chord and twist distributions as illustrated in Figure 4.8. As described in Section 2.1.2, the geometry of the blade consists of three different airfoil sections (DU91-W2-250, RISO-A1-21, and NACA 64-418). The DU91-W2-250 airfoil is applied from 20\% to $45.6 \%$ span, the RISO-A1-21 airfoil from $54.4 \%$ to $65.6 \%$ span and the NACA $64-418$ airfoil outboard of $74.4 \%$ span.

The analysis was carried out using the non-yawed configuration with a pitch angle of $-2.3^{\circ}$ at various operating conditions: wind velocity ranging from $10 \mathrm{~m} / \mathrm{s}$ to $24 \mathrm{~m} / \mathrm{s}$ and rotational speed of $424 \mathrm{rpm}$. Similar to the NREL Phase VI validation, a parameter calibration was performed prior to computing the performance of the MEXICO turbine. Based on the results (not shown here), the blades were discretized with 21 sections following the data provided in Ref. [53]. The turbine was modeled with a vortex core radius of $5 \%$ and azimuthal increment of $10^{\circ}$. In addition, the computation was assumed to have reached a converged value after traveling $4 \mathrm{D}$ downstream of the turbine. 
Figure 4.9 displays the computed and measured torque and axial thrust of the MEXICO wind turbine. The aerodynamic polar database which was based on the experimental 2D polars provided by Dr. Schepers [55], was either uncorrected (2D) or corrected using either the Snel et al. or the Du \& Selig 3D corrections. As can be seen in both figures, a large over-prediction was obtained for the pre-stall regime $(10 \mathrm{~m} / \mathrm{s}$ and $15 \mathrm{~m} / \mathrm{s})$ whereas better accuracy was obtained for the stall regime (24 $\mathrm{m} / \mathrm{s}$ ) with the corrected polars. Although a large discrepancy can be observed, the DR_HAWT's prediction falls within that of other codes from simple BEM to more sophisticated CFD (see Figures 2.5 and 2.6).

Furthermore, the local angle of attack over the span of the blade was compared with the measured data. Using the inversed BEM method described in Appendix $\mathrm{B}$, the angle of attack was computed from the tangential and normal forces at 5 spanwise locations on the blade. The comparison shown in Figure 4.10, demonstrates good agreement over the range of operating conditions investigated. Furthermore, for the stalled regime $\left(V_{o}=24 \mathrm{~m} / \mathrm{s}\right)$, the rotational effect was well captured when the $3 \mathrm{D}$ corrections were used. Despite the overestimation for the torque and axial thrust, the good agreement shown with the measured angle of attack outlines the ability of DR_HAWT to accurately predict the performance of HAWT, provided that a comprehensive calibration is carried out.

\subsection{Dual-Rotor Validation}

In the current work, several attempts in obtaining dual-rotor wind turbine (DRWT) wind tunnel measurements were pursued, however, with the limited resources and time available during this thesis, strict dual-rotor validation case was not performed. 

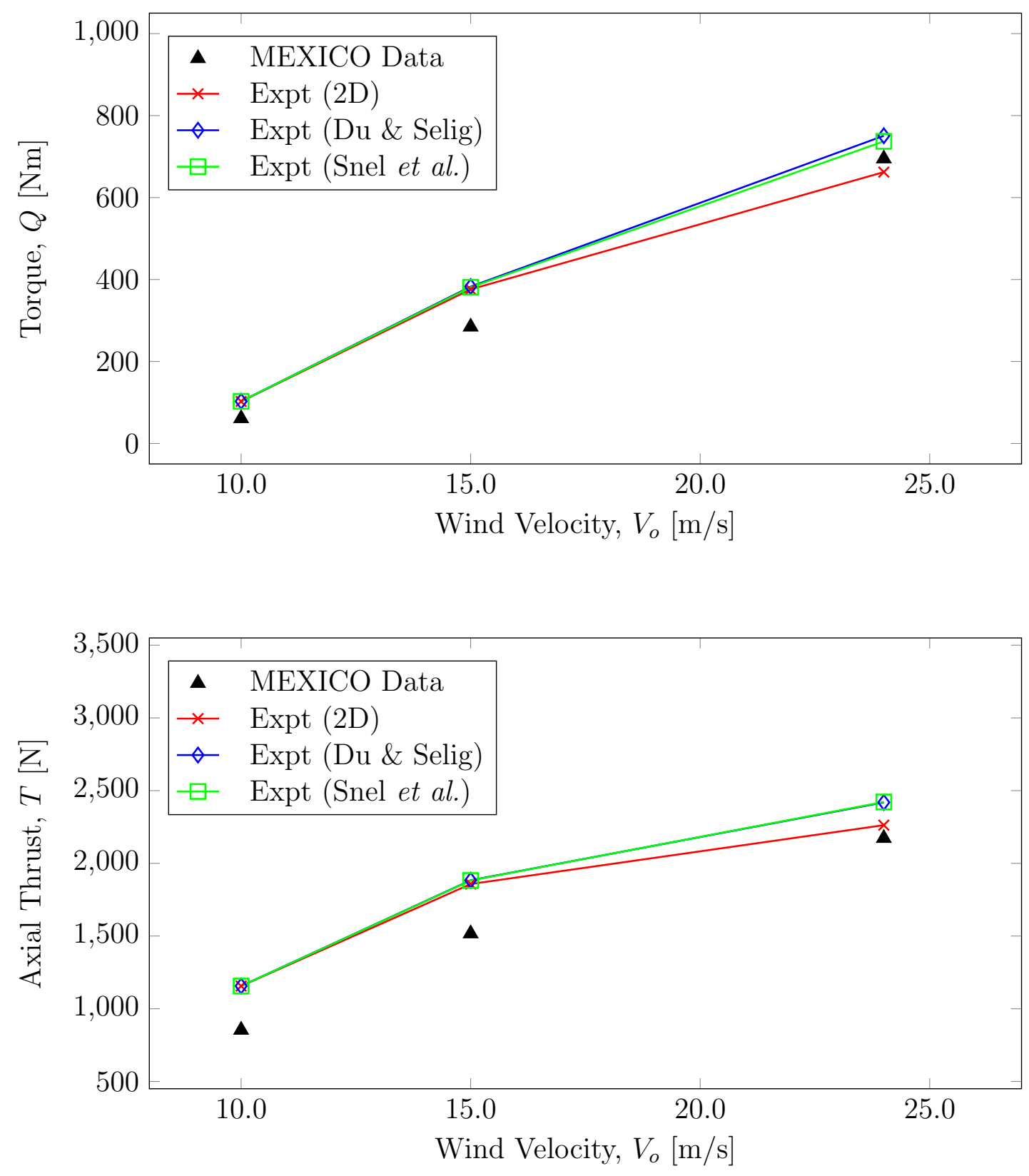

Figure 4.9: Performance prediction comparison with MEXICO experimental data: (a) Torque (Top) and (b) axial thrust (Bottom) 
(a) $V_{o}=10 \mathrm{~m} / \mathrm{s}$

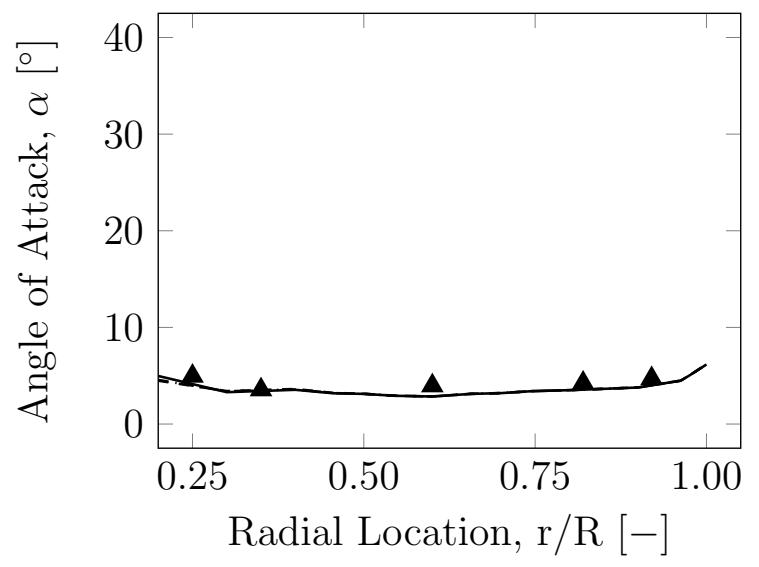

(c) $V_{o}=24 \mathrm{~m} / \mathrm{s}$

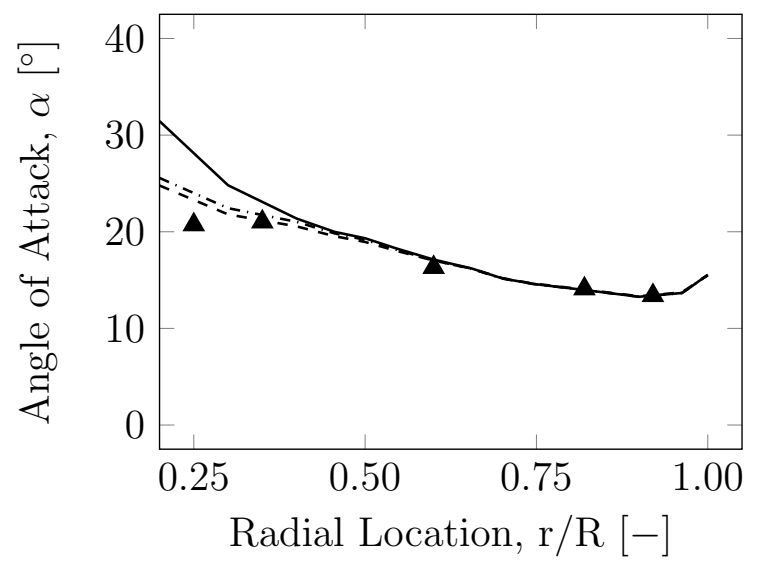

(b) $V_{o}=15 \mathrm{~m} / \mathrm{s}$

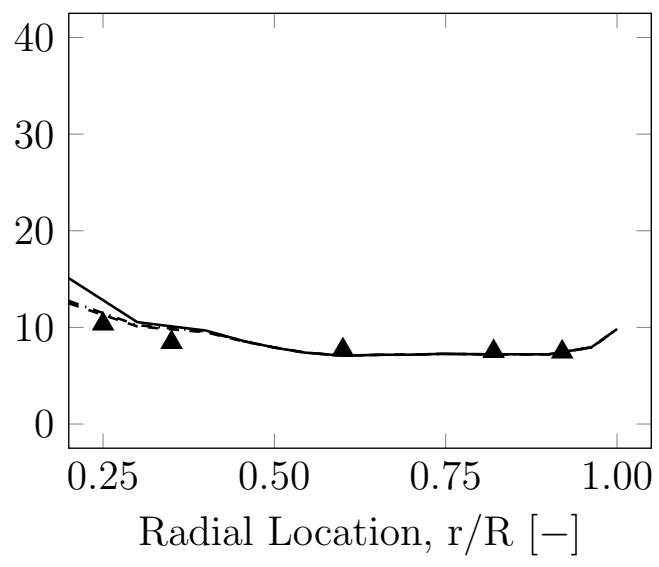

Legend:

- Inversed BEM

- Expt (2D)

- - $\quad$ Expt (Du \& Selig)

-... Expt (Snel et al.)

Figure 4.10: Comparison between computed and measured angle of attack distribution over the span of the MEXICO blade 
A wind tunnel experiment, using similar rotor design to the MAAE rotor used by McTavish during his PhD thesis (see Ref. [105]), was performed in the atmospheric boundary layer wind tunnel at Carleton University. The tapered and twisted blade consisted of Eppler 387 airfoil. Since the aerodynamic polars were not available at such low Reynolds numbers, the in-house measurements could not be used to validate DR_HAWT. Furthermore, an approach was made to initiate a collaboration with Dr. Hui Hu from Iowa State University and is yet to be finalized. With the extent of the study, it is believed that the verification and validation test cases previously described, are sufficient to provide an insight on the DRWT performance. 


\section{Chapter 5}

\section{Study Approach and Results}

This chapter describes the numerical study to identify potential dimensionless parameters for dual-rotor wind turbine (DRWT). Based on some important DRWT parameters such as the rotor speeds, rotor diameters and the distance between the rotors, three dimensionless parameters were derived using the Buckingham Pi theorem. Hypothetical DRWT models were created using geometrically-scaled National Renewable Energy Laboratory (NREL) Phase VI rotor geometry and operating conditions in order to confirm the validity of these parameters. Each DRWT was modeled and computed in DR_HAWT using aerodynamic polars from XFOIL. To compare the performance of each turbine, their output power is normalized with respect to the output power of equivalent single-rotor wind turbines (SRWTs).

\subsection{Dimensional Analysis}

The overall performance of a wind turbine can be defined as the sum of its properties which, at a given operating conditions, determine its annual energy production (AEP), lifetime, production cost and maintenance cost, etc.. However, from a purely aerodynamic point of view, the performance of a wind turbine can be defined based 
on the rotor swept area (rotor diameter) and its operating condition (oncoming wind speed and rotational speed). Similarly, as illustrated in Figure 5.1, the performance of dual-rotor wind turbines can be characterized as a function of the oncoming wind velocity $\left(V_{o}\right)$, the rotor angular velocities $\left(\omega_{1}\right.$ and $\left.\omega_{2}\right)$, the rotor diameters $\left(D_{1}\right.$ and $D_{2}$ ), and the distance separating the upwind and downwind rotor $(L)$. Other flow conditions considered are the fluid density $(\rho)$ and viscosity $(\mu)$. The dependent and independent variables considered in the dimensional analysis for dual-rotor wind turbines, are summarized in Table 5.1. Note that subscripts 1 and 2 which are associated to the rotor diameter and angular velocity, represent the upwind and downwind rotors, respectively.

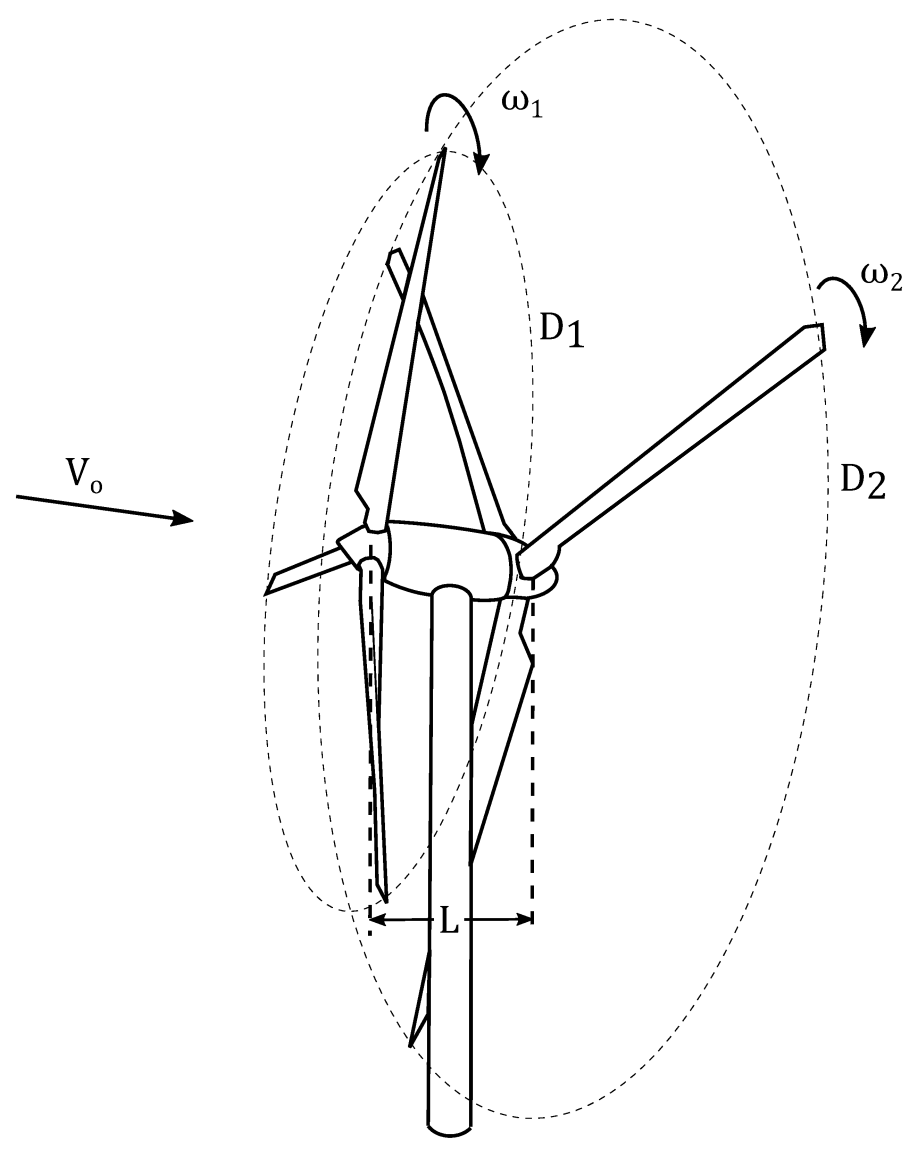

Figure 5.1: Schematic of the dual-rotor horizontal axis wind turbine's performance parameters used in the present study 
Table 5.1: Summary of dependent and independent variables for DRWT

\begin{tabular}{lll}
\hline Variable & Description & Dimensions \\
\hline$D_{1}, D_{2}$ & Rotor diameters & $L$ \\
$\omega_{1}, \omega_{2}$ & Rotor angular velocity & $T^{-1}$ \\
$L$ & Separation distance & $L$ \\
$V_{o}$ & Oncoming wind velocity & $L T^{-1}$ \\
$\rho$ & Fluid density & $M L^{-3}$ \\
$\mu$ & Fluid viscosity & $M L^{-1} T^{-1}$ \\
$P_{\text {out }}$ & Power generated & $M L^{2} T^{-3}$ \\
\hline
\end{tabular}

By using the Buckingham Pi Theorem, six dimensionless numbers were derived from the 9 variables listed in Table 5.1. The analysis was performed with reference to the downwind rotor (main rotor) since the upwind rotor is considered as an auxiliary rotor. These dimensionless numbers are given below:

$$
\Pi_{1}=\frac{\rho V_{o} D_{2}}{\mu} ; \quad \Pi_{2}=\frac{\omega_{2} D_{2}}{V_{o}} ; \quad \Pi_{3}=\frac{P_{\text {out }}}{\rho D_{2}^{2} V_{o}^{3}} ; \quad \Pi_{4}=\frac{D_{1}}{D_{2}} ; \quad \Pi_{5}=\frac{L}{D_{2}} ; \quad \Pi_{6}=\frac{\omega_{1} D_{2}}{V_{o}}
$$

where $\Pi_{1}, \Pi_{2}$, and $\Pi_{3}$ are similar to the Reynolds number $\left(R e=\left(\rho V_{o} D_{2}\right) / \mu\right)$, tipspeed ratio $\left(\lambda=\left(\omega_{2} R_{2}\right) / V_{o}\right)$, and power coefficient $\left(C_{P}=P_{\text {out }} /\left(0.5 \rho \pi R_{2}^{2} V_{o}^{3}\right)\right)$ with reference to the downwind rotor, respectively. The aforementioned numbers are commonly used to non-dimensionalize SRWT. On the other hand, $\Pi_{4}, \Pi_{5}$, and $\Pi_{6}$ constitute geometric and operational characteristics from both the upwind and downwind rotors and therefore, since the goal of this work is to identify parameters for DRWT, these three parameters will be considered in the parametric study.

For the purpose of this thesis, the first parameter which is the ratio of the upwind rotor diameter $\left(D_{1}\right)$ and the downwind rotor diameter $\left(D_{2}\right)$ is called the diameter 
ratio (DR). The gap ratio (GR) describes the ratio of the distance between the rotors and the diameter of the downwind rotor. Finally, the combined tip-speed ratio (CTSR) is defined as the ratio of the product of the upwind angular velocity and the downwind rotor radius and the oncoming wind velocity. It should be noted that the downwind rotor radius, $R_{2}$ was used as the length scale for CTSR to be consistent with the tip-speed ratio $\left(\lambda=\omega R / V_{o}\right)$ of a SRWT. For completeness, the three potential dimensionless parameters are repeated below:

$$
\begin{gathered}
D R=\frac{D_{1}}{D_{2}} \\
G R=\frac{L}{D_{2}} \\
C T S R=\frac{\omega_{1} R_{2}}{V_{o}}
\end{gathered}
$$

Furthermore, unlike other dual-rotor studies found in the literature $[5,6,15,16]$, where the power output produced by DRWTs are compared with the main rotor (usually the downwind rotor) in single-rotor configuration, the power output of a DRWT is compared with the power generated by individual SRWTs equipped with the equivalent rotors. In other words, the normalized power $\left(P_{\text {norm }}\right)$ is given by Equation 5.4 as the ratio of the power output generated by a DRWT $\left(P_{\text {dual }}\right)$ to the sum of the power output generated by the upwind and downwind rotors in singlerotor configurations $\left(P_{\text {singles }}\right)$. This method is believed to give a better value to the potential that dual-rotor systems can provide.

$$
P_{\text {norm }}=\frac{P_{\text {dual }}}{P_{\text {singles }}}
$$




\subsection{Parametric Sweep}

Based on the 3 dimensionless parameters derived previously, a parametric study which was divided into 3 test cases (D01, D02, and D03), was performed to verify their applicability to dual-rotor wind turbines. Furthermore, the scalability (D04) of these dimensionless parameters were verified where the baseline model was scaled by a factor of 0.5 and 2. Table 5.2 summarizes the specification for each case. In each test case, two parameters were varied one at a time with the third parameter kept constant. A total of 16 hypothetical wind turbine models were created ranging from $0.25-1.00$ diameter ratio and $0.25-1.00$ gap ratio. Moreover, since counter-rotating rotors were more popular in the past and proved to be more efficient as compared to co-rotating rotors, all the simulations performed during this study employed counterrotating configuration.

Table 5.2: Summary of DRWT parametric study

\begin{tabular}{ccccc}
\hline Case & Diameter Ratio & Gap Ratio & CTSR & Scale Factor \\
\hline D01 & 0.5 & $0.25-1.00$ & $6.0-10.0$ & - \\
D02 & $0.25-1.00$ & 0.5 & $6.0-10.0$ & - \\
D03 & $0.25-1.00$ & $0.25-1.00$ & 7.5 & - \\
D04 & 0.5 & 0.5 & $6.0-10.0$ & $0.5,1,2$ \\
\hline
\end{tabular}

These models were based on the geometry and operating condition of the NREL Phase VI wind turbine provided in Ref. [47] and summarized in Section 4.3. With reference to the wind turbine design of ZEC Wind Power Copr. (i.e, 0.5 diameter and gap ratios) [18], the baseline model is equipped with a $5 \mathrm{~m}$ upwind and $10 \mathrm{~m}$ downwind rotors and a separation distance of $5 \mathrm{~m}$ between the rotors. Due to aeroacoustic noise generation, land-based HAWTs are typically constraint to operate at 
tip-speeds ranging from $75 \mathrm{~m} / \mathrm{s}$ to $80 \mathrm{~m} / \mathrm{s}$ [106]. Although the tip-speed of the NREL Phase VI rotor is $37.5 \mathrm{~m} / \mathrm{s}$, it was believed adequate to geometrically scale the Phase VI rotor with the assumption that the blade tip-speed remains constant.

For all simulations, unless otherwise stated, the blade was modeled in DR_HAWT with 26 discretized sections, as provided in Table 4.1 with the appropriate scaling factor. Similar to the NREL Phase VI validation case, a $10^{\circ}$ azimuth angle increment with $10 \%$ vortex core radius was used with the assumption that the converged solution is obtained when the wake traveled $4 D_{2}$ (4 times the diameter of the downwind rotor) downstream of the downwind rotor. In addition, the aerodynamic polars which were computed in XFOIL for Reynolds numbers ranging from $1 \times 10^{4}$ to $2 \times 10^{6}$, were employed without applying any corrections. It should also be noted that the tower and nacelle are not modeled in this study. Finally, since the performance of the DRWTs are normalized with equivalent single-rotor turbines, the latter are also modeled with similar operating and flow conditions as their respective DRWT models.

\subsection{Results and Discussion}

This section details the results the four case studies previously described (i.e., Cases D01, D02, D03 and D04). Potential trends to determine an optimal design for dualrotor wind turbines are also explained.

\subsubsection{CASE D01: Effect of Gap Ratio vs CTSR}

The effect of varying the distance separating the upwind and downwind rotors is analyzed in this case study. The baseline turbine which is equipped with a $5.0 \mathrm{~m}$ and $10.0 \mathrm{~m}$ diameter upwind and downwind rotors, respectively, was utilized with various gap ratio $(0.25-1.00)$ as illustrated in Figure 5.2. The performance of these four 
DRWT models were simulated at combined TSR of 6.0, 7.5, and 10.0. As can be seen in Figure 5.3, comparable performances were obtained between the different models with almost $100 \%$ normalized power obtained at 6.0 and 7.5 CTSR. These similar performances can be explained by analyzing the wake expansion of the upwind rotor. Figure 5.4 shows the wake visualization which was obtained from DR_HAWT, of a half-scale NREL Phase VI rotor (i.e, the upwind rotor in a single-rotor configuration) operating at a TSR of $3.75\left(V_{o}=10 \mathrm{~m} / \mathrm{s}\right.$ and $\left.\omega=144 \mathrm{rpm}\right)$. Similar to the NREL Phase VI smoke visualization, there is hardly any wake expansion due to its very low thrust values. That being said, at higher CTSR, the turbine models which were expected to perform as efficiently as the other two cases, produced only about $90 \%$ normalized power.

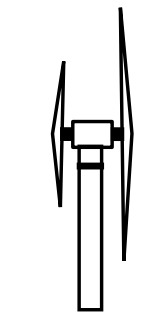

$0.25 \mathrm{GR}$

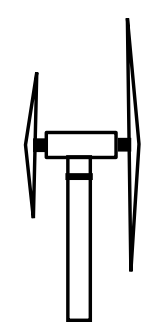

$0.50 \mathrm{GR}$

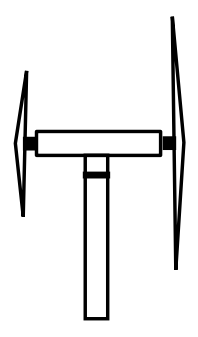

$0.75 \mathrm{GR}$

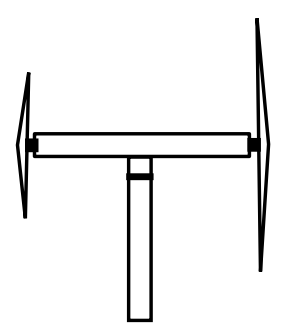

$1.00 \mathrm{GR}$

Figure 5.2: Schematic of the four DRWT models investigated in Case D01

To understand why this deficit was obtained, the power output of the upwind and downwind rotors, which are displayed in Figure 5.5, were compared to their respective equivalent single-rotor wind turbine. It can be observed that the performance of the upwind rotor for both the 6.0 and 7.5 CTSR were slightly affected at 0.25 gap ratio whereas at 10.0 CTSR, a maximum of $20 \%$ deficit in upwind power was obtained, 


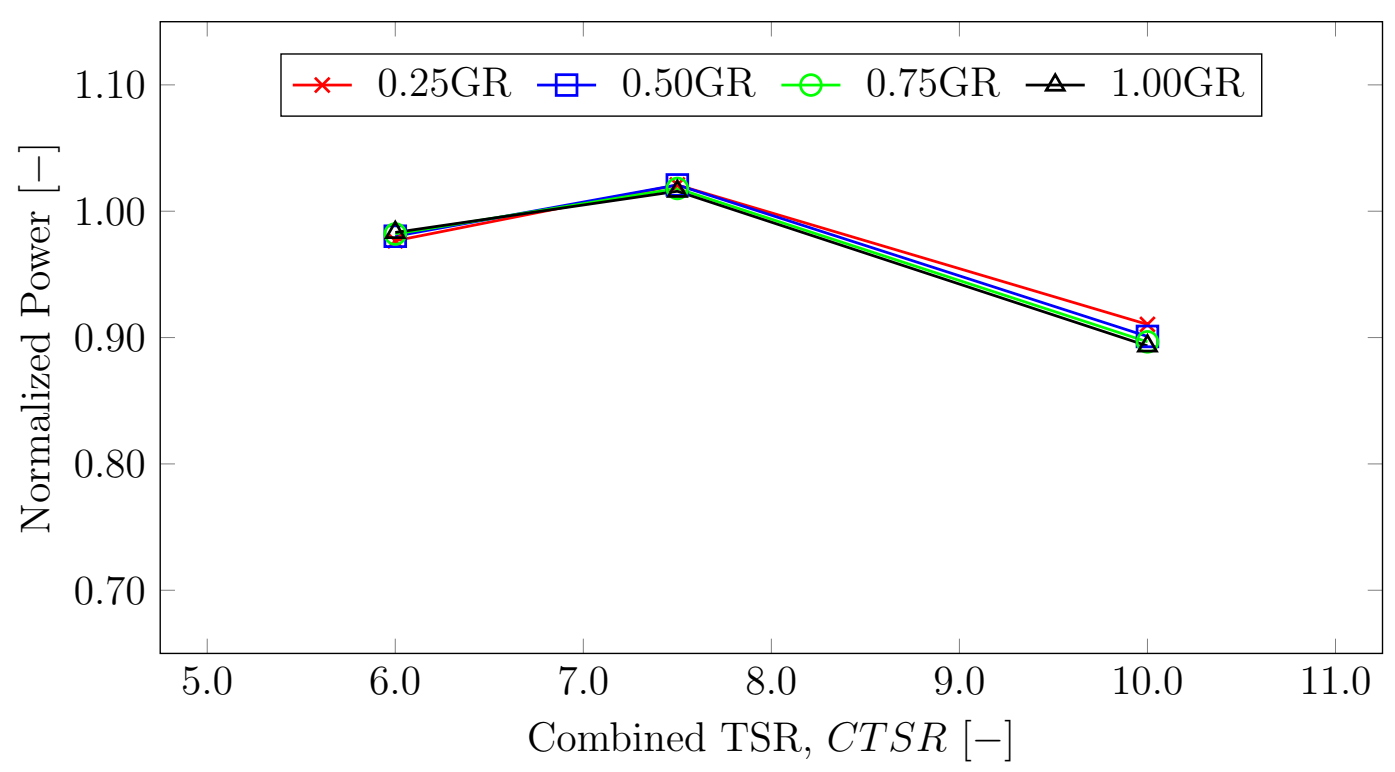

Figure 5.3: Power output comparison between four DRWT having diameter ratio of 0.50 at various gap ratios $(\mathrm{GR}=0.25,0.50,0.75$, and 1.00$)$

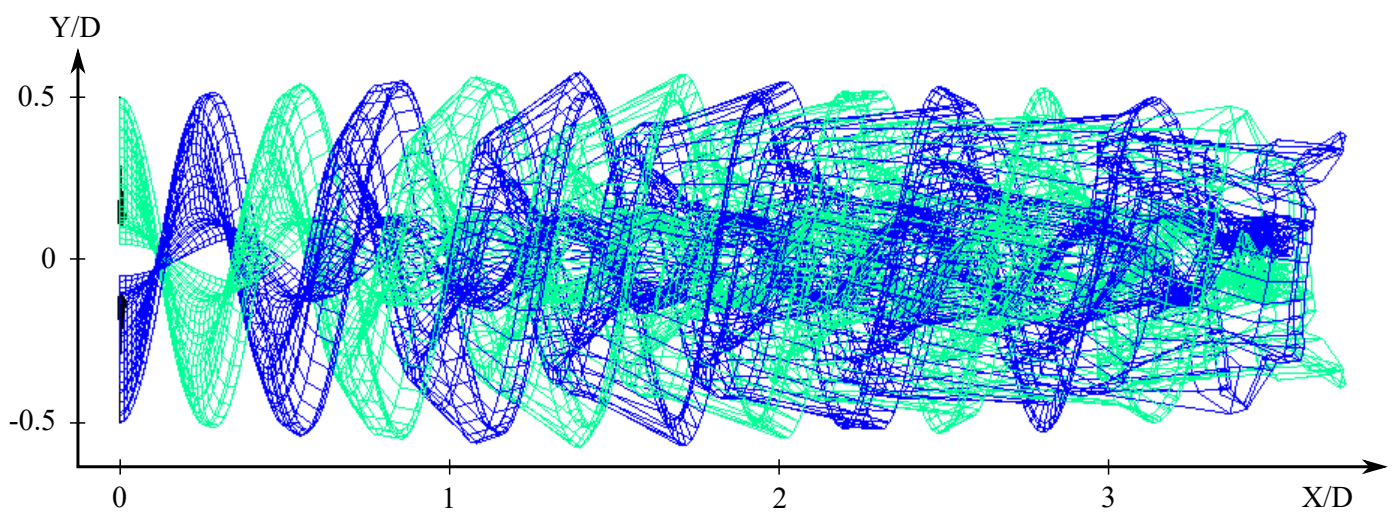

Figure 5.4: Wake visualization of a half-scaled NREL Phase VI rotor generated from DR_HAWT 


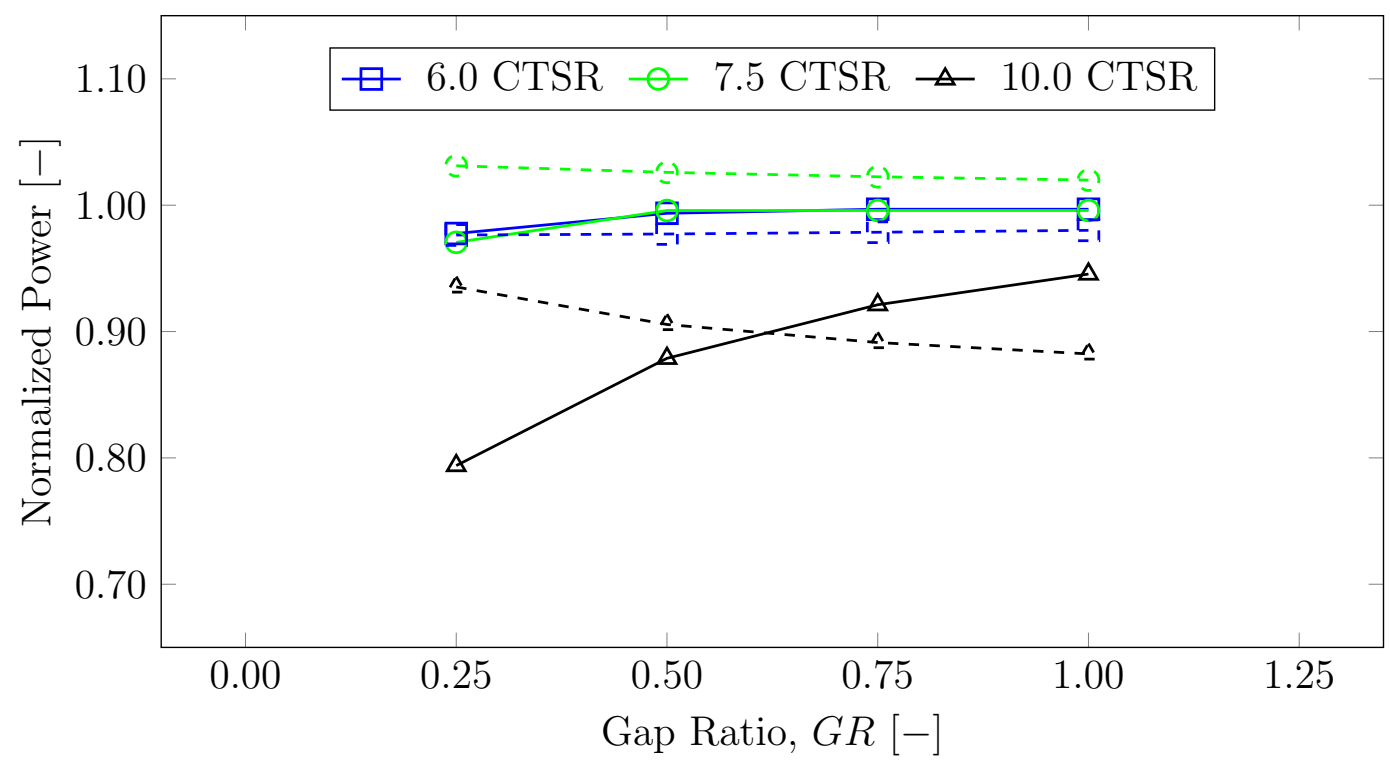

Figure 5.5: Performance of the upwind (solid lines) and downwind (dashed lines) rotors compared to their respective SRWT as a function of gap ratio

which decreases as the downwind rotor was moved further downstream. Further investigation revealed that, due to the interaction with the downwind rotor, the upwind rotor experienced similar relative velocity than its equivalent SRWT while the angle of attack (AOA) distribution for each operating condition was lower. As a result, at 10.0 CTSR, the flow around the upwind blade re-attached causing a drop in lift and therefore, lowering its performance. However, as the distance between the rotors increases, the effect becomes less pronounced thereby generating single-rotor-like performances. On the other hand, the downwind rotor performance has a reversed trend where the output power decreases with an increasing gap ratio. Similar to the upwind blade, this decreasing trend is caused by variation in AOA experienced by the downwind blade.

Moreover, as can be observed in Figure 5.5, the downwind rotor extracted more than its equivalent SRWT when operating at $7.5 \mathrm{CTSR}\left(V_{o}=10 \mathrm{~m} / \mathrm{s}\right)$. At this operating condition, the inner region of the blade in single-rotor configuration is 


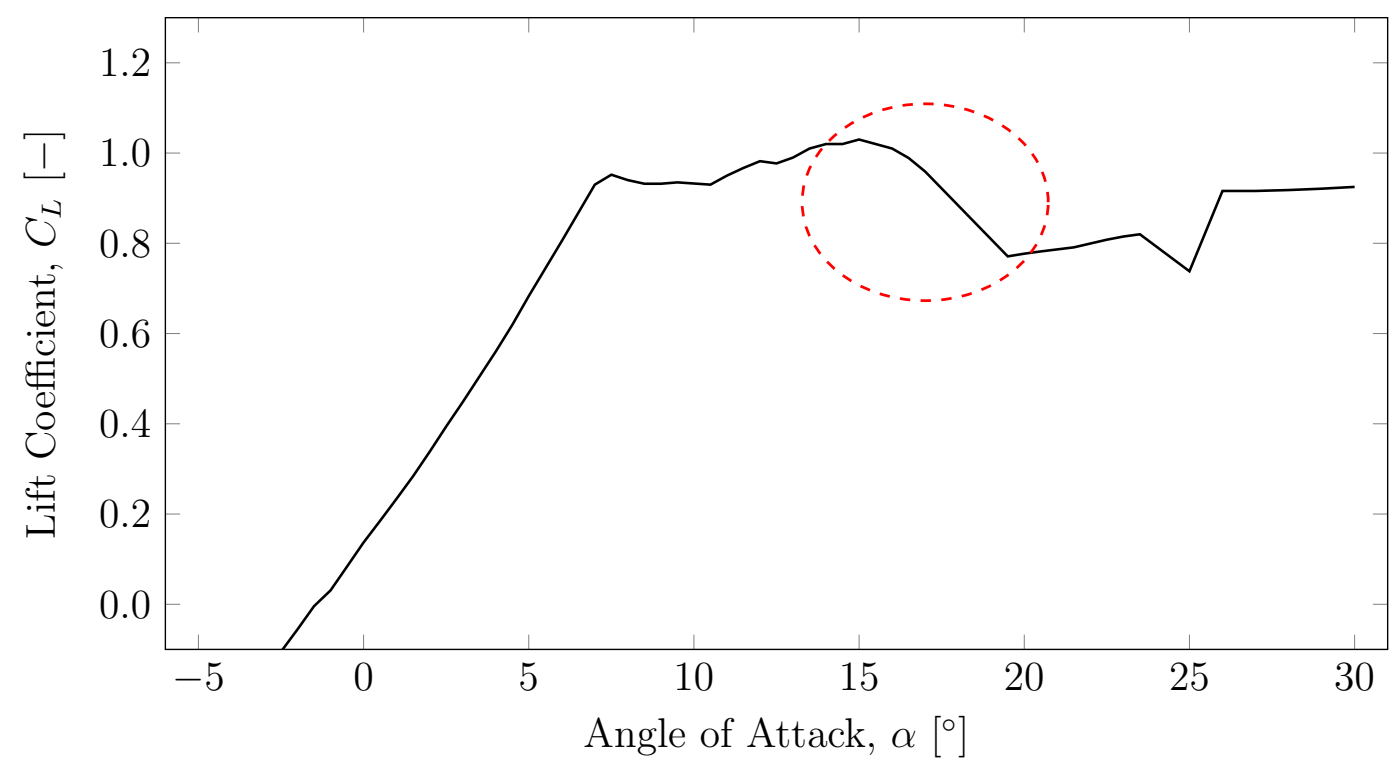

Figure 5.6: S809 lift curve at $\operatorname{Re}=360,000$

considered to be in deep-stall. However, in a dual-rotor configuration, since the angle of attack of the downwind rotor is lowered as a result of the upwind wake, it is hypothesized that at $10 \mathrm{~m} / \mathrm{s}$, the operating range of the downwind blade's inboard sections was shifted from deep-stalled to stall condition. For instance, at $40 \%$ along the span of the blade, the AOA dropped from about $18^{\circ}$ to $14^{\circ}$. Figure 5.6 shows the lift curve for the S809 airfoil at a Reynolds number of 360,000. As can be seen, this drop in AOA (circled in red) increases the lift coefficient by about $20 \%$ thereby increasing the power generation.

\subsubsection{CASE D02: Effect of Diameter Ratio vs CTSR}

The second case study investigates the effect of varying the swept area of the upwind rotor with respect to the downwind rotor. The four turbine models studied in this case, are equipped with $2.5-10.0 \mathrm{~m}$ diameter upwind rotor and a $10.0 \mathrm{~m}$ diameter downwind rotor separated by a $5.0 \mathrm{~m}$ long nacelle. A schematic of each turbine 
is shown in Figure 5.7. The overall trend, as seen in Figure 5.8, shows that more power can be extracted at lower diameter ratio with the smallest ratio generating 100\% normalized power at all CTSRs. This trend can be explained by comparing the power generated by the downwind rotor of each turbine. Figure 5.9 shows the performance of the upwind and downwind rotors as compared to their respective equivalent SRWT. Since the outer region of a rotor is typically designed to extract the most energy, the performance of the downwind rotor would be affected if the swept area of the upwind rotor overshadows the outer region of the former. Further investigation showed that the downwind rotor of the 1.0 diameter ratio appeared not to be producing any power at 10.0 CTSR (see Figure 5.9). The reason for this lack of power generation is due to a combination of the momentum loss from the upwind rotor and the fact that the freestream velocity was below the turbine's cut-in speed.

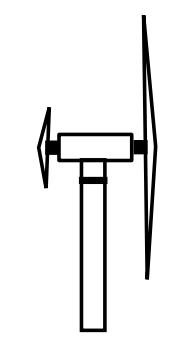

$0.25 \mathrm{DR}$

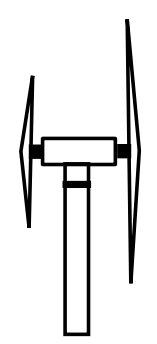

$0.50 \mathrm{DR}$

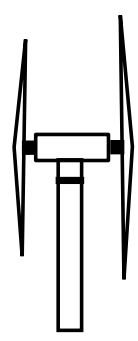

$0.75 \mathrm{DR}$

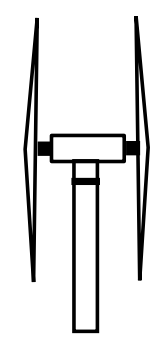

$1.00 \mathrm{DR}$

Figure 5.7: Schematic of the four DRWT models investigated in Case D02

The general effect of diameter ratio on the turbine's performance seems to be well represented in Figure 5.8, however, using combined TSR to compare the significance of this dimensionless parameter is believed to be an inadequate method. Contrary to the previous case (D01), in order to investigate similar CTSRs, large variation in the oncoming wind velocity was utilized between the turbine models since CTSR 


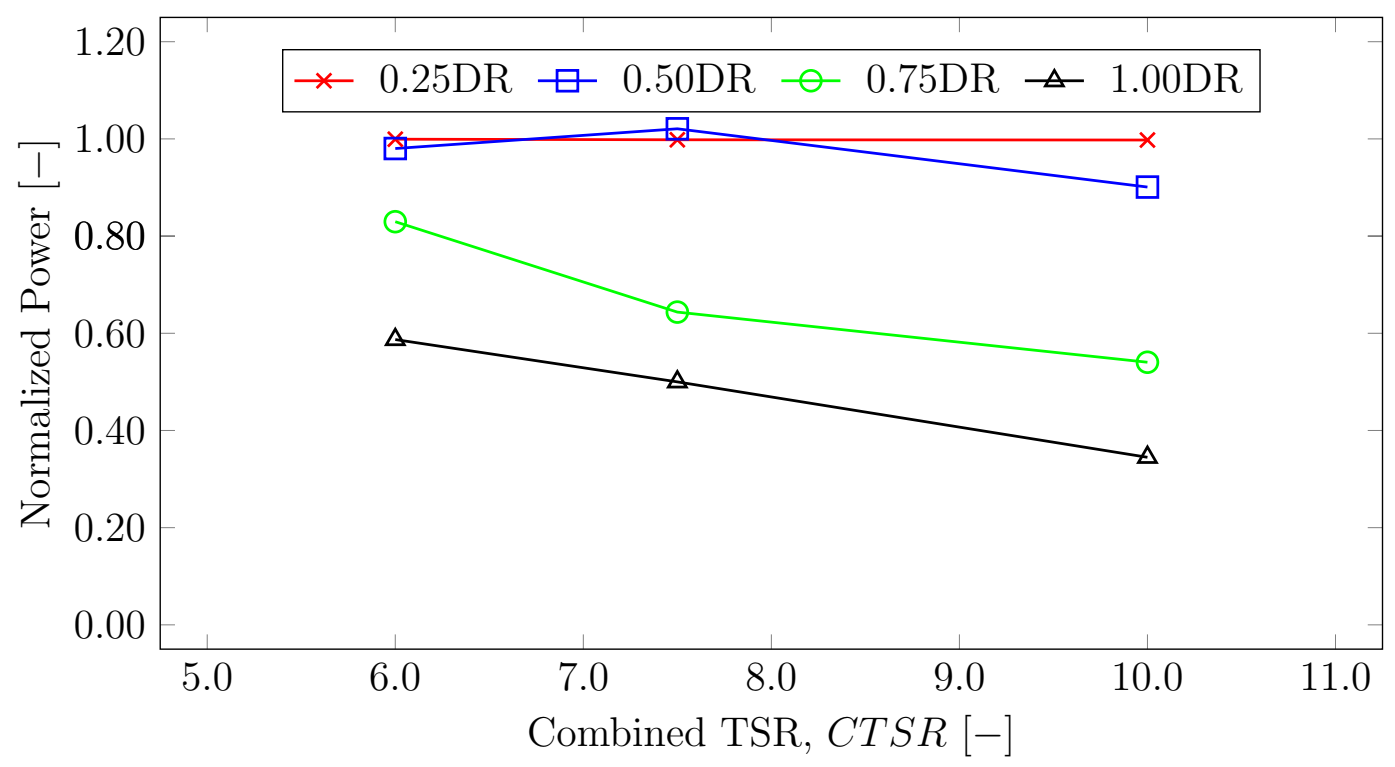

Figure 5.8: Power output comparison between four DRWT having various diameter ratios $(\mathrm{DR}=0.25,0.50,0.75$, and 1.00) and a gap ratio of 0.50 as a function of combined tip-speed ratio

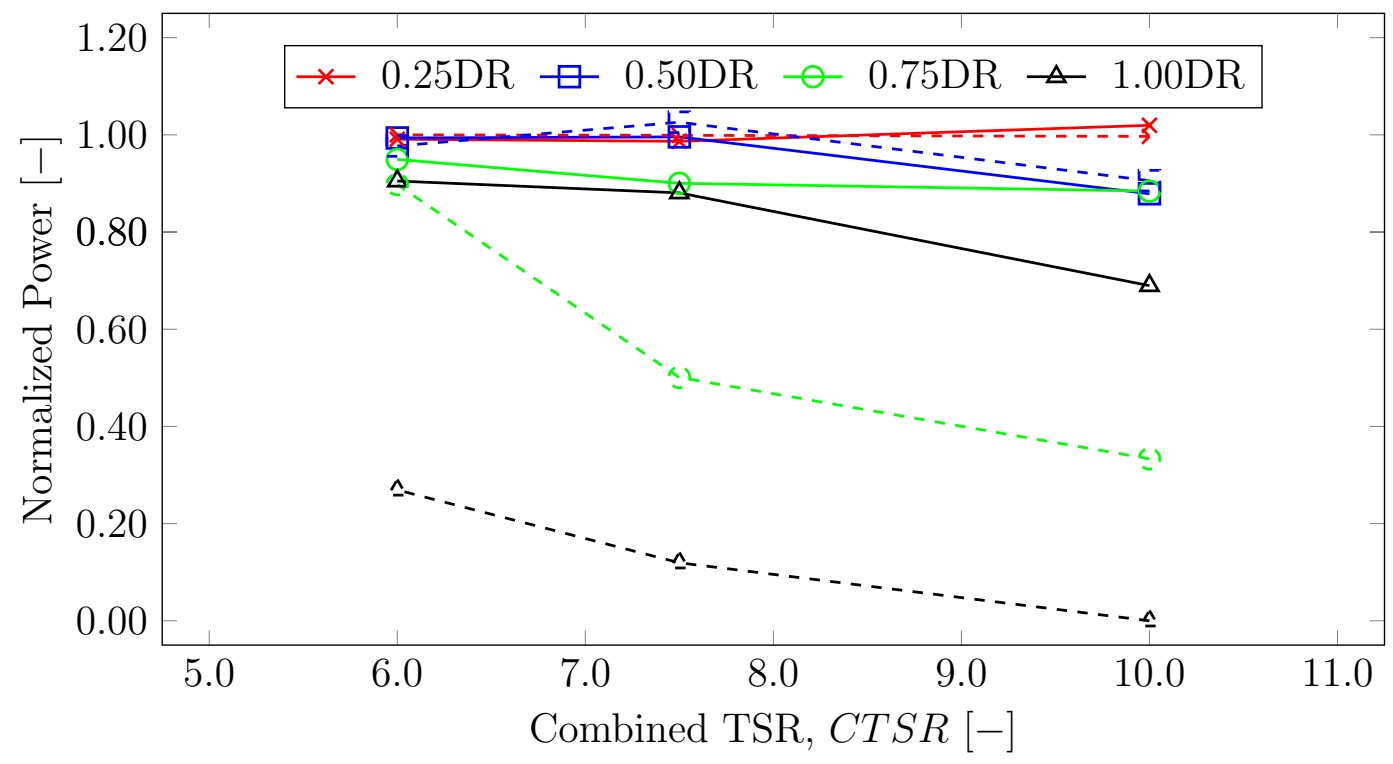

Figure 5.9: Performance of the upwind (solid lines) and downwind (dashed lines) rotors compared to their respective SRWT as a function of combined tip-speed ratio 
is a function of the upwind rotational speed, downwind rotor radius and freestream velocity (i.e., CTSR $=f\left(\omega_{1}, R_{2}, V_{o}\right)$ ). The turbines are therefore operating in various conditions from attached flows to deep-stall regions. That being said, comparing against constant wind velocity is considered to provide better representation since four turbines with different diameter ratio experiencing the same wind velocity provide a better contrast than turbines experiencing constant CTSR (i.e., different wind velocities).

To confirm the trend displayed in Figure 5.8, similar simulations were performed with oncoming wind velocities of $7.5 \mathrm{~m} / \mathrm{s}, 10.0 \mathrm{~m} / \mathrm{s}$, and $12.5 \mathrm{~m} / \mathrm{s}$. Figure 5.10 displays the normalized power of four turbine models as a function of the freestream velocity. As previously explained, the overall performance of the turbine can be observed to decrease with increasing diameter ratio at all wind speeds. As expected, the effect of the upwind wake becomes more pronounce with increasing upwind rotor diameter. In addition, similar to Case D01, the upwind turbines generated the same amount of power compared to their respective SRWT, with the exception of the models operating at $7.5 \mathrm{~m} / \mathrm{s}$. The reason for this exception is again due to the variation in AOA as described in the previous case.

The effect of diameter ratio can further be explain by analyzing the angle of attack distribution of each downwind rotor. Figure 5.11 displays the angle of attack distribution along the blade span for the downwind rotor of four different DR models and for their equivalent SRWT. It can be observed that at $0.5 \mathrm{DR}$, the reduction in $\mathrm{AOA}$ is more pronounced in the inboard section between $25 \%$ to $50 \%$ span. Although not clearly shown in this figure, the effect is not as apparent near the root since this region consists mostly of cylindrical sections where vortex filaments of lower strength are shed. In addition, the wake of the upwind rotor still affects the downwind rotor 


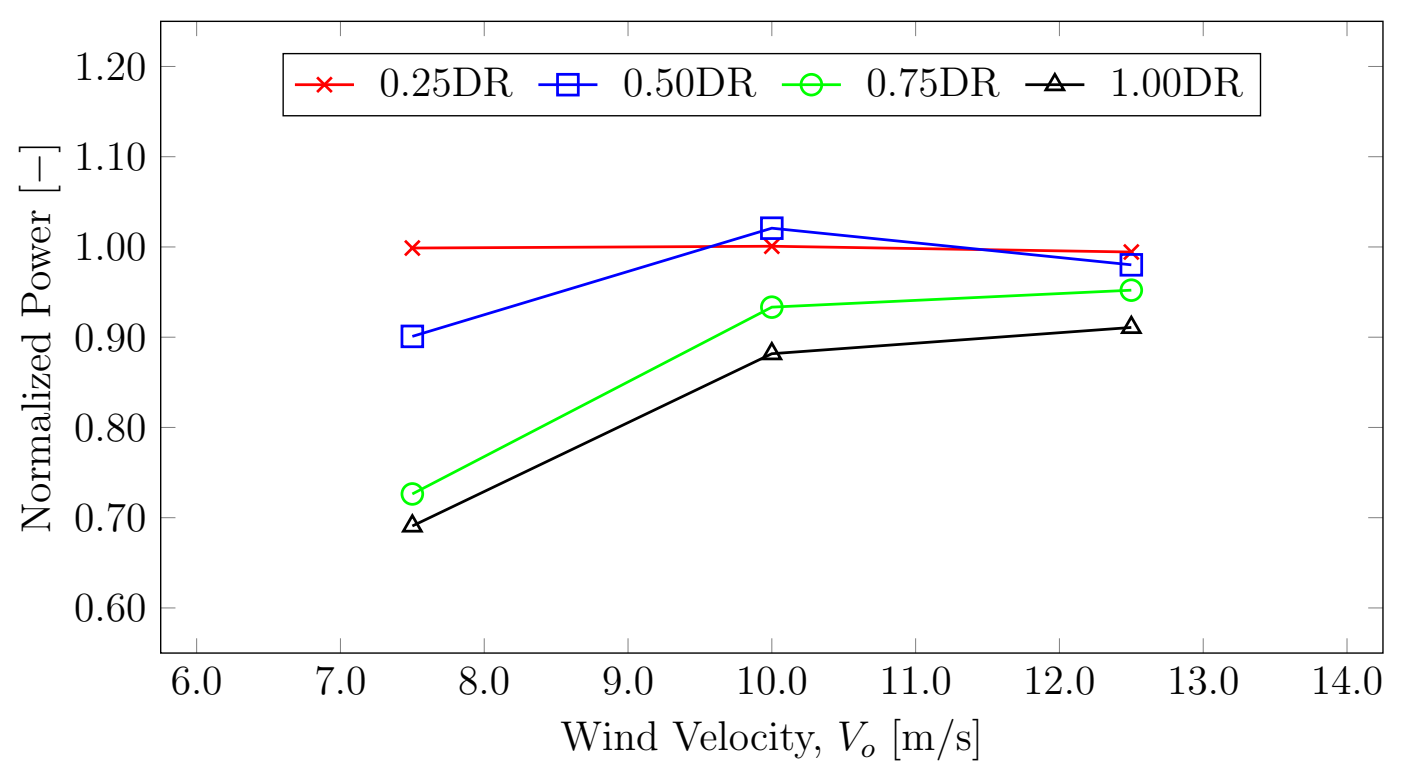

Figure 5.10: Power output comparison between four DRWT having various diameter ratios $(\mathrm{DR}=0.25,0.50,0.75$, and 1.00) and a gap ratio of 0.50 as a function of freestream velocity

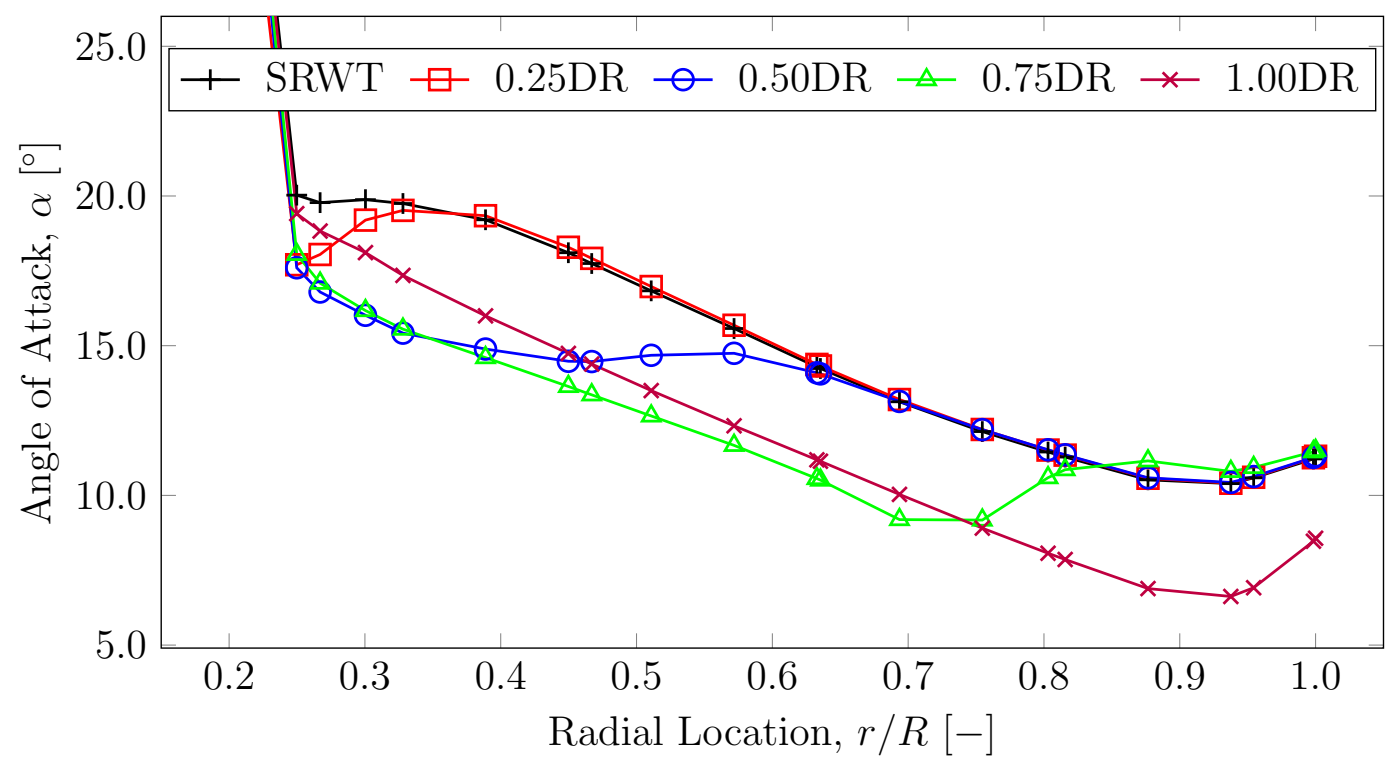

Figure 5.11: Angle of attack comparison between the downwind rotor of DRWT with diameter ratios ranging from 0.25 to 1.00 and its equivalent SRWT at a freestream velocity of $10.0 \mathrm{~m} / \mathrm{s}$ 
outer $50 \%$ span due to wake expansion which gradually dissipates nearing the blade tip. A similar trend can be observed for all models with the difference in AOA increasing as the upwind rotor grows in size.

\subsubsection{CASE D03: Effect of Diameter Ratio vs Gap Ratio}

To complement the first two cases, the effect of varying the diameter ratio as a function of the gap ratio was studied. In this case study, two set of computations were performed to verify the reasoning previously discussed, regarding using CTSR as a means of comparison. Both set of computations investigated 16 turbine models with diameter and gap ratio ranging from 0.25 to 1.00, as illustrated in Figure 5.12.

The first set of computations was performed at a constant CTSR of 7.5 to avoid simulating the downwind rotor outside its design operating range. Figure 5.13 displays the results for the turbine operating at constant CTSR. As expected, the dual-rotor systems with diameter ratios of 0.25 and 0.5 produced the best overall performance, as seen in Figure 5.13. The normalized power for each model with similar diameter ratio was also found to be fairly constant across the range of gap ratio investigated. Furthermore, similar overall trend to that obtained from the constant CTSR simulations was reported in the second set of computations (see Figure 5.14) which was performed at constant oncoming wind velocity of $10 \mathrm{~m} / \mathrm{s}$. Although different normalized powers were obtained between the two sets of computations, due to different operating conditions, this comparable trend suggests that the combined tip-speed ratio can be used to compare the general performance trend of various DRWT models.

Figure 5.15 gives the variation in power output of the upwind and downwind rotors separately as a function of gap ratio. At diameter ratio greater than 0.5 , the results showed that the power generated by the downwind rotor decreases with increasing gap 


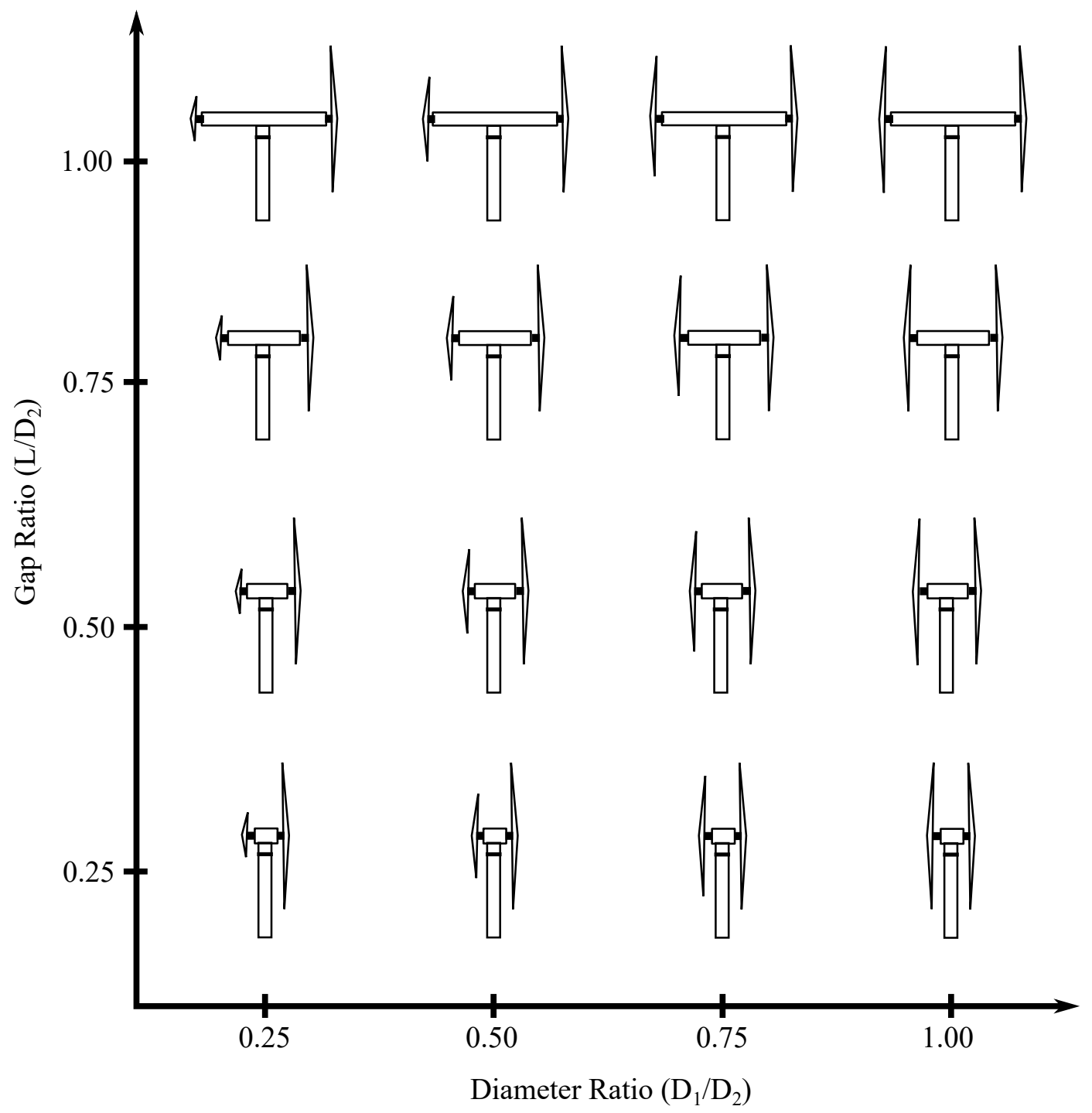

Figure 5.12: Schematic of the 16 DRWT models investigated in Case D03

ratio whereas the upwind power output increases with increasing gap ratio. Due to the interaction between the downwind rotor and the upwind wake, the power loss of the downwind rotor was found to be as high as $25 \%$ for the turbine with equivalent rotors, in comparison to the power generation of similar rotor in single-rotor configuration. Moreover, at the lowest diameter ratio investigated (i.e., $L / D_{2}=0.25$ ), the power 


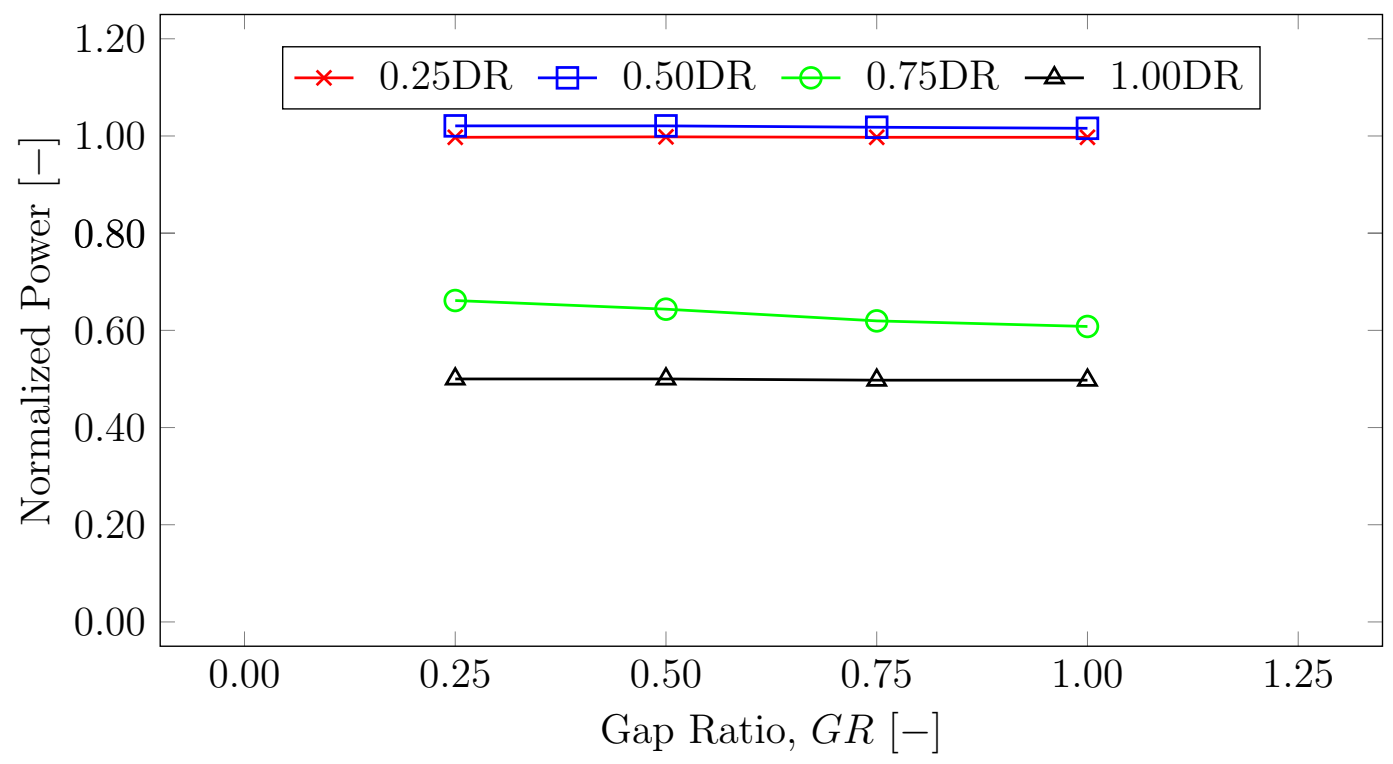

Figure 5.13: Power output comparison between four DRWT having various diameter ratios $(\mathrm{DR}=0.25,0.50,0.75$, and 1.00) at a CTSR of 7.5

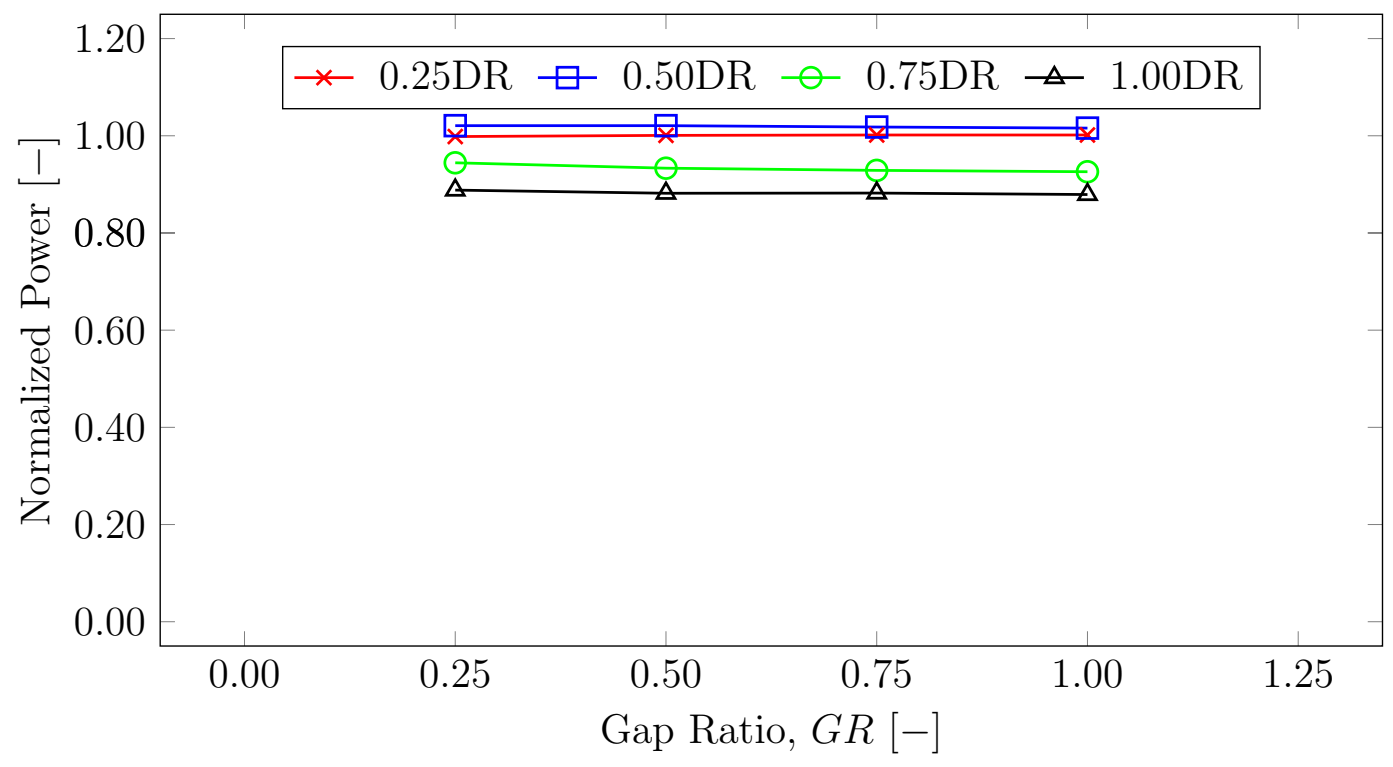

Figure 5.14: Power output comparison between four DRWT having various diameter ratios $(\mathrm{DR}=0.25,0.50,0.75$, and 1.00$)$ at $10 \mathrm{~m} / \mathrm{s}$ freestream velocity 


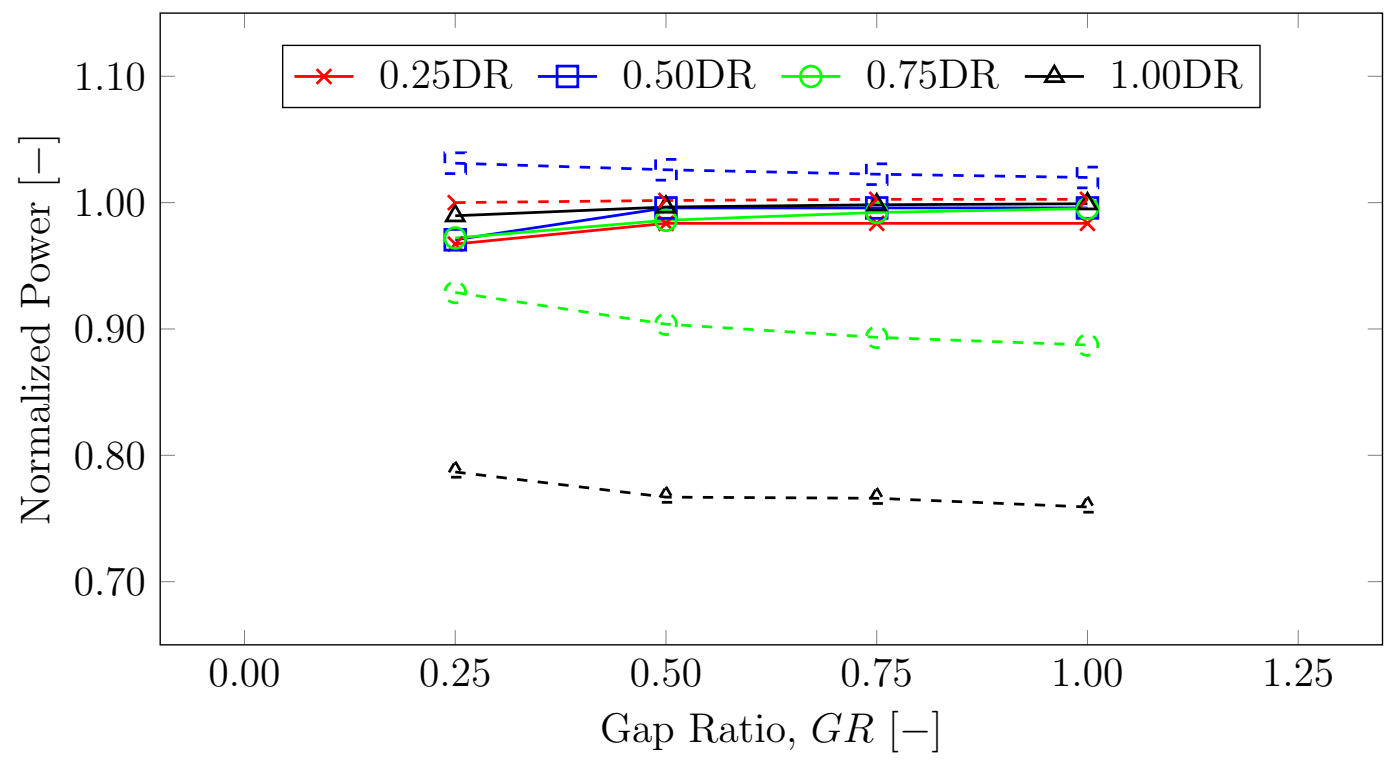

Figure 5.15: Performance of the upwind (solid lines) and downwind (dashed lines) rotors compared to their respective SRWT as a function of gap ratio at $10 \mathrm{~m} / \mathrm{s}$ freestream velocity

deficit of $21 \%$ obtained corresponds to the lower end of the range ( $25 \%-40 \%$ power loss from the downwind rotor) found in the literature $[5,6,16]$. It is believed that this lower deficit is due to the lack of tower effect in this study.

\subsubsection{CASE D04: Scaling Effect}

To verify the scalability of the three dimensionless parameters derived in this thesis, the performance of the baseline model was compared to two geometrically similar models scaled by a factor of 0.5 and 2. Figure 5.16 illustrates the baseline model along with the two scaled models. In other words, the three models investigated are equipped with upwind rotors having diameters of $2.5 \mathrm{~m}, 5.0 \mathrm{~m}$, and $10.0 \mathrm{~m}$ and downwind rotor diameters of $5.0 \mathrm{~m}, 10.0 \mathrm{~m}$, and $20.0 \mathrm{~m}$, respectively. Figure 5.17 shows the performance of the three geometrically-scaled dual-rotor models having 


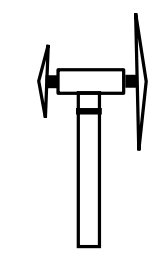

0.5 scale

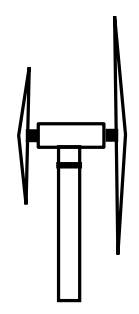

1.0 scale

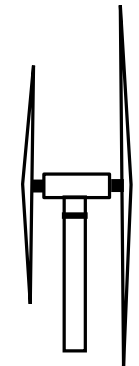

2.0 scale

Figure 5.16: Schematic of the three DRWT models investigated in Case D04

diameter and gap ratios of 0.50 . The large discrepancy which observed at 7.5 CTSR, can be justified by the fact that Reynolds number was not constant between the three models. Therefore, for the purpose of this study, Case D04 was repeated with the Reynolds number artificially kept constant by varying the fluid viscosity between the models. The results which are displayed using dashed lines in Figure 5.17, confirmed that the differences in power output between the models are solely due to Reynolds number effect.

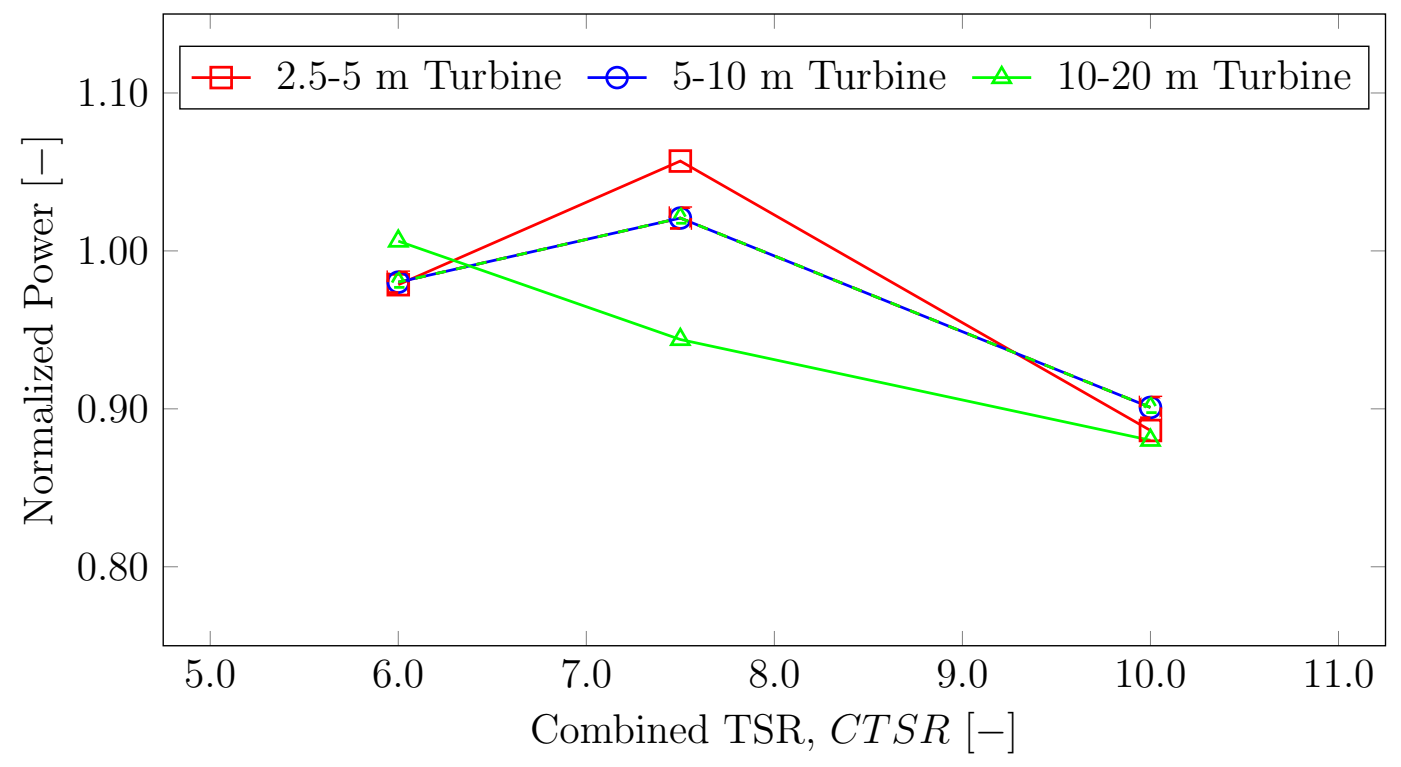

Figure 5.17: Power output comparison between three geometrically-scaled DRWT with diameter and gap ratios of 0.5 (Dashed lines represent the constant Reynolds number case) 


\section{Chapter 6}

\section{Conclusions and Recommendations for Future Work}

With dual-rotor wind turbines (DRWTs) attracting more attention in recent years, the present work aimed at developing a prediction tool and identifying potential dimensionless parameters for DRWTs as well as establishing an optimal DRWT configuration. This section is divided into two sub-sections summarizing (a) the work performed on the prediction tool including potential improvements and (b) the dimensionless parameters involved in the dual-rotor study.

\subsection{Prediction Tool}

An in-house vortex filament code, named DR_HAWT (Dual-Rotor Horizontal-Axis Wind Turbine code) was implemented in FORTRAN 95 for the aerodynamic performance prediction of a single or dual-rotor horizontal-axis wind turbine (HAWT). This three-dimensional (3D) free vortex model which is based on the lifting line theory, represents a wind turbine blade and its wake using straight vortex filaments. The performance prediction of DR_HAWT was shown to agree well with the single rotor results from two well-known wind tunnel experiments, namely the NREL Phase VI 
and the MEXICO wind turbines. However, in deep-stall condition, the accuracy of the prediction, after correcting for rotational effects, is poor which corresponds to the results found in the literature. Due to a lack of available DRWT experimental data, the vortex code was assumed validated after further investigations using various blade-vortex interaction scenarios.

From the current study, several additions can be included in DR_HAWT to improve its accuracy and computational cost. These improvements are listed below:

- Although parallel computing capabilities have already been implemented in DR_HAWT, the author believes that further parallelization of the vortex code is possible which will further reduce the computational cost. Moreover, in the current code, the effect of every vortex filaments is evaluated. This tedious task can be trimmed by either eliminating weaker vortices, or combining the nearby vortices into a stronger one, thereby reducing computational cost.

- Most single rotor wind turbine simulations are modeled without their tower, however, for dual-rotor configurations, the latter will significantly influence the performance of the downwind rotor. The effect of the tower may be aerodynamically modeled using semi-empirical tower shadow wake models such as Powles', Blevins', Schlichtings' and the JET wake models. These steady wake models describe the velocity deficit behind a body as a decaying Gaussian or cosine square shaped profile [107].

- Since wind turbines do not always experience head on winds, an additional input parameter such as yaw angle, can be included in the present version of DR_HAWT. This parameter, which describes the direction of the wind, can therefore allow for yaw flow conditions to be investigated. In addition, the yaw flow measurements from the NREL Phase VI and MEXICO experiments can 
be used to validate the single-rotor portion of the vortex code.

- Although the vortex code was compared to single-rotor experiments and showed good performance prediction, it is important to validate dual-rotor cases with in-house wind tunnel experiments employing either a dual-rotor setup or two single-rotors co-axially positioned. Based on the author's experience, XFOIL does not generate good aerodynamic polars at very low Reynolds number and therefore, $2 \mathrm{D}$ wind tunnel experiments may be required to obtain the necessary polar database to input in DR_HAWT.

- In this vortex code, a viscous core model incorporating the Biot-Savart law, is employed to simulate viscous diffusion of the vortex filaments based on the vortex age. However, vortex filaments also experience positive or negative strain (stretching or squeezing, respectively) in unsteady flow fields. This effect will cause a change in the local induced velocity field, which will influence the motion of the vortex filaments relative to one other [84]. Although viscous diffusion may be sufficient for single-rotor simulations, better DRWT performance prediction may be obtained if the downwind rotor sees a more accurate representation of the upwind wake.

\subsection{Dual-Rotor Study}

Three non-dimensional parameters, namely diameter ratio $\left(D_{1} / D_{2}\right)$, gap ratio $\left(L / D_{2}\right)$, and combined tip-speed ratio $\left(\omega_{1} R_{2} / V_{o}\right)$, were derived for the characterization of DRWTs. With the assumption that the blade tip-speed is conserved, 16 hypothetical DRWT models were created based on blade geometry and operating conditions of the NREL Phase VI wind turbine. For the purpose of the present work, uncorrected 2D aerodynamic polars obtained from XFOIL were employed to remove 
additional unknowns from the study since stall delay models are based on empirical work. Through three case studies, the effect of each parameter was analyzed using DR_HAWT. The following are observations and conclusions gathered from this study:

- Effect of Diameter Ratio: Diameter ratio plays a significant role in the power extraction of the downwind rotor. The addition of a smaller auxiliary rotor placed upstream of the main rotor allows for further kinetic energy extraction from the wind. However, as the swept area of the upwind rotor increases, the performance of the downwind rotor is affected as the latter is overshadowed by the former. It was also found that for a particular situation where a 0.5 diameter ratio turbine experienced an oncoming wind velocity of $10 \mathrm{~m} / \mathrm{s}$, more energy than that of its equivalent SRWTs was extracted since the operating range of the turbine shifted from deep-stall to stall condition.

- Effect of Gap Ratio: Due to a lack of wake expansion from the NREL Phase VI rotor, hardly any differences were obtained when analyzing the effect of gap ratio. However, if the effect of the tower and nacelle was also modeled, it is expected that performance of the downwind rotor will decrease for the case where the tower is located in-between the rotors.

- Effect of Combined Tip-Speed Ratio: The combined tip-speed ratio (CTSR) uses the characteristic of both the upwind and downwind rotors as well as the oncoming wind velocity. The present work showed that this dimensionless parameter can be used to compare the power generation of various dual-rotor systems with different diameter and gap ratios. Moreover, it is believed that the combined TSR will provide an insight on the performance of various DRWTs during the initial design stages when large number of configurations are being studied. 
- The results from the current work suggest that having a counter-rotating $5.0 \mathrm{~m}$ diameter auxiliary rotor located $5.0 \mathrm{~m}$ upstream of the $10 \mathrm{~m}$ diameter NREL Phase VI rotor could provide an optimal DRWT configuration. The overall trend suggests that the performance of DRWTs is enhanced at low diameter and gap ratios, keeping in mind that the minimum gap ratio allowed by a specific turbine is dependent on the mechanical system used. From this study, such optimal DRWT model could produce more than $90 \%$ of the power generated by equivalent SRWTs at an oncoming wind speed of $7.5 \mathrm{~m} / \mathrm{s}$.

- The addition of either a half-sized or full-sized rotor upstream of the NREL Phase VI rotor showed an increase in power extraction of up to about $25 \%$ and $76 \%$, respectively, compared to the power output of the downwind rotor only. This correlates well with the improved performance found in the literature (see Section 4.5).

From the current study, several paths can be followed to improve both the dimensionless parameters and in establishing an optimal DRWT configuration. The recommendations for future work are listed below:

- Although experimental work is associated to high cost, the effects of each dimensionless parameter can be analyzed through in-house wind tunnel experiments since the latter generally provide a more realistic representation. As previously mentioned, these measurements can also be used to further validate DR_HAWT.

- To better understand the effect of each dimensionless parameter on the performance of DRWTs, other wind turbine operating conditions and blade geometries should be investigated such as the three-bladed $4.5 \mathrm{~m}$ diameter MEXICO wind turbine. Moreover, since the blade geometry employed in the present work was 
designed to operate in a single-rotor configuration, a blade optimization can be performed for both the upwind and downwind rotors.

- The current study neglects the effect of the tower, however, since the latter will definitely influence the turbine's performance, it would be interesting to investigate whether the tower and its location with respect to the downwind rotor, affects the ability of the turbine to extract energy.

- Since dual-rotor system may be a potential solution to the increasing size of SRWT, it would be interesting to see how much larger a SRWT should be to extract similar power to a DRWT. In addition, further analyses should be performed under yaw conditions and flow misalignment to investigate if DRWT performs as well as SRWT under such conditions.

- In addition to the three dimensionless numbers found in this thesis, other numbers such as $L / D_{1}$ and $\omega_{2} R_{1} / V_{o}$, can be derived which could allow for better comparison between DRWT models operating under different Reynolds number. Hence, based on the results found in this study, further investigation into DRWT dimensionless parameters should be performed. 


\section{List of References}

[1] Canadian Wind Energy Association (CanWEA), "Ontario." http://canwea. ca/wind-energy/ontario/, 2016. Accessed: 01-12-2016.

[2] Canadian Wind Energy Association (CanWEA), "Wind Vision on 2025: Powering Canada's Future." http://canwea.ca/pdf/windvision/Windvision_ backgrounder_e.pdf, 2008. Accessed: 02-08-2015.

[3] Canadian Wind Energy Association (CanWEA), "Installed Capacity." http:// canwea.ca/wind-energy/installed-capacity/, December 2015. Accessed: 11-03-2016.

[4] V. Yaramasu, B. Wu, P. C. Sen, S. Kouro, and M. Narimani, "High-Power Wind Energy Conversion Systems: State-of-the-Art and Emerging Technologies," Proceedings of the IEEE, vol. 103, no. 5, pp. 740-788, 2015.

[5] R. W. Y. Habash, V. Groza, Y. Yang, C. Blouin, and P. Guillemette, "Performance of a Contrarotating Small Wind Energy Converter," ISRN Mechanical Engineering, pp. 1-10, 2011.

[6] A. Ozbay, W. Tian, and H. Hu, "An Experimental Investigation on the Wake Characteristics and Aeromechanics of Dual-Rotor Wind Turbines," ASME Journal of Engineering for Gas Turbines and Power, vol. 138, no. 4, p. 042602 (15 pages), 2016.

[7] IRENA, "Renewable Energy Technologies: Cosr Analysis Series," tech. rep., International Renewable Energy Agency (IRENA), 2012.

[8] R. L. Busby, Wind Power: The Industry Grows Up. PennWell Corporation, 2012.

[9] T. Burton, N. Jenkins, D. Sharpe, and E. Bossanyi, Wind Energy Handbook. John Wiley and Sons, 2011. 
[10] J. F. Manwell, J. G. McGowan, and A. L. Rogers, Wind Energy Explained Theory, Design and Application. John Wiley \& Sons, 2002.

[11] M. O. L. Hansen, Aerodynamics of Wind Turbines. Earthscan, 2008.

[12] J. G. Leishman, Aerodynamics of Horizontal Axis Wind Turbines, ch. 1, pp. 169. Springer-Verlag, 2011.

[13] S. Lee, H. Kima, E. Sona, and S. Lee, "Effects of Design Parameters on Aerodynamic Performance of a Counter-Rotating Wind Turbine," Renewable Energy, vol. 42, pp. 140-144, 2012.

[14] W. Yuan, W. Tian, A. Ozbay, and H. Hu, "An Experimental Study on the Effects of Relative Rotation Direction on the Wake Interferences among Tandem Wind Turbines," Science China Physics, Mechanics \& Astronomy, vol. 57, no. 5, pp. 935-949, 2014.

[15] S. N. Jung, T.-S. No, and K.-W. Ryu, "Aerodynamic Performance Prediction of a 30 kW Counter-Rotating Wind Turbine System," Renewable Energy, vol. 30, pp. 631-644, 2005.

[16] W. Z. Shen, V. A. K. Zakkam, J. N. Sørensen, and K. Appa, "Analysis of Counter-Rotating Wind Turbines," in Journal of Physics: Conference Series, vol. 75, 2007.

[17] A. Rosenberg, S. Selvaraj, and A. Sharma, "A Novel Dual-Rotor Turbine for Increased Wind Energy Capture," Journal of Physics: Conference Series, vol. 524, 2014.

[18] ZEC Wind Power Corp., "ZEC Wind Power." http://www.zecwindpower. com/, 2015. Accessed: 08/02/2016.

[19] P. Jamieson, Innovation in Wind Turbine Design. John Wiley \& Sons, Ltd, 2011.

[20] E. de Vries, "EXCLUSIVE: Vestas Tests Four-Rotor Concept Turbine." http://www.windpowermonthly.com/article/1391775/exclusive-vestastests-four-rotor-concept-turbine, April 2016.

[21] H. Snel, "Review of the Present Status of Rotor Aerodynamics," Wind Energy, vol. 1, pp. 46-69, 1998. 
[22] J. G. Leishman, "Challenges in Modelling the Unsteady Aerodynamics of Wind Turbines," Wind Energy, vol. 5, pp. 85-132, 2002.

[23] L. J. Vermeer, J. N. Sørensen, and A. Crespo, "Wind Turbine Wake Aerodynamics," Progress in Aerospace Sciences, vol. 39, pp. 467-510, 2003.

[24] M. O. L. Hansen, J. N. Sørensen, S. Voutsinas, N. Sørensen, and H. A. Madsen, "State of the Art in Wind Turbine Aerodynamics and Aeroelasticity," Progress in Aerospace Sciences, vol. 42, pp. 285-330, 2006.

[25] B. Sanderse, "Aerodynamics of wind turbine wakes," tech. rep., Energy Research Centre of the Netherlands, 2009.

[26] G. A. M. van Kuik, J. N. Sørensen, and V. L. Okulov, "Rotor Theories by Professor Joukowsky: Momentum Theories," Progress in Aerospace Sciences, vol. 73, pp. 1-18, 2015.

[27] V. L. Okulov, J. N. Sørensen, and D. H. Wood, "The Rotor Theories by Professor Joukowsky: Vortex Theories," Progress in Aerospace Sciences, vol. 73, pp. 19-46, 2015.

[28] D. Marten, J. Wendler, G. Pechlivanoglou, C. Nayeri, and C. O. Paschereit, "QBlade: An Open Source Tool for Design and Simulation of Horizontal and Vertical Axis Wind Turbines," International Journal of Emerging Technology and Advanced Engineering, vol. 3, no. 3, pp. 264-269, 2013.

[29] D. Marten, M. Lennie, G. Pechlivanoglou, C. N. Nayeri, and C. O. Paschereit, "Implementation, Optimization and Validation of a Nonlinear Lifting Line Free Vortex Wake Module within the Wind Turbine Simulation Code QBlade," in Proceedings of ASME Turbo Expo 2015: Turbine Technical Conference and Exposition, 2015.

[30] J. M. Jonkman, G. J. Hayman, B. J. Jonkman, and R. R. Damiani, "AeroDyn v15 Users Guide and Theory Manual," tech. rep., National Renewable Energy Laboratory (NREL), 2016.

[31] G. Hassan, "Bladed Theory Manual," tech. rep., Garrad Hassan \& Partners Ltd., 2013.

[32] A. van Garrel, "Development of a Wind Turbine Aerodynamics Simulation Module," tech. rep., Energy research Centre of the Netherlands (ECN), 2003. 
[33] F. Grasso, A. van Garrel, and J. G. Schepers, "Development and Validation of Generalized Lifting Line Based Code for Wind Turbine Aerodynamics," in 49th AIAA Aerospace Sciences Meeting, 30th ASME Wind Energy Symposium, (Orlando, FL., USA), 2011.

[34] T. Sant, Improving BEM-based Aerodynamic Models in Wind Turbine Design Codes. PhD thesis, University of Malta, 2007.

[35] M. H. M. Kloosterman, "Development of the Near Wake behind a Horizontal Axis Wind Turbine," Master's thesis, Delft University of Technology, 2009.

[36] T. Sebastian, The Aerodynamics and Near Wake of an Offshore Floating Horizontal Axis Wind Turbine. PhD thesis, University of Massachusetts Amherst, 2012 .

[37] H. Abedi, "Development of Vortex Filament Method for Aerodynamic Loads on Rotor Blades," Master's thesis, Chalmers University of Technology, Gothenburg, Sweden, 2013.

[38] A. Rosenberg and A. Sharma, "A Prescribed-Wake Vortex Line Method for Aerodynamic Analysis and Optimization of Multi-Rotor Wind Turbines," in North American Wind Energy Academy (NAWEA) Symposiom, 2015.

[39] S. G. Voutsinas, M. A. Belessis, and S. Huberson, "Dynamic Inflow Effects and Vortex Particle Methods," in European Wind Energy Conference Proccedings, pp. 428-431, 1993.

[40] F. N. Coton, T. Wang, and R. A. M. Galbraith, "An Examination of Key Aerodynamic Modelling Issues Raised by the NREL Blind Comparison," Wind Energy, vol. 5, pp. 199-212, 2002.

[41] J. Strickland, B. Webster, and T. Nguyen, "A Vortex Model of the Darrius Turbine: An Analytical and Experimental Study," tech. rep., Sandia National Laboratories, 1980.

[42] J. A. Michelsen, "Basis3D - A Platform for Development of Multiblock PDE Solvers," tech. rep., Technical University of Denmark, 1992.

[43] J. A. Michelsen, "Block Structured Multigrid Solution of 2D and 3D Elliptic PDE's," tech. rep., Technical University of Denmark, 1994. 
[44] N. N. Sørensen, "General Purpose Flow Solver Applied to Flow over Hills," tech. rep., Ris $\varnothing$ National Laboratory, 1995.

[45] D.-P. Molenaar, Cost-Effective Design and Operation of Variable Speed Wind Turbines. DUP Science, 2003.

[46] J. G. Schepers, A. J. Brand, A. Bruining, J. M. R. Graham, M. M. Hand, D. G. Infield, H. A. Madsen, T. Maeda, J. H. Paynter, R. van Rooij, Y. Shimizu, D. A. Simms, and N. Stefanatos, "Final Report of IEA Annex XVIII: Enhanced Field Rotor Aerodynamics Database," tech. rep., The Energy Research Center of the Netherlands (ECN), 2002.

[47] M. M. Hand, D. A. Simms, L. J. Fingersh, D. W. Jager, J. R. Cotrell, S. Schreck, and S. M. Larwood, "Unsteady Aerodynamics Experiment Phase VI: Wind Tunnel Test Configurations and Available Data Campaigns," tech. rep., National Renewable Energy Laboratory (NREL), Golden, Colorado, 2001.

[48] D. A. Simms, S. Schreck, M. M. Hand, and L. J. Fingersh, "NREL Unsteady Aerodynamics Experiment in the NASA-Ames Wind Tunnel: A Comparison of Predictions to Measurements," tech. rep., National Renewable Energy Laboratory (NREL), Golden, Colorado, 2001.

[49] S. Schreck, "The NREL Full-Scale Wind Tunnel Experiment: Introduction to the Special Issue," Wind Energy, vol. 5, pp. 77-84, 2002.

[50] J. G. Schepers and H. Snel, "Model Experiments in Controlled Conditions," tech. rep., The Energy Research Center of the Netherlands (ECN), The Netherlands, 2007.

[51] J. G. Schepers, K. Boorsma, T. Cho, S. Gomez-Iradi, P. Schaffarczyk, A. Jeromin, W. Z. Shen, T. Lutz, K. Meister, B. Stoevesandt, S. Schreck, D. Micallef, R. Pereira, T. Sant, H. A. Madsen, and N. Sørensen, "Final Report of IEA Task 29, Mexnext (Phase 1): Analysis of Mexico Wind Tunnel Measurements," tech. rep., The Energy Research Center of the Netherlands (ECN), 2012 .

[52] S. Schreck, "NREL UAE Sequence S Data," 2015. via private communications.

[53] K. Boorsma and J. G. Schepers, "Description of Experimental Setup MEXICO Measurements," Tech. Rep. ECN-X-09-0XX, The Energy Research Center of the Netherlands (ECN), 2009. 
[54] J. G. Schepers, K. Boorsma, S. Gomez-Iradi, P. Schaffarczyk, H. A. Madsen, N. Sørensen, W. Z. Shen, T. Lutz, C. Schulz, I. Herraez, and S. Schreck, "Final Report of IEA Wind Task 29: Mexnext (Phase 2)," tech. rep., The Energy Research Center of the Netherlands (ECN), 2014.

[55] J. G. Schepers, "MEXICO Wind Turbine Data," 2015. via private communications.

[56] H. Glauert, Aerodynamic Theory: A General Review of Progress Under a Grant of the Guggenheim Fund for the Promotion of Aeronautics, ch. Airplane Propellers, pp. 169-360. Berlin, Heidelberg: Springer Berlin Heidelberg, 1935.

[57] J. G. Leishman, Principles of Helicopter Aerodynamics. Cambridge University Press, 2000.

[58] A. C. Hansen and C. P. Butterfield, "Aerodynamics of horizontal-axis wind turbines," Annual Review of Fluid Mechanics, vol. 25, pp. 115-149, 1993.

[59] R. Williams, "Prediction and Assessment of the Surface-based Aeroacoustics of Vertical-axis Wind Turbines," Master's thesis, Carleton University, 2014.

[60] H. A. Madsen and F. Rasmussen, "A Near Wake Model for Trailing Vorticity Compared with the Blade Element Momentum Theory," Wind Energy, vol. 7, pp. 325-341, 2004.

[61] M. J. Stock, "Summary of Vortex Methods Literature (A living document rife with opinion)," 2007.

[62] F. M. Menter, "Zonal Two equation k- $\omega$ Turbulence models for aerodynamic flow," in AIAA 24th Fluid Dynamics Conference, 1993.

[63] P. Spalart and S. Allmaras, "A One-Equation Turbulence Model for Aerodynamic Flows," in AIAA 30th Aerospace Sciences Meeting and Exhibit, 1992.

[64] B. S. Baldwin and T. J. Barth, "A One-Equation Turbulence Transport Model for High Reynolds Number Wall-Bounded Flows," in AIAA 25th Aerospace Sciences Meeting, 1987.

[65] P. Spalart, "Strategies for turbulence modelling and simulations," International Journal of Heat and Fluid Flow, vol. 21, pp. 252-263, 2000. 
[66] S. Gupta and J. G. Leishman, "Comparison of Momentum and Vortex Methods for the Aerodynamic Analysis of Wind Turbines," in 43rd AIAA Aerospace Sciences Meeting and Exhibit, 2005.

[67] N. N. Sørensen, J. A. Michelsen, and S. Schreck, "Navier-Stokes Predictions of the NREL Phase VI Rotor in the NASA Ames $80 \mathrm{ft} \times 120 \mathrm{ft}$ Wind Tunnel," Wind Energy, vol. 5, pp. 151-169, 2002.

[68] A. Fereidooni, "Numerical Study of Aeroelastic Behaviour of a Troposkien Shape Vertical Axis Wind Turbine," Master's thesis, Carleton University, 2013.

[69] P. G. Saffman, Vortex Dynamics. Cambridge University Press, 1992.

[70] J. Katz and A. Plotkin, Low-Speed Aerodynamics. Cambridge Aerospace Series, 2nd ed., 2001.

[71] M. Drela, "XFOIL - An analysis and design system for low Reynolds number airfoils," in Low Reynolds number aerodynamics, vol. 54, pp. 1-12, Springer Berlin Heidelberg, 1989.

[72] M. D. Maughmer and J. G. Coder, "Comparisons of Theoretical Methods for Predicting Airfoil Aerodynamic Characteristics," tech. rep., U.S. Army Research, Development and Engineering Command, 2010.

[73] W. Z. Shen, W. J. Zhu, A. Fischer, N. R. Garcia, J. T. Cheng, J. Chen, and J. Madsen, "Validation of the CQU-DTU-LN1 series of airfoils," The Science of Making Torque from Wind, 2012.

[74] D. Ragni, C. S. Ferreira, and G. Correale, "Experimental investigation of an optimized airfoil for vertical-axis wind turbines," Wind Energy, 2014.

[75] J. J. Bertin and M. L. Smith, Aerodynamics for Engineers. Prentice Hall, 1998.

[76] W. R. M. Van Hoydonck, M. I. Gerritsma, and M. J. L. van Tooren, "On Core and Curvature Corrections used in Straight-Line Vortex Filament Methods," Journal of the American Helicopter Society, 2012.

[77] W. F. Phillips and D. O. Snyder, "Modern Adaptation of Prandtl's Classic Lifting-Line Theory," Journal of Aircraft, 2000.

[78] J. G. Leishman, M. J. Bhagwat, and A. Bagai, "Free-Vortex Filament Methods for the Analysis of Helicopter Rotor Wakes," Journal of Aircraft, 2002. 
[79] M. J. Bhagwat and J. G. Leishman, "Generalized Viscous Vortex Model for Application to Free-Vortex Wake and Aeroacoustic Calculations," in 58th Annual Forum and Technology Display of the American Helicopter Society International, 2002.

[80] M. J. Bhagwat and J. G. Leishman, "Self-Induced Velocity of a Vortex Ring Using Straight-Line Segmentation," Journal of the American Helicopter Society, vol. 59, 2014.

[81] H. Lamb, Hydrodynamics. Cambridge: Cambridge University Press, 6th ed., 1932.

[82] M. P. Scully, Computation of Helicopter Rotor Wake Geometry and its Influence on Rotor Harmonic Airloads. PhD thesis, Massachusetts Institute of Technology, 1975.

[83] G. H. Vatistas, V. Kozel, and W. C. Mih, "A Simpler Model for Concentrated Vortices," Experiments in Fluids, vol. 11, pp. 73 - 76, 1991.

[84] S. Ananthan and J. G. Leishman, "Role of Filament Strain in the Free-Vortex Modeling of Rotor Wakes," Journal of the American Helicopter Society, vol. 49, pp. 176-191, 2004.

[85] M. J. Bhagwat and J. G. Leishman, "Measurements of Bound and Wake Circulation on a Helicopter Rotor," Journal of Aircraft, vol. 37, no. 2, pp. 227-234, 2000 .

[86] H. Himmelskamp, Profile Investigations on a Rotating Airscrew. Reports and Translations / MAP Völenrode, 1947.

[87] H. Snel, R. Houwink, G. van Bussel, and A. Bruining, "Sectional Prediction of 3D Effects for Stalled Flow on Rotating Blades and Comparison with Measurements," in Proceedings of the European Community Wind Energy Conference, 1993.

[88] Z. Du and M. S. Selig, "A 3-D Stall Delay Model for Horizontal Axis Wind Turbine Performance Prediction," American Institute of Aeronautics and Astronautics, Inc. (AIAA), vol. 21, pp. 9-19, 1997.

[89] P. K. Chaviaropoulos and M. O. L. Hansen, "Investigating Three-Dimensional and Rotational Effects on Wind Turbine Blades by Means of a Quasi-3D NavierStokes Solver," Journal of Fluids Engineering, vol. 122, pp. 330-336, 2000. 
[90] C. Lindenburg, "Modelling of Rotational Augmentation Based on Engineering Considerations and Measurements," in European Wind Energy Conference (EWEC), 2004.

[91] C. Bak, J. Johansen, and P. B. Andersen, "Three-Dimensional Corrections of Airfoil Characteristics Based on Pressure Distributions," in European Wind Energy Conference \& Exhibition (EWEC), 2006.

[92] S. P. Breton, F. N. Coton, and G. Moe, "A Study on Rotational Effects and Different Stall Delay Models using a Prescribed Wake Vortex Scheme and NREL Phase VI Experiment Data," Wind Energy, vol. 11, pp. 459-482, 2008.

[93] C. Lindenburg, "Investigation into Rotor Blade Aerodynamics," tech. rep., Energy research Centre of the Netherlands (ECN), 2003.

[94] J. Johansen and N. N. Sørensen, "Aerofoil Characteristics from 3D CFD Rotor Computations," Wind Energy, vol. 7, pp. 283-294, 2004.

[95] J. L. Dowler and S. Schmitz, "A Solution-Based Stall Delay Model for Horizontal-Axis Wind Turbines," Wing Energy, vol. 18, pp. 1793-1813, 2015.

[96] R. P. J. O. M. van Rooij, A. Bruining, and J. G. Schepers, "Validation of Some Rotor Stall Models by Analysis of the IEA Annex XVIII Field Data," in Proceedings of the European Wind Energy Conference Er Exhibition, 2003.

[97] R. B. dos Santos Pereira, "Validating the Beddoes-Leishman Dynamic Stall Model in the Horizontal Axis Wind Turbine Environment," Master's thesis, Lisbon's Instituto Superior Tcnico, 2010.

[98] U. Ayachit, The ParaView Guide: A Parallel Visualization Application. Kitware, Inc., 2015.

[99] P. Renzoni, Discrete Vortex Modeling of a Blade-Vortex Interaction. PhD thesis, Rensselaer Polytechnic Inst., Troy, NY, 1987.

[100] J. Straus, P. Renzoni, and R. E. Mayle, "Airfoil Pressure Measurements During a Blade Vortex Interaction and a Comparison with Theory," AIAA 26th Aerospace Sciences Meeting, vol. 28, no. 2, pp. 222-228, 1988.

[101] P. Renzoni and R. E. Mayle, "Incremental Force and Moment Coefficients for a Parallel Blade-Vortex Interaction," AIAA Journal, 1989. 
[102] T. Sant, G. Van Kuik, and G. J. W. Van Bussel, "Estimating the Angle of Attack from Blade Pressure Measurements on the NREL Phase VI Rotor using a Free Wake Vortex Model: Axial Conditions," Wind Energy, vol. 9, pp. 549577, 2006.

[103] R. P. J. O. M. van Rooij, W. A. Timmer, and A. Bruining, "Determination of the Local Inflow Angle on Rotating Blades," in World Wind Energy Conference and Exhibition, (Berlin), 2002.

[104] D. Maniaci, Wind Turbine Design using a Free-Wake Vortex Method with Winglet Application. PhD thesis, Pennsylvania State University, 2013.

[105] S. McTavish, Identification of Wind Turbine Testing Practices and Investigation of the Performance Benefits of Closely-Spaced Lateral Wind Farm Configurations. PhD thesis, Carleton University, 2013.

[106] K. Dykes, A. Platt, Y. Guo, A. Ning, R. King, T. Parsons, D. Petch, P. Veers, and B. Resor, "Effect of Tip-Speed Constraints on the Optimized Design of a Wind Turbine," tech. rep., National Renewable Energy Laboratory (NREL), 2014.

[107] M. Reiso, The Tower Shadow Effect in Downwind Wind Turbines. PhD thesis, Norwegian University of Science and Technology (NTNU), 2013.

[108] J. L. Tangler, "The Nebulous Art of Using Wind Tunnel Aerofoil Data for Predicting Rotor Performance," Wind Energy, vol. 5, pp. 245-257, 2002.

[109] S. Guntur, C. Bak, and N. N. Sørensen, "Analysis of 3D Stall Models for Wind Turbine Blades Using Data from the MEXICO Experiment," in 13th International Conference on Wind Engineering (ICWE), (Amsterdam), 2011. 


\section{Appendix A}

\section{Full-Cosine Discretization}

In order to enhance the accuracy at the root and tip regions of a blade where large gradient in the bound circulation are obtained, the blade is divided into elements using a full-cosine discretization method $[32,34,108]$. For a given number of discretized elements $n$, the blade span is divided into $n+1$ equi-angle increments as shown in Figure A.1. Equation A.1 defines the radial position $r$ for a given section $i$.

$$
r_{i}=\frac{R}{2}\left(1-\cos \gamma_{i}\right)
$$

where $R$ is the blade radius and $\gamma_{i}$ is incremented angle for section $i$ as defined in Figure A.1.

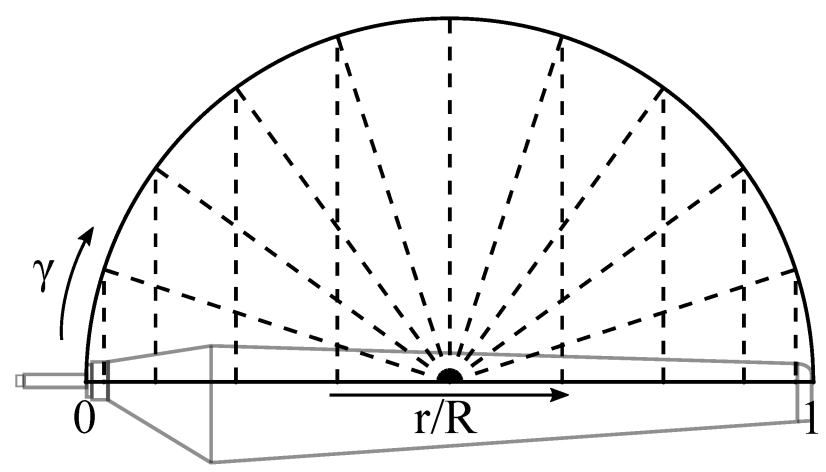

Figure A.1: Full-cosine discretization method applied on the NREL Phase VI 


\section{Appendix B}

\section{Inverse BEM}

The inverse BEM methodology which is adapted from the classical blade element momentum (BEM) theory, utilized the normal and tangential force coefficients obtained from experiments to evaluate the angle of attack, and the 3D lift and drag coefficients. The following describe the formulation used based on Refs. [93, 109]:

1. Initialize the axial $(a)$ and tangential $\left(a^{\prime}\right)$ induction factors, typically 0 or $1 / 3$.

2. Compute the local flow angle $\phi$ :

$$
\phi=\arctan \left[\frac{(1-a) V_{o}}{\left(1+a^{\prime}\right) \omega r}\right]
$$

3. Calculate the new induction factors using the experimental $C_{t}$ and $C_{n}$ :

$$
\begin{gathered}
a=\frac{1}{\frac{4 \sin ^{2} \phi}{\sigma C_{n}}+1} \\
a^{\prime}=\frac{1}{\frac{4 \sin \phi \cos \phi}{\sigma C_{t}}-1}
\end{gathered}
$$

4. If $a$ and $a^{\prime}$ has changed more than a certain tolerance, go to step 3 or else finish. 
5. Determine the local angle of attack $\alpha$ :

$$
\alpha=\phi-\beta-\theta_{p}
$$

6. Calculate the 3D lift and drag coefficients:

$$
\begin{aligned}
& C_{L, 3 D}=C_{n} \cos \phi+C_{t} \sin \phi \\
& C_{L, 3 D}=C_{n} \sin \phi-C_{t} \cos \phi
\end{aligned}
$$

It should be noted that Prandtl's tip and root losses as well as Glauert's correction for high induction factors can also be included. 